\title{
Generalization in the Presence of Free Variables: a Mechanically-Checked Correctness Proof for One Algorithm
}

\author{
Matt Kaufmann
}

Technical Report \#53

April, 1990

Computational Logic Inc.

1717 W. 6th St. Suite 290

Austin, Texas 78703

(512) 322-9951

This research was supported in part by ONR Contract N00014-88-C-0454. The views and conclusions contained in this document are those of the author and should not be interpreted as representing the official policies, either expressed or implied, of Computational Logic, Inc., the Office of Naval Research or the U.S. Government. 


\section{Introduction}

The motivation for this work began with a concern for the correctness of an implementation of logic. The system PC-NQTHM ${ }^{1}$ is an interactive " $p r o o f-c h e c k e r "$ enhancement of the Boyer-Moore Theorem Prover [3], and is documented in [10]. In [11] we report on an extension of this system that admits a notion of free variables. Roughly, free variables are ones that the user is allowed to instantiate in the course of a proof. An earlier version of this extension for free variables had a soundness bug in one of the commands, called GENERALIZE. (This command allows one to replace terms by new variables and proceed by proving the stronger, generalized version of the goal. Thus, it corresponds to the inference rule of universal instantiation). In fact the bug was easily corrected and the correctness of the resulting GENERALIZE command was checked on paper. However, the rude shock of having made a soundness mistake in the previous version led to the following goal: formalize the new version of the GENERALIZE command in the Boyer-Moore logic, and mechanically check a proof of correctness of this formalization.

In this paper we present a mechanically-checked proof of correctness for a generalization algorithm. Although the theorem itself is probably new (at least, we are unaware of any existing statement of it), the interest here lies not particularly in the theorem per se but, rather, lies in the demonstration of the use of mechanical verification for assisting in the reliability of detailed proofs and software. In particular, we believe that this exercise strongly suggests the feasibility of creating a verified version of PC-NQTHM, i.e. one which is proved correct in the Boyer-Moore theorem prover or in some successor of that system.

Thus, this paper could be viewed as a contribution to the study of metatheoretically extensible systems. Some reports of research in this spirit can be found in works of Davis and Schwartz [6], Weyhrauch [18], Boyer and Moore [2], Shankar [16], Knoblock and Constable [14, 13], Howe [9], and Quaife [15]. However, we also view this paper as an exposition which provides a rather detailed look at the practice of using the Boyer-Moore theorem prover and PC-NQTHM to proof-check mathematical arguments.

Although the development here is intended to capture the behavior of PC-NQTHM, it is actually an abstraction of that behavior. Hence, no familiarity with PC-NQTHM is required for an understanding of this document. Moreover, little particular understanding of the Boyer-Moore logic (cf. [1,3]) should be necessary for a comfortable reading of this paper (although for those interested, a complete treatment of the Boyer-Moore

\footnotetext{
1" $\mathrm{PC}$ ", for "proof-checker", "NQTHM" for the name commonly given to the current Boyer-Moore theorem prover
} 
theorem prover and the enhancements used here can be found in $[1,3,10,12,4,11])$. A summary of the basics needed in order to follow the treatment in this paper may be found in the first subsection 1.1 immediately below. We follow this with a very general discussion of the methodology employed in the use of the BoyerMoore theorem prover and PC-NQTHM in Subsection 1.2. A brief view of the main theorem and the high-level structure of its proof may be found in Subsection 1.3. We conclude this introduction with a summary of the remainder of the paper.

\subsection{Introduction to the Boyer-Moore logic and theorem prover}

For a description of the Boyer-Moore logic and theorem prover we refer the reader to the careful description in [3]. For now let us simply point out a few aspects of the logic and theorem prover.

One may simply view the Boyer-Moore logic as a version of first-order logic that has an induction rule of inference. Further details will be provided as needed during the presentation below. For now, let us simply note that a session with the Boyer-Moore theorem prover consists of a sequence of so-called events, which are generally either definitions or lemmas/theorems. A sequence of events stored at a given moment is called a history. Thus, this paper can be viewed as the presentation of a particular history that culminates in a lemma event stating the correctness of the algorithm in question.

There are a few built-in function symbols which, together with corresponding axioms, are part of the logic's basic (built-in) theory, i.e. are part of every history. Here is a summary of some of those that we will use in this paper. In each case we write terms in two ways. First, we write them in official s-expression (Lisp) notation, i.e. in the form ( $G t_{1} \ldots t_{n}$ ) where each $t_{i}$ is a term in that notation and $G$ is a function symbol (of the current history). Second, we write them in informal, more traditional notation. We will follow this convention throughout this paper. Moreover, we will write s-expressions using upper-case characters and traditional notation using lower-case characters. Here, then, are the primitives promised above.

- (CONS $\mathbf{X} \mathbf{Y}$ ) or $\langle\mathbf{x}, \mathbf{y}\rangle$ : the ordered pair formed from $\mathbf{x}$ and $\mathbf{y}$. CONS is also used to represent lists (sequences), in which case the atom NIL represents the empty list and (CONS $\mathbf{X} \quad \mathbf{Y}$ ) represents the sequence whose first element is $\mathbf{X}$ and whose remaining elements (in order) form the sequence $\mathbf{Y}$.

- (CAR z) or $\mathbf{1}^{\text {st }}(\mathbf{z})$ : the first component of the ordered pair $\mathbf{z}$

- (CDR $z)$ or $\mathbf{2}^{\text {nd }}(z)$ : the second component of the ordered pair $\mathbf{z}$

- (LISTP $\mathbf{z}$ ) or listp $(\mathbf{z}): \mathbf{z}$ is an ordered pair

- $\mathbf{T}$ : the boolean true

- $\mathbf{F}$ : the boolean false

- (LESSP $\mathbf{X} \mathbf{Y}$ ) or $\mathbf{x}<\mathbf{y}$ : $\mathbf{x}$ is less than $\mathbf{y}$ 
- (MEMBER $\mathbf{A} \mathbf{x}$ ) or $\mathbf{a} \in \mathbf{x}$ : $\mathbf{a}$ is a member of $\mathbf{x}$

The basic logic does not contain first-order quantification, so one often expresses quantified concepts using primitive recursion. Consider the following (irrelevant but instructive) definition of a predicate that holds of a list if and only if all of its elements are ordered pairs.

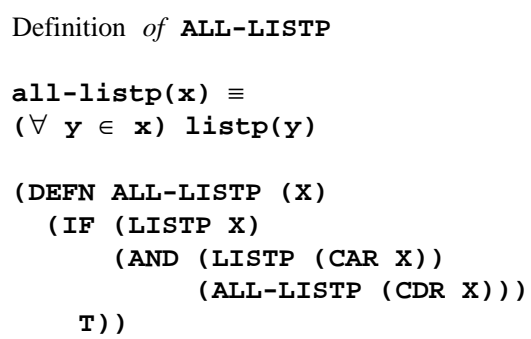

The first version of this definition is informal. In fact $\mathbf{x}$ is (presumably) a list, not a set (there is no built-in set type), so the predicate $\in$ doesn't really make precise sense here, though it's highly suggestive. We'll continue in this style throughout this paper.

The theorem prover contains a number of "processes", but most of the work is done by its simplifier, whose main component is a rewriter. The user labels certain lemmas as rewrite rules, and the system then rewrites using them. For example, consider the following rule, which says how the function ALL-LISTP above applies to a CoNS.

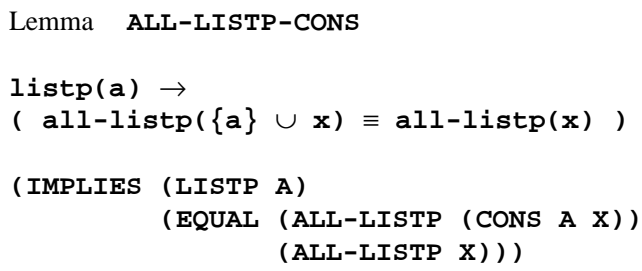

Again, the first version is merely suggestive, since the $\cup$ operator applies to sets, not lists. The name of this lemma is indicated to be ALL-LISTP-CONS. If we label it to be a rewrite rule then the theorem prover's rewriter will simplify any term of the form (ALL-LISTP (CONS $\mathbf{A} \mathbf{X}$ )) to the term (ALL-LISTP $\mathbf{X}$ ) provided it can establish (LISTP A). Again, while this extremely brief introduction to the logic and theorem prover should suffice as a prerequisite for the rest of the paper, the reader is welcome to consult [3] for a much more thorough treatment. 


\subsection{Remarks on methodology}

General hints on how to use the Boyer-Moore theorem prover may be found in the user's manual [3], particularly in Chapter 13. We also felt free to use PC-NQTHM, an interactive enhancement of the BoyerMoore theorem prover described in [10,11], to help explore some of the more difficult theorems. (Examples of such use may be found in [10].) However, the final proof script ultimately does not depend on PC-NQTHM, but only on the Boyer-Moore theorem prover with the enhancements for theories, LET, quantifiers, and functional variables mentioned above.

Our first completed proof was rather ugly ${ }^{2}$ in a number of places. Apparently this phenomenon is rather typical for users of the Boyer-Moore theorem prover, since one is still discovering the proper abstractions and proof structure while carrying out the proofs. In fact, the helpful output of the system can also distract one towards proving lemmas that are geared specifically to allow a particular proof attempt to succeed rather than towards proving elegant, general lemmas. Our first proof did, however, generate a number of basic definitions and rules for the files "sets.events", "alists.events", and "terms.events" which can be found (in their current forms) in the Appendix. So that we could obtain a proof script amenable to this exposition, we did the proof again, starting with those three files. Having those files already loaded allowed many of the proofs to go through automatically, which freed our attention for more substantive matters. In the course of the new proof a few additional basic rules were discovered and the three aforementioned libraries were suitably enhanced during this "polishing" process. Not surprisingly, when we moved some of those new basic rules up to those three files from our final file, some proofs in the final file no longer succeeded; when a rewrite rule is moved in front of a PROVE-LEMMA event, it can affect the course of the event's automatic proof. But we were able to find a few more useful rules for the three preliminary files, without undue difficulty. The resulting proof as it exists in the final file, "generalize.events", is reasonably concise. An advantage of this conciseness is that the result is quite amenable for description in the final two sections of this paper. Perhaps a disadvantage is that some of the struggles in completing the proof have been hidden, though we do make a few remarks about such difficulties where they came up.

We should be honest that although the lemmas stated in the final file "generalize.events" form the heart of the proof (in our view), still many of the supporting lemmas in the other three files are crucial too. A number of those lemmas were not only crucial to the main proof, but in fact were only discovered while trying to do that

\footnotetext{
${ }^{2}$ even compared to the final version!
} 
proof. ${ }^{3}$ The point here is that although the lemmas have been arranged into files for expository purposes, one should not be left with the impression that the first three files were created in isolation and then a fairly natural proof evolved without difficulty, as represented in "generalize.events". An unfortunate amount of sweat went into that proof! On the other hand, the original proof took well under a month, including the libraries and the time required to think about the theorem. So although our experience is that this kind of program verification remains a less-than-automatic activity, still we are not too disappointed by the amount of effort required. The exposition in this paper, however, is a different matter; it seemed quite time-consuming. We don't recommend such detailed expositions in general, although we hope that this one has pedagogical value.

We did not keep the set of lemmas in those first three files at a minimum. Rather, we were happy to build up less-than-minimal but useful libraries of rules. Therefore the thickness of the first three files in the Appendix is not entirely indicative of what is truly necessary for the successful processing of the events in the final file. On the other hand, we view the events in the first three files as being sufficiently fundamental that many or all of them should be usable in possible future work that involves notions such as lists, terms and substitutions.

Another obligation arising from honesty requires us to point out that hints to the Boyer-Moore prover have been omitted from the exposition below (although they do appear in the appendix). We simply felt that the hints would distract the reader from more substantive considerations, and would even be misleading in the absence of explanation.

Finally, let us remark that the time required to automatically replay the events constructed for this exercise was roughly an hour and a quarter on a Sun 3/60 with 20 megabytes main memory. Slightly under a half hour was spent on the events in the three preliminary files; the rest was spent on the events in "generalize.events".

\subsection{Outline of theorem and proof}

The main theorem is stated precisely in Section 4. However, here is a very informal version.

We want to model a proof development methodology similar to the one in PC-NQTHM [10,11], as explained at the start of the introduction. (In fact, similar "proof refinement" methodologies have been

\footnotetext{
${ }^{3}$ People familiar with the Boyer-Moore prover will correctly guess that many of these lemmas were thought up by reading failed proof transcripts and thinking about what might be useful to prove as rewrite rules. Others were discovered by crawling around through terms using PC-NQTHM.
} 
implemented in systems preceding PC-NQTHM as well, for example LCF [7] and its "descendents" HOL [8] and Nuprl [5].) In the PC-NQTHM methodology, the user starts with a proof state consisting of a single goal, namely the goal to be proved, and proceeds to create new proof states by "refining" goals into subgoals and simplified goals. The proof is complete when all goals of the state are simply $\mathbf{T}$ (true). Let us explain this more carefully (but still informally).

First imagine a situation where one has a formula in some logic that he wishes to show is a theorem. One approach would be to replace that formula with a list new formulas whose conjunction implies the given formula. (Such a step may be called a "refinement step".) The resulting formulas are then the goals that remain to be proved. The first formula in this list, which we will call the current (or top) goal, may then be similarly refined into subgoals that imply it, leaving one with those new goals, together with the existing goals other than that current goal. Once a current goal is simply the formula $T$ (true), it is replaced by the empty list of goals. One would hope to be able to continue this process until there are no goals left, in which case one can conclude that the original goal is a theorem. Such a sequence of steps will be called a "proof", though it is perhaps better viewed as a demonstration that a proof exists in that logic.

We might call the current list of (as yet unproved) goals the "current proof state". However, imagine a slightly more general paradigm in which a proof state consists not only of unproved goals but also of a list of variables called the free variables of that proof state. The idea is that one should be free to substitute for the free variables. For example, suppose there is a single goal, of the form $t_{1}<t_{2}$. Clearly it suffices to find some $\mathbf{z}$ for which $t_{1}<\mathbf{z}$ and $\mathbf{z}<t_{2}$. So, it should be legal to replace the current goal $t_{1}<t_{2}$ with a list of the two goals $t_{1}<\mathbf{z}$ and $\mathbf{z}<t_{2}$, with the stipulation that $\mathbf{z}$ is to be considered free. Then if we are able to find some term $\mathbf{u}$ for which we can prove $t_{1}<\mathbf{u}$ and $\mathbf{u}<t_{2}$, then we will be allowed to substitute $\mathbf{u}$ for $\mathbf{z}$ and carry out that proof.

Suppose a proof state has the property that there is some way of substituting terms for its free variables, into its goals, such that the resulting goals are all theorem. Such a proof state will be called valid. The "key lemma" for a proof of correctness of such a refinement-based system would establish that each refinement transformation has the following property: whenever the new state is "valid" in an appropriate sense, then the given state is "valid". For then an easy induction would let one conclude that if one performs a series of such state transformations, starting with the user's given goal and resulting in a state where all goals are disposed of, then (as such a final state is presumably "valid") the original state is "valid" -- and hence, presumably, the original goal is a theorem. 
Such a refinement system may have a number of legal refinement steps, so for a correctness result of the type described in the previous paragraph, one would have to prove a "key lemma" for each of these. We confine ourselves in this paper to such a proof for a single refinement step that we call generalization. The idea is that if one wishes to prove a goal $\mathbf{g}$ containing a subterm $\mathbf{t}$, it should be legal to replace $\boldsymbol{t}$ in $\mathbf{g}$ by a new variable. Standard logics have the property that if the result is a theorem, then the original goal is a theorem.

There is a subtlety which makes this correctness proof not completely trivial, namely, generalization in this sense is not sound in general, i.e. the aforementioned key lemma may fail to hold. The problem has to do with free variables, and examples are given in Subsection 4.1. Rather than get into details at this point, let us simply state that there is a way to define generalization so that it is correct and reasonable.

The main theorem in this paper states the correctness of a formalization of generalization in this context.

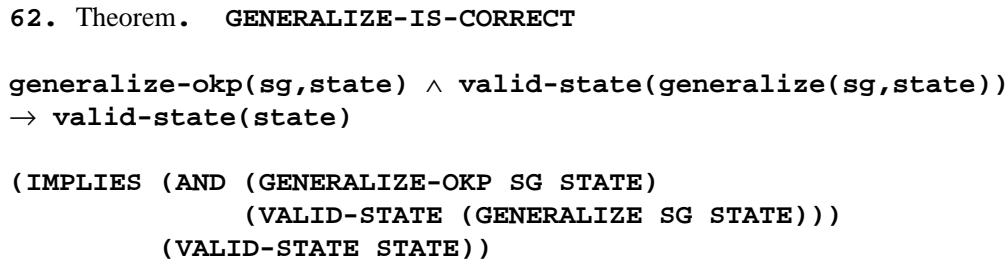

Here GENERALIZE-OKP is a predicate which may be viewed as a precondition under which the user is allowed to apply the GENERALIZE refinement rule. We also prove the much simpler "sanity" theorem, saying that if generalization is legal then the result is still a state. We'll say no more about this, except to mention that it could be useful in case we wish (someday) to extend the current theorem to handle a sequence of PC-NQTHM-like commands.

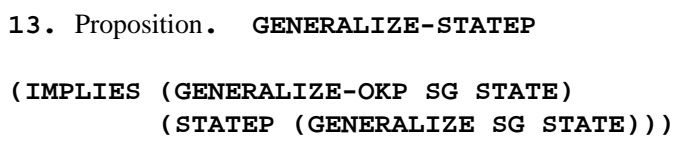

The function GENERALIZE is actually rather subtle, and the proof is more subtle than one might initially expect. Our approach in the mechanized proof-checking exercise was to break this theorem into major subtheorems, some of which were broken down further, and so on. In each case we checked that the theorem followed from its subtheorems, by adding the subtheorems as (temporary) axioms and running the Boyer-Moore theorem prover on the desired theorem (after proving minor subtheorems on which the theorem also depends; these are omitted in the diagram below). This approach will appear upon inspection of the file "generalize.events" in the Appendix. In fact this top-level structure of the proof is rather evident upon inspection of the final file "generalize.events" in the Appendix, and is also evident in the structure of the final 
section of the paper. Here is a brief summary, for convenience. We refer to the theorems by name as well as by the numbers associated with them in the file "generalize.events".

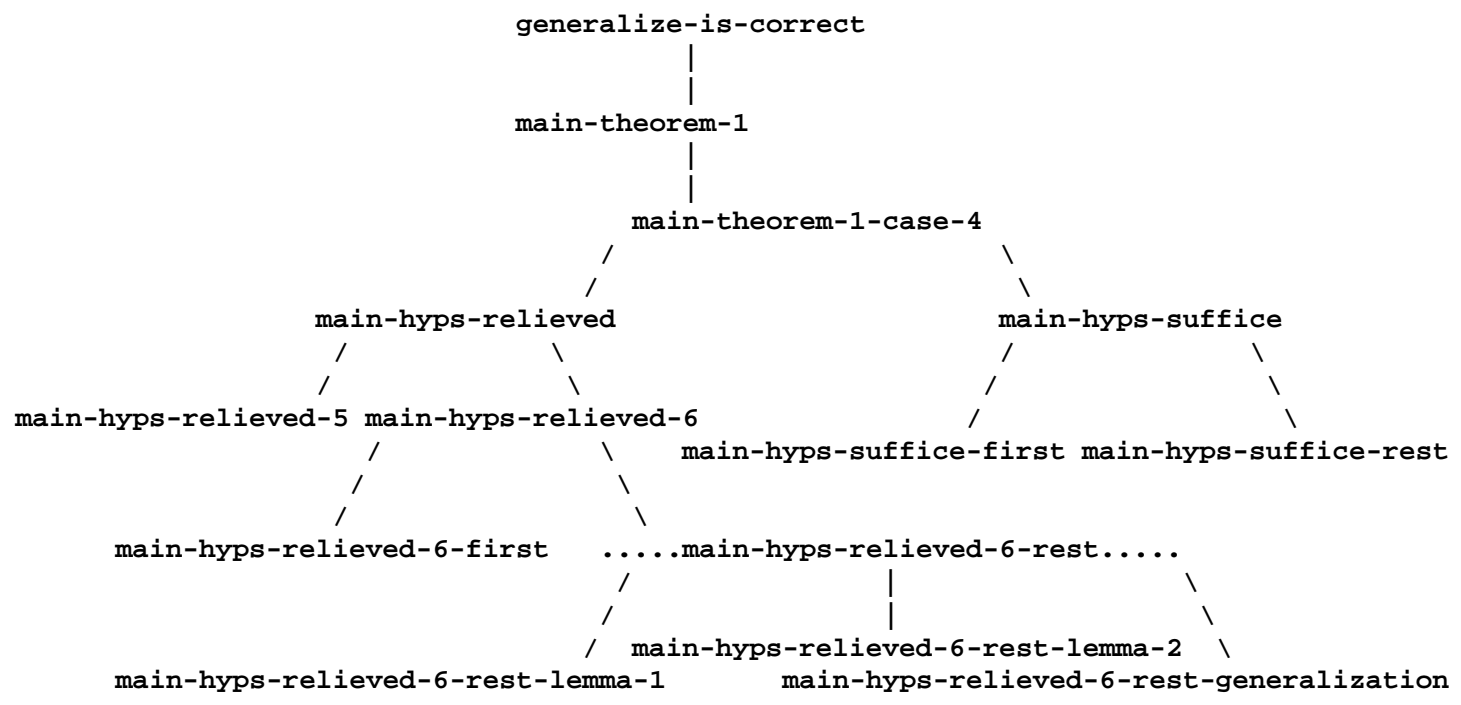

\subsection{Summary of the rest of the paper}

It's problematic how best to describe a proof checked with the Boyer-Moore prover. The appendix at the end of this report contains a complete list of events, including supporting events about sets, alists, terms, and proof theory. However, most readers will only find this list helpful for reference, at best. In the paper proper we outline a proof of the main theorem with a liberal amount of explanation. The development will refer to events in the appendix, but (as indicated above) will also display the events using conventional mathematical notation. Therefore, familiarity with Lisp notation is not a prerequisite for being able to follow the treatment here. There actually is one exception that we mention now: semicolons (;) denote the start of comments, so that all characters from a semicolon up to the end of the line should be viewed as informal comments only.

The following section (Section 2) presents the underlying logical preliminaries such as the notion of term. That is followed by a presentation in Section 3 of some basic but important lemmas about these notions. Section 4 then presents further notions specific to the theorem in question, culminating with a statement of that theorem. Finally, Section 5 contains a proof of the theorem that closely follows the mechanically-checked proof. Thus, one may view the final section either as being simply an informal proof of the theorem in English or as being a guide to the mechanically-checked proof.

The appendices contain four files of events that replay in the Boyer-Moore theorem prover as extended by notions of theories and LET notation (as described in [10]), first-order quantifiers (as described in [12]), and functional variables (as described in [4]). The first three of these sequences of events can be viewed as basic 
supporting libraries, corresponding to Sections 2 and 3 below. The last file may be viewed as the proof proper, including relevant definitions, and thus corresponds to the final two sections below.

\section{Basic Notions}

This section presents a number of primitive notions such as those of a variable, a term, and a substitution. Though these are quite standard, we state here the definitions of these notions used in the mechanically-checked proof development. We divide into subsections corresponding to the event files "sets.events", "alists.events", and "terms.events", all of which may be found in the Appendix, where complete definitions may be found. A brief introduction to the Boyer-Moore logic and to some of our conventions in this paper may be found in Subsection 1.1 above.

\subsection{Sets}

The event file "sets.events" forms the lowest-level foundation for our proof development. Here is a brief and very informal description of some of the functions defined in that file. The reader is referred to the Appendix for the actual definitions and for a number of basic lemmas. For convenience we indicate ordinary mathematical notation which "corresponds" to these notions. The correspondence isn't quite accurate since we will feel free to ignore the distinction between sets and lists for this purpose.

- (LENGTH L) or $|\mathbf{I}|$ : the number of elements in the list $\mathbf{L}$

- (SUBSETP $\mathbf{x} \mathbf{Y}$ ) or $\mathbf{x} \subseteq \mathbf{y}$ : equals $\mathbf{T}$ if every member of the list $\mathbf{x}$ is a member of the list $\mathbf{y}$, otherwise returns $\mathbf{F}$

- (DELETE $\mathbf{X}$ L) or $\mathbf{I} \backslash\{\mathbf{x}\}$ : the result of deleting the first occurrence of $\mathbf{X}$ from the list $\mathbf{L}$

- (DISJOINT $\mathbf{X} \mathbf{Y}$ ) or $\mathbf{x} \cap \mathbf{y}=\varnothing$ : equals $\mathbf{T}$ if $\mathbf{X}$ and $\mathbf{Y}$ share no common member, else $\mathbf{F}$

- (INTERSECTION $\mathbf{X} \mathbf{Y}$ ) or $\mathbf{x} \cap \mathbf{y}$ : the subsequence of the list $\mathbf{x}$ consisting of members of the list $\mathbf{y}$

- (SET-DIFF $\mathbf{X} \mathbf{Y}$ ) or $\mathbf{x} \backslash \mathbf{y}$ : the subsequence of the list $\mathbf{x}$ obtained by removing members of the list $\mathbf{y}$

- (SETP $\mathbf{X})$ or $\operatorname{set} \mathbf{p}(\mathbf{x})$ : equals $\mathbf{T}$ if the list $\mathbf{x}$ contains no duplicates, else $\mathbf{F}$

- (MAKE-SET $\mathbf{X}$ ) or make-set $(\mathbf{x})$ : a list with no duplicates that has the same members as does $\mathbf{x}$

\subsection{Alists}

Here is a brief and very informal description of some of the functions defined in the file "alists.events". The reader is referred to the Appendix for the actual definitions and for a number of lemmas.

- (ALISTP $\mathbf{X}$ ) or alistp (x): equals $\mathbf{T}$ if $\mathbf{x}$ is an association list (alist), i.e. a list of ordered pairs

- (DOMAIN MAP) or domain (map): a list of all first components of ordered pairs from map

- (RANGE MAP) or range (map) : a list of all second components of ordered pairs from MAP 
- (VALUE X MAP) or map (x): the second component of the first ordered pair in map whose first component is $\mathbf{x}$; we speak of this as being the value associated with $\mathbf{x}$ in map

- (INVERT MAP) or map ${ }^{-1}$ : returns the alist obtained by switching the first and second components of every ordered pair belonging to map.

- (MAPPING MAP) or mapping (map): equals $\mathbf{T}$ if map is an alist whose domain has no duplicates, else $\mathbf{F}$

- (RESTRICT S NEW-DOMAIN) Or $\mathbf{S}$ | new-domain: the subsequence of $\mathbf{s}$ consisting of pairs whose first components are members of new-domain.

- (CO-RESTRICT S NEW-DOMAIN) or S | new-domain: the subsequence of $\mathbf{s}$ consisting of pairs whose first components are not members of new-domain.

\subsection{Terms}

One typically defines the notion of term by recursion: a term is either a variable or the application of a function symbol to a list of terms (of an appropriate length). Our formal definitions of term and of various auxiliary notions will parallel this informal recursive one. This subsection is a summary of the file "terms.events", which may be found in the Appendix.

We'll begin with the notion of a variable. We could define the function VARIABLEP, thus specifying it as a unique function. However, we prefer to add an axiom asserting only some reasonable properties of this function, so as not to over-specify the notion of variable. Since the act of simply adding an axiom ${ }^{4}$ does not guarantee in general that the resulting theory is consistent, instead we will use an extension of the Boyer-Moore logic reported in [4] which allows an event form called CONSTRAIN. Perhaps the best way to explain CONSTRAIN is in the context of the example displayed below. The event below has name VARIABLEPINTRO, and the designation (REWRITE) indicates that it is to be stored as one or more rewrite rules. It asserts that no LISTP object (i.e. ordered pair) is a variable, and that VARIABLEP returns a boolean value. The last argument of CONSTRAIN below, namely ( (VARIABLEP NLISTP)), instructs the system to show that this axiom is consistent by showing that it holds when VARIABLEP is replaced by the function NLISTP (which is a predicate holding of objects that are not ordered pairs). Thus, we'll refer to this argument of a CONSTRAIN event as the witnessing alist. In fact, use of CONSTRAIN guarantees more than consistency -- it guarantees conservativity, in that no new theorems can be proved for the existing history in the presence of this axiom (see [4] for more on this).

\footnotetext{
${ }^{4}$ with the Boyer-Moore event type ADD-AXIOM
} 


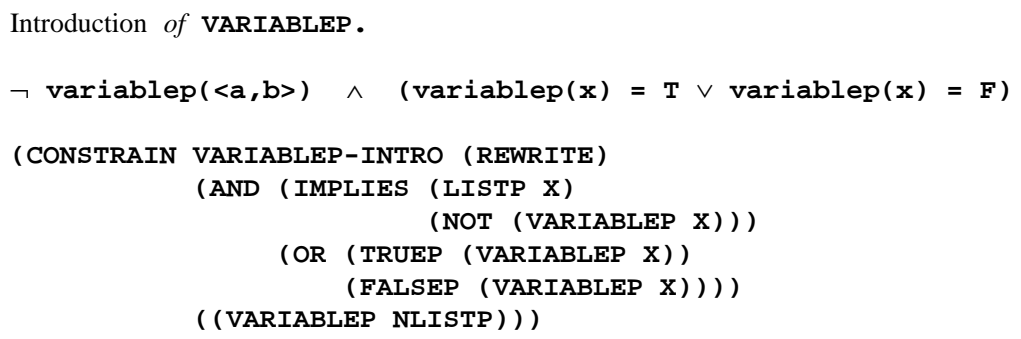

The function VARIABLE-LISTP recognizes lists of variables. We use the standard mechanism for representing quantification over lists in the Boyer-Moore logic, namely, primitive recursion.

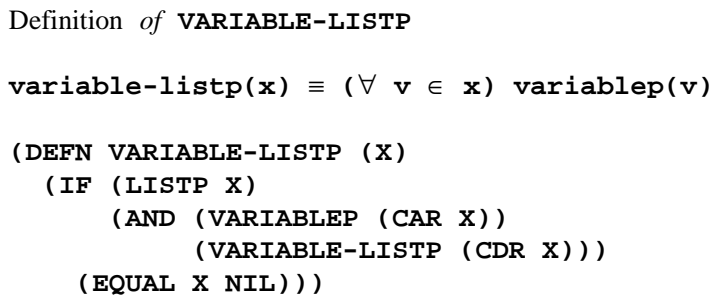

The next notion auxiliary to the notion of term is that of a function symbol. It is not important for the development that follows to know anything about the notion of a function symbol except that there is at least one 0-place function symbol (i. e. constant symbol), which we call (FN). Below is the appropriate CONSTRAIN event, which introduces FUNCTION-SYMBOLP and FN and asserts that (FN) is a function symbol. Notice that the "witnessing alist" suggests that the prover check this axiom with FUNCTION-SYMBOL-P replaced by LITATOM and with FN replaced by the constant function that returns the literal atom ' ZERO.

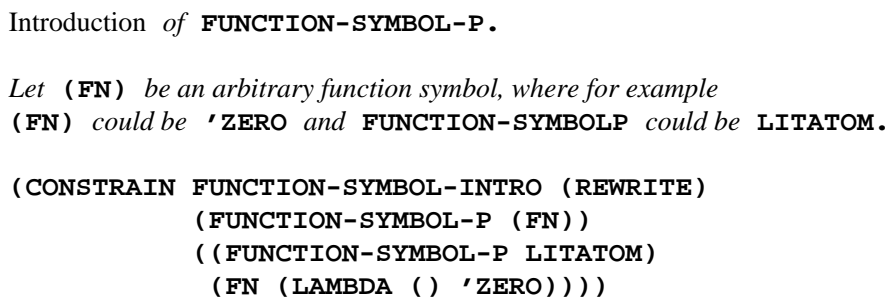

Now in order to define the notion of a term one has to define the notion of a list of terms as well. We will define these using mutual recursion, employing a standard trick for representing mutually recursive definitions in the Boyer-Moore logic: if FLG is not $\mathbf{F}$ then (TERMP $\mathbf{F L G ~} \mathbf{X}$ ) asserts that $\mathbf{X}$ is a term (informally, termp (x)), and otherwise (TERMP FLG $\mathbf{x}$ ) asserts that $\mathbf{x}$ is a list of terms (informally, termp-list $(\mathbf{x}))^{5}$

\footnotetext{
${ }^{5}$ Some Boyer-Moore prover users like to use 'LIST and $\mathrm{T}$ for the two explicitly-mentioned values of the FLG parameter in such situations. However, we found that a heuristic for defeating excessive backchaining was defeating some of our rewrite rules in that case.
} 


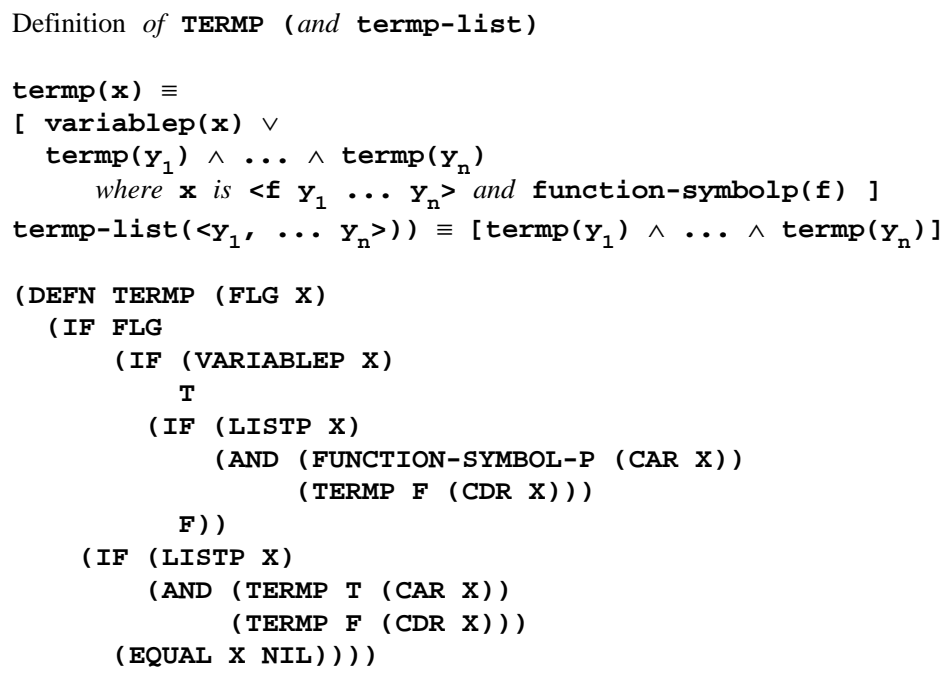

The function ALL-VARS returns a list of all variables in $\mathbf{x}$, where $\mathbf{x}$ is a term if $\mathbf{f l} \mathbf{g}$ is not $\mathbf{F}$ and a list of terms if $\mathbf{f l} \mathbf{g}$ is $\mathbf{F}$ ). It does not bother to eliminate duplicates.

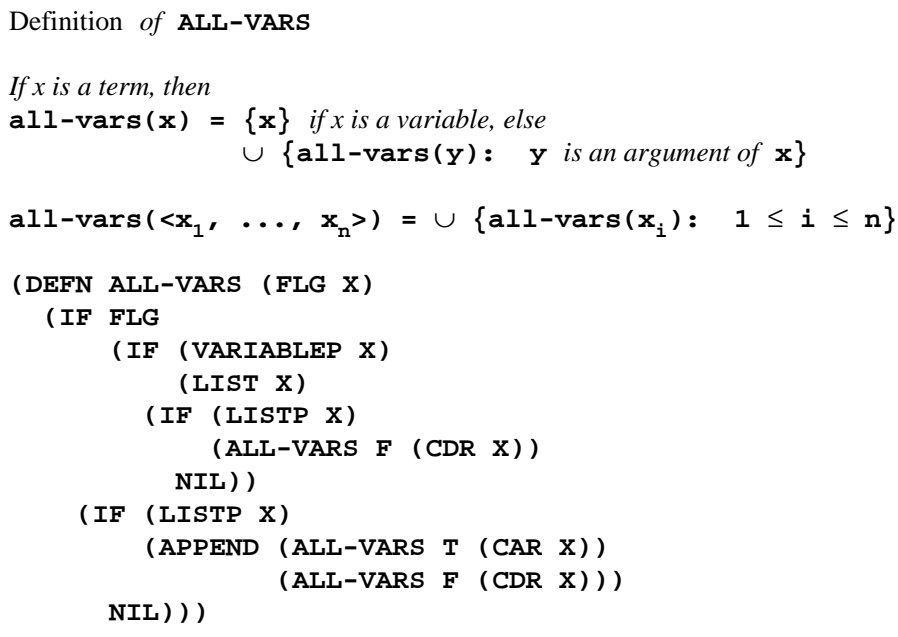

We also need to implement some notion of instantiation. A substitution is essentially a function that maps terms to terms, represented as a list of term pairs. Of particular interest is the class of variable substitutions, where the domain consists of variables:

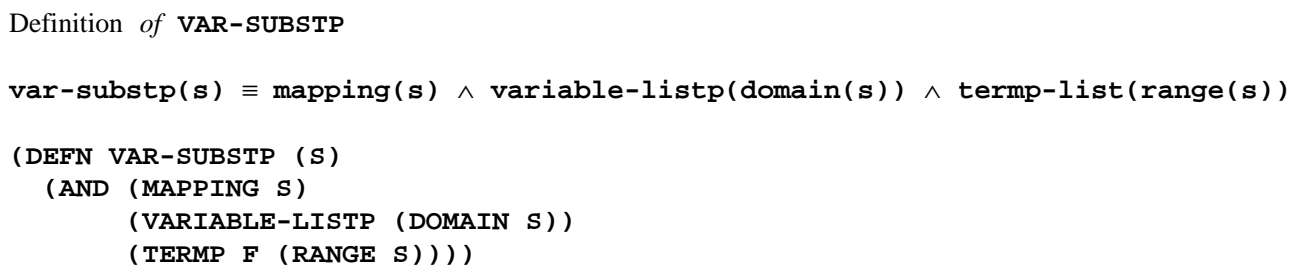

Given a substitution s (not necessarily a variable substitution), we define the instantiation 
of a term (or term list) $\mathbf{x}$ under the substitution $\mathbf{s}$ as follows. Notice that we follow the usual convention with respect to the parameter $\mathbf{f l g}$, namely if $\mathbf{f} \mathbf{l g}$ is $\mathbf{F}$ then $\mathbf{x}$ is a list of terms, and otherwise $\mathbf{x}$ is a single term.

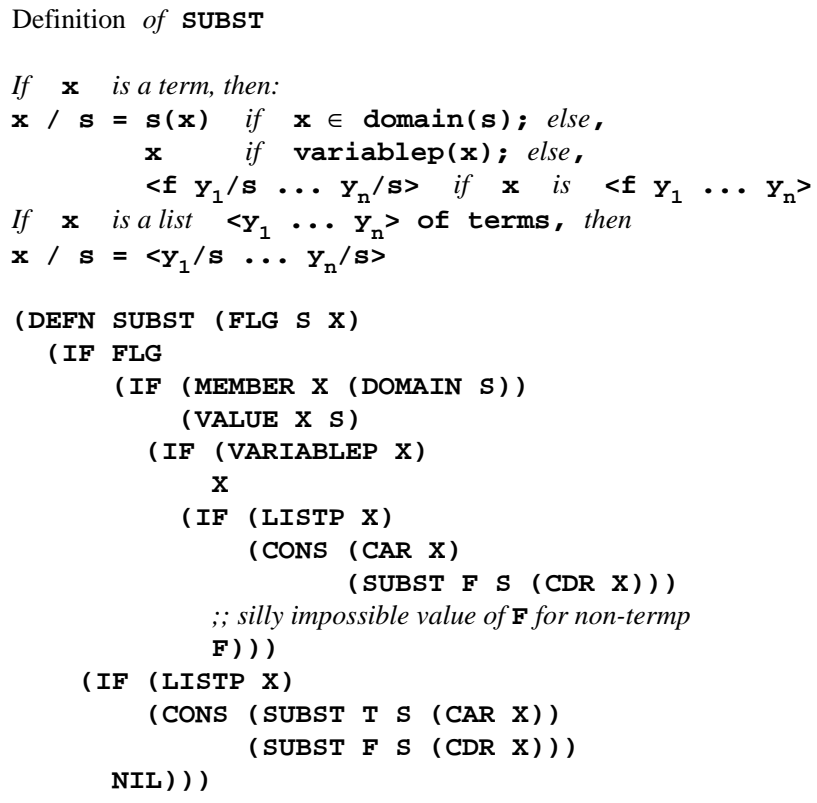

The following simple fact is one of many obvious facts that need to be proved. It says that the property of being a term (or term list, if FLG is $\mathbf{F}$ ) is preserved by the application of a substitution.

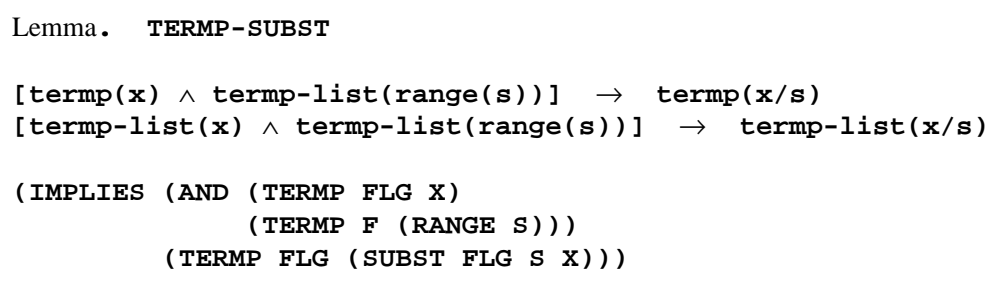

Just as SUBST is used to apply a substitution to a term, the function APPLY-TO-SUBST is used to apply one substitution to another substitution, i.e. to apply a substitution s1 to each term in range of another substitution $\mathbf{s} 2$. We may informally write

$$
\text { s2 // s1 }
$$

to denote the application of $\mathbf{s} \mathbf{1}$ to $\mathbf{s} \mathbf{2}$ in this sense. Formally, we have:

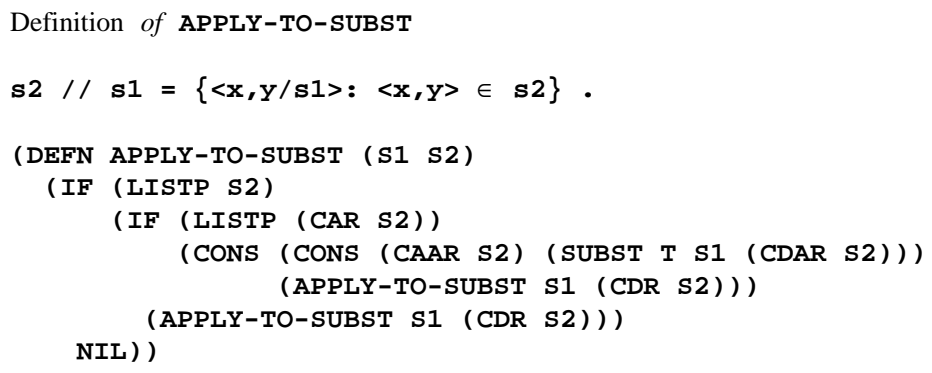


We may now define the composition of substitutions $\mathbf{s} \mathbf{1}$ and $\mathbf{s} \mathbf{2}$, which we write as (s1 • $\mathbf{s} \mathbf{2}$ ). This is the substitution that, when applied to a term, is the same as the result of first applying the substitution $\mathbf{s} 1$ and then the substitution $\mathbf{s} 2$. Let us display the definition of composition first in informal notation and then in formal notation. (Here is a minor detail for those familiar with the Boyer-Moore logic or Lisp: the definition of COMPOSE in the Boyer-Moore logic may safely use APPEND rather than UNION because the function VALUE only looks for the first occurrence of the key for which the value is to be found.)

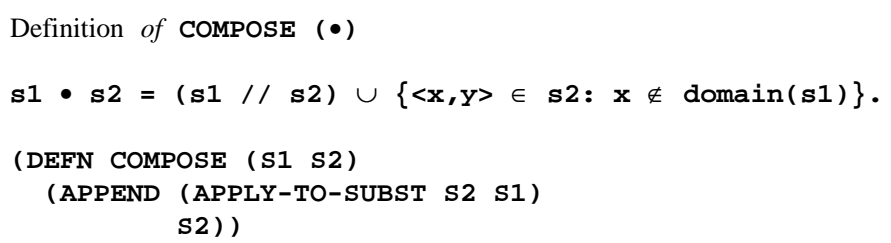

The following lemma shows that COMPOSE behaves similarly to ordinary function composition. We write the lemma both in informal and in formal notation.

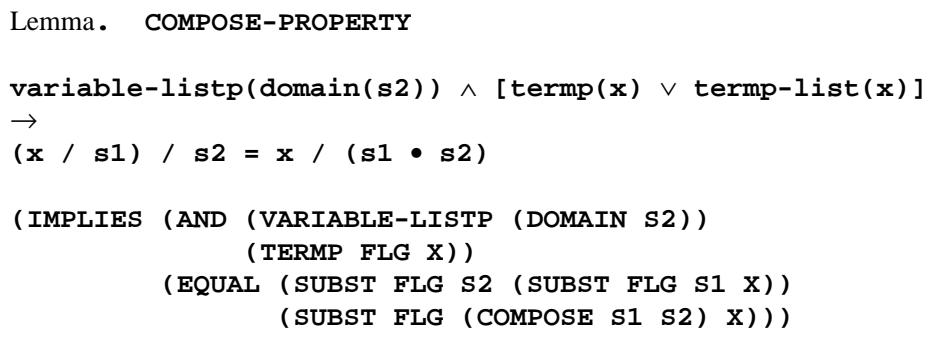

The next notion illustrates our first use of quantifiers in this development. An extension of the BoyerMoore logic and prover by first-order quantification is reported in [12]. Briefly, the idea is that there is a new event DEFN-SK, where the suffix "-SK" refers to Skolemization, a well-known means for removing quantifiers that was invented by the logician Thoralf Skolem. Every DEFN-SK event in fact adds quantifierfree axioms that uniquely define the indicated function symbol in a conservative extension (cf. 2.3) of the existing history. The DEFN-SK event below asserts that TERM1 is an instance of TERM2, with the usual convention that FLG indicates whether these are terms or term lists.

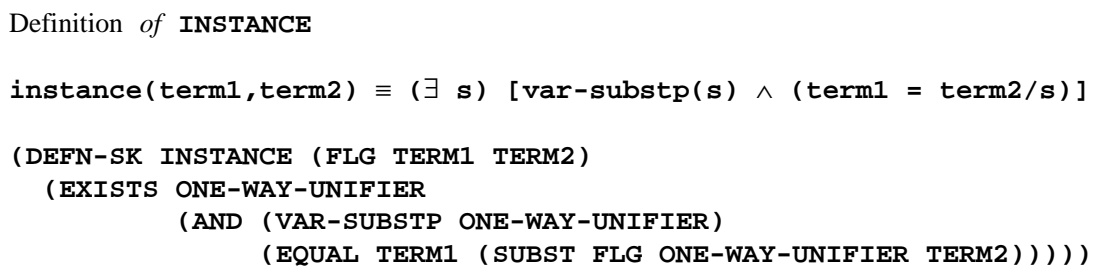

In fact the system adds the following axiom to "implement" this definition. The first conjunct gives a sufficient condition for TERM1 to be an instance of TERM2: if TERM1 is the result of substituting a variable substitution ONE-WAY-UNIFIER into TERM2, then TERM1 is an instance of TERM2. The second conjunct 
gives a necessary condition for TERM1 to be an instance of TERM2 (i.e. gives a consequence of instance (term1, term2)): if TERM1 is an instance of TERM2 then (ONE-WAY-UNIFIER FLG TERM1 TERM2) is a variable substitution such that TERM1 is the result of instantiating TERM2 with this substitution. Let us state the axiom both in informal and in formal notation. In the informal version we will write the second conjunct in the natural order rather than the contraposed order of the formal version (which is stated that way for technical reasons related to rewriting). The function ONE-WAY-UNIFIER is what is generally called a Skolem function, in that its only given property is that it provides a witness (in this case, to the existence of an appropriate substitution).

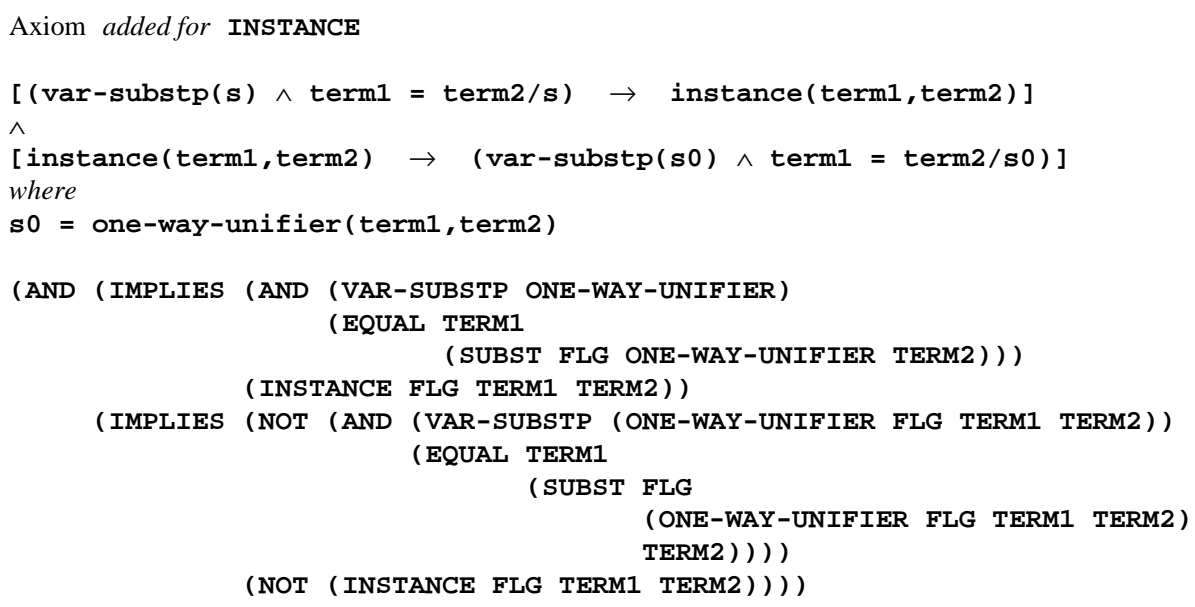

Our final definition from "terms.events" is rather idiosyncratic to the application at hand; it will be used to construct a substitution that is used in the proof of the main theorem. nullify-subst (s) is a substitution that maps the domain of $\mathbf{S}$ to the constant term (FN) .

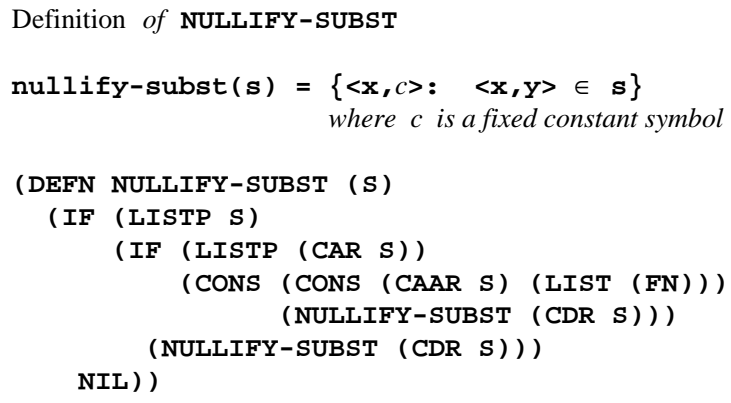

\section{Some Basic Supporting Lemmas}

In order to complete our mechanically-checked proof of the main theorem, we required a number of lemmas about the notions introduced above. We present some of those in this section, for two reasons. First, these lemmas give a flavor of the kinds of lemmas that appear in the libraries for this effort -- "sets.events", "alists.events", and "terms.events" -- and more generally, in other libraries as well. Second, we refer to these 
lemmas in some of the proofs that come later, but do not wish to clutter the exposition there with such trivial considerations. By the way, this is meant to be a representative list, not an exhaustive one.

The first lemma says that application of a substitution does not affect the domain.

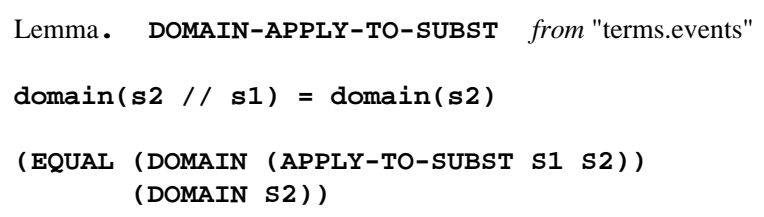

The next lemma says that a substitution has no effect when its domain contains no variables occuring in the term to which it is applied.

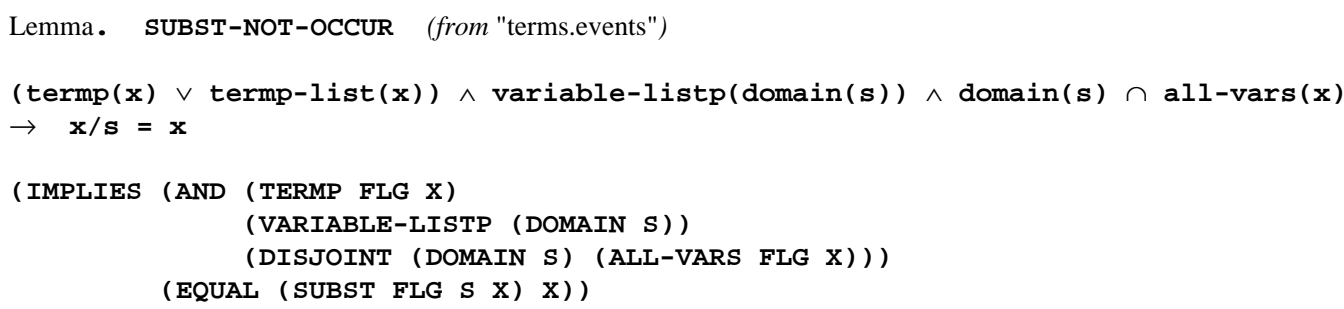

The following lemmas say that there is no effect when restricting (respectively, co-restricting) a substitition $\mathbf{s}$ to a subset $\mathbf{x}$, as long as all of the variables of the term term to which $\mathbf{s}$ is applied belong to (repectively, do not belong to) $\mathbf{x}$. (In fact, they say that it is sufficient that none of those variables belong to $\mathbf{x} \cap \operatorname{domain}(\mathbf{s})$.)

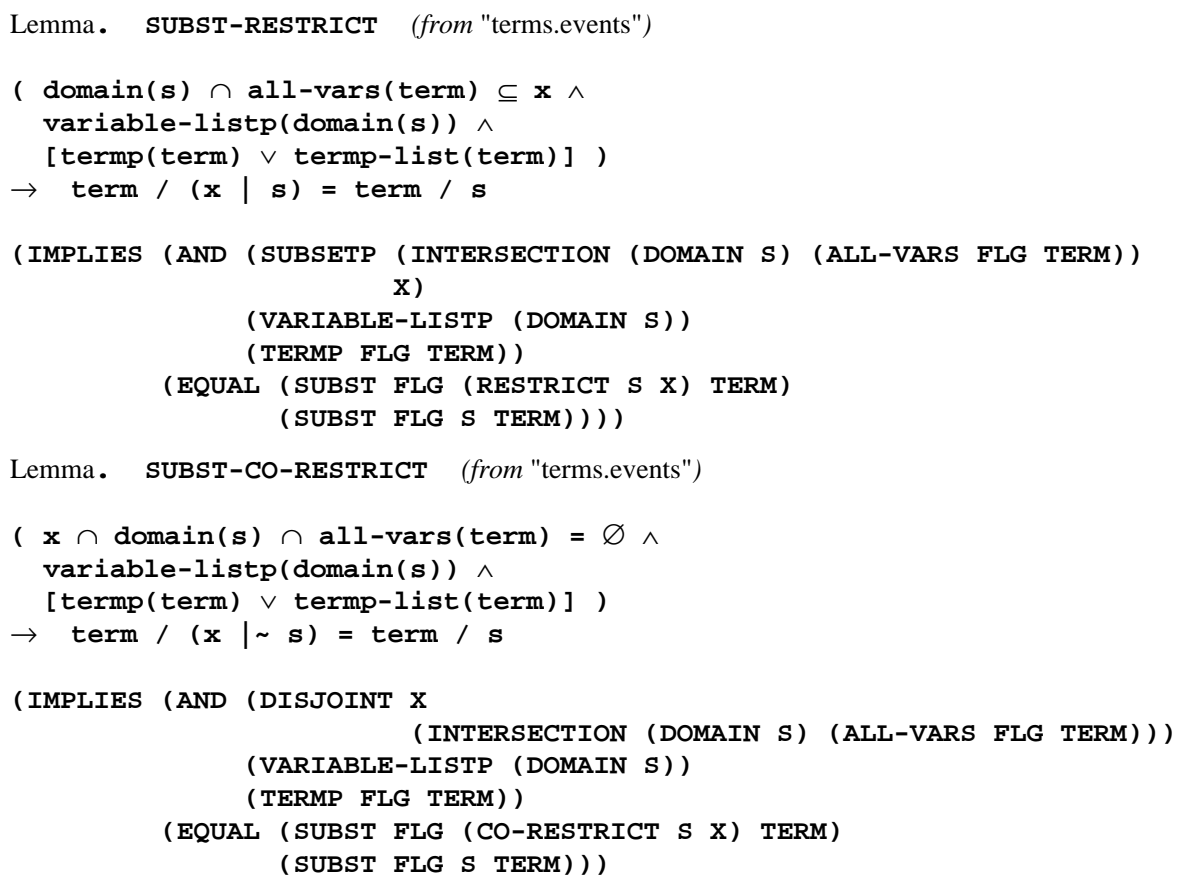


Two related lemmas say that one can drop a part of a subsitution whose domain does not intersect the term in question.

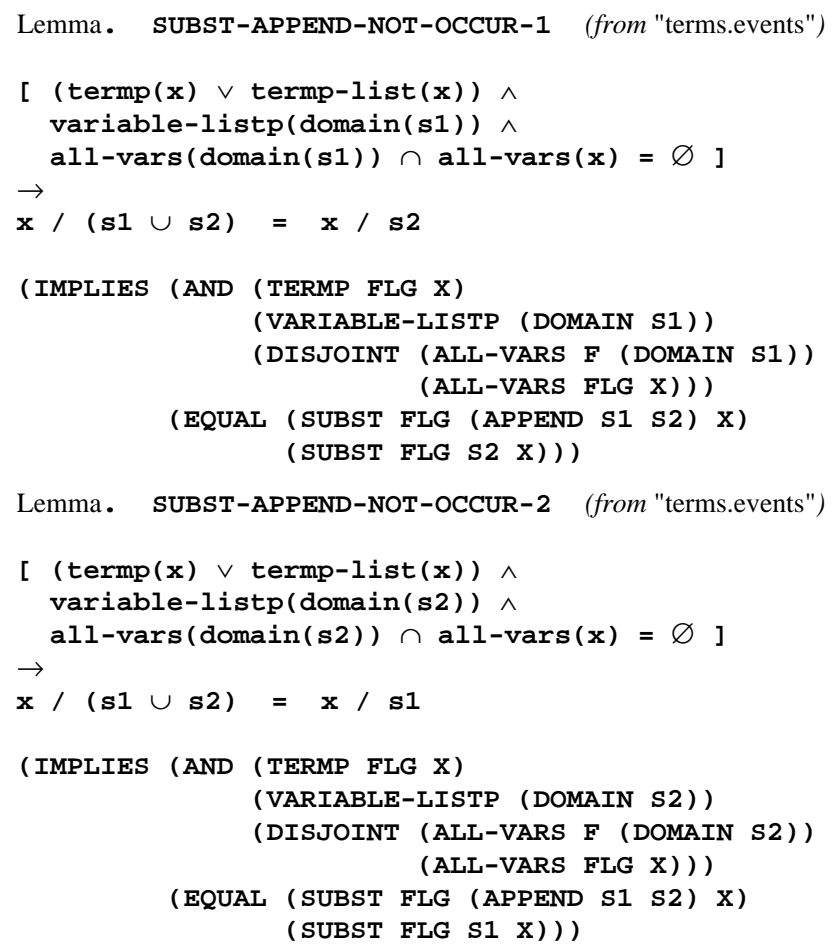

The following rewrite rule is kept in a disabled state, meaning that it is not used by the Boyer-Moore prover except when a hint is given to enable this rule. It is very useful when trying to prove that two lists do not intersect, because it reduces that problem to the problem of showing that nothing can belong to both lists. Functions such as DISJOINT-WIT are often called definable Skolem functions in that they provide witnesses to existential assertions (when they hold), in this case the assertion that $\mathbf{x}$ and $\mathbf{y}$ are not disjoint. ${ }^{6}$

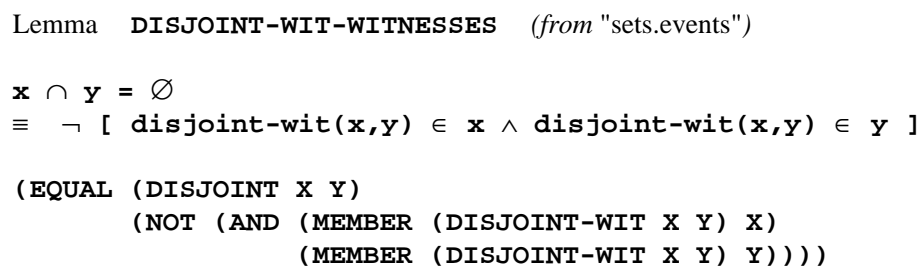

The following lemma points out the obvious relationship between the domain of a restriction with the domain of the given substitution.

\footnotetext{
${ }^{6}$ The function DISJOINT-WIT is actually defined by recursion in "sets.events". The idea of using definable Skolem functions in the Boyer-Moore prover was brought to our attention by Ken Kunen.
} 


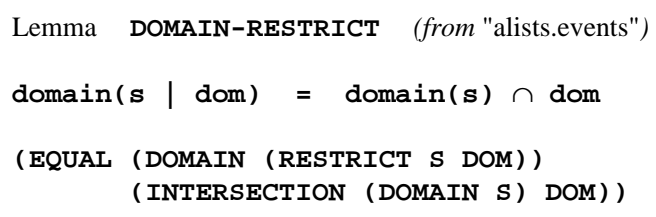

The remaining lemmas are also rather technical, so we prefer to liist them without comment here.

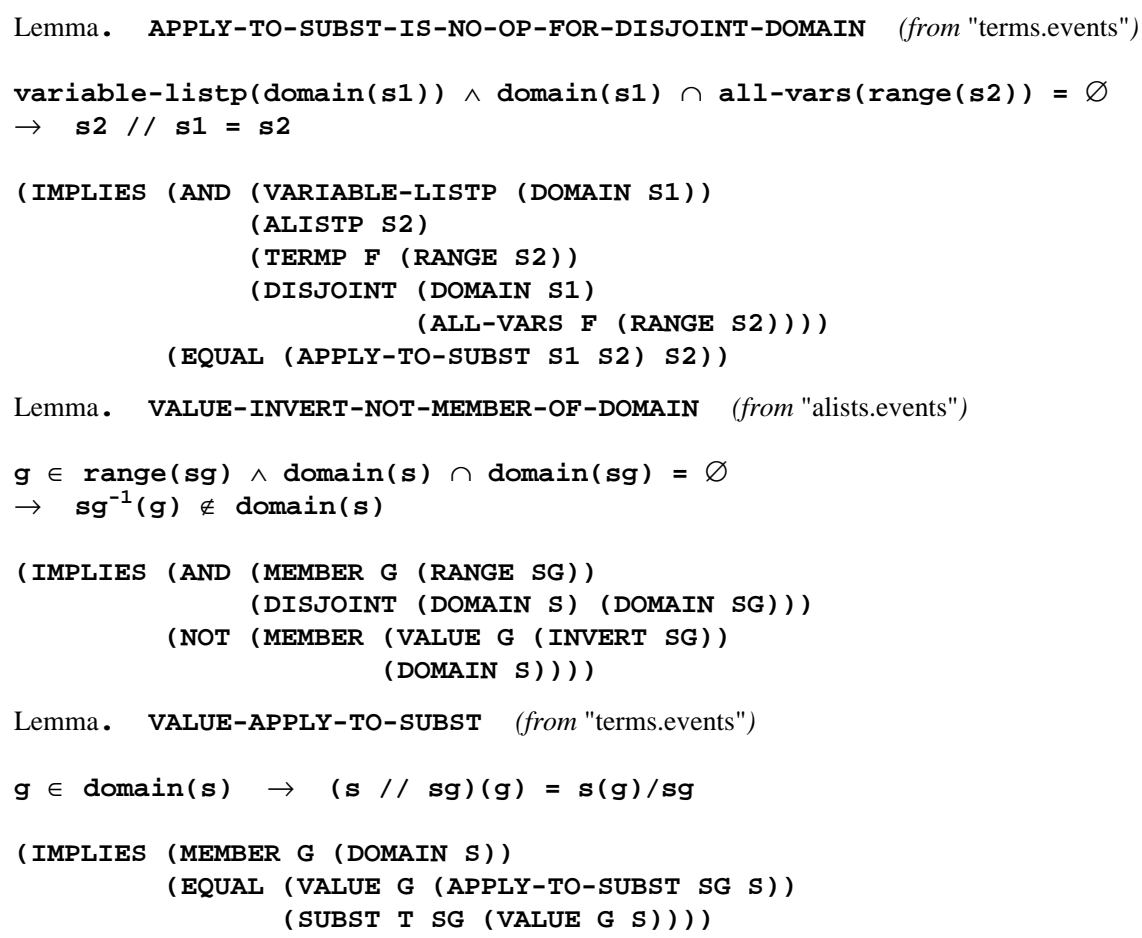

The following obvious fact says that NULLIFY-SUBST does not alter the domain of a given substitution.

Lemma. DOMAIN-NULLIFY-SUBST (from "terms.events")

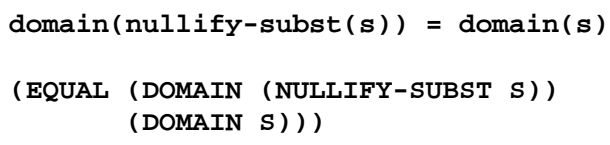

Here is another important property of NULLIFY-SUBST.

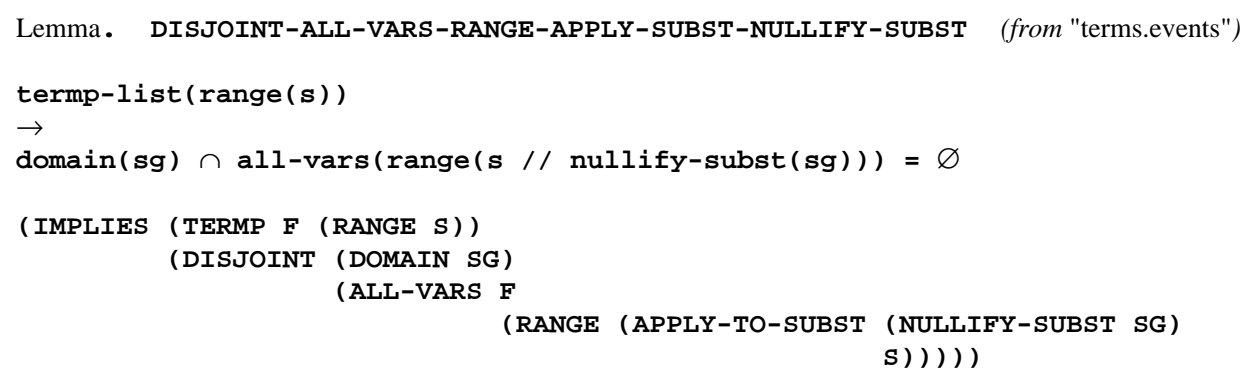




\section{Statement of the Main Theorem}

In this section we state our main theorem, which should perhaps be called a "metatheorem', since it's a theorem about formal theorems. The definitions in this section are all taken from the file "generalize.events", which is the last file in the Appendix. The events in that file have been numbered, and we give those numbers in the presentation below.

The first subsection below gives an outline of the high-level motivation for the definitions that follow. This is followed by a presentation of the definitions required for the statement of the main theorem. Some abbreviations are introduced in the third subsection. We conclude by stating the main theorem.

\subsection{Motivation}

In the introduction to this paper we discuss the original motivation for this work, which was to increase our confidence in the correctness of a particular algorithm for generalization in the presence of free variables. The following example is taken from the final section of [11]. It shows the necessity, for soundness, of having some restriction on how the GENERALIZE command interacts with the set of free variables of the proof state. Suppose that the history contains the rather silly (but correct) theorem that $[\mathbf{z + 1}<\mathbf{z} \rightarrow C]$ for some contradiction $C$. Then to prove $C$, it suffices to prove $[\mathbf{z}+1<\mathbf{z}$ ] for some $\mathbf{z}$. In fact $\mathbf{z}$ here is what we call a free variable in PC-NQTHM; this designation has the effect of allowing us to instantiate $\mathbf{z}$ to be anything we like. Of course there is no value of $\mathbf{z}$ for which the statement $[\mathbf{z}+\mathbf{1}<\mathbf{z}]$ is a theorem; there had better not be, or else $C$ would be a theorem! But suppose we allow ourselves to generalize this goal by replacing $\mathbf{z}+1$ by some new variable, say $\mathbf{a}$. The goal then is $[\mathbf{a}<\mathbf{z}$ ]. If $\mathbf{z}$ were still a free variable, then we could instantiate it to be $\mathbf{a}+1$, which would leave us with the goal [a $<\mathbf{a + 1}$ ]. But this goal is a theorem, which is supposed to imply that the original goal $C$ is a theorem -- yet, $C$ was chosen to be a contradiction!

One way around such a problem is to enforce the following rule: when generalizing with a substitution that replaces terms $\boldsymbol{t}_{\mathbf{i}}$ with corresponding new variables $\mathbf{v}_{\mathbf{i}}$ (e.g. replaces $\mathbf{z}+\mathbf{1}$ with $\mathbf{a}$ in the example above), the system removes from the list of free variables any variable that occurs in that substitution (e.g. $\mathbf{z}$ in that example). However, we can avoid removing quite that many free variables in general. The idea is that we must at least remove from the list of free variables those variables that occur both in the new current goal and in any of the terms being generalized away.

However, that set alone is not enough. Consider the theorem

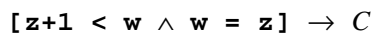


where as above, $C$ is contradictory. The to prove $C$ should be impossible, but we can do it if we can prove "appropriate" instances of the two goals $[\mathbf{z + 1}<\mathbf{w}]$ and $[\mathbf{w}=\mathbf{z}]$. Here "appropriate" means "via some substitution whose domain is contained in the set of free variables of the new proof state"; that set is $\{\mathbf{z}, \mathbf{w}\}$. After generalizing $[\mathbf{z + 1}]$ as in the previous example, we have the two goals $[\mathbf{a}<\mathbf{w}]$ and $[\mathbf{w}=\mathbf{z}]$. According to plan outlined just above, since $\mathbf{z}$ does not occur in the current goal $[\mathbf{a}<\mathbf{w}$ ] we may retain it on the list of free variables, and since $\mathbf{w}$ does not occur in the term $[\mathbf{z + 1}]$ that was generalized away we may retain it on the list too. But now if we instantiate both $\mathbf{w}$ and $\mathbf{z}$ with $\mathbf{a}+1$ then we can prove the resulting goals, a contradiction.

Here is an informal statement of the main result; a precise statement is of course the topic of the rest of this Section. This material is adapted from Subsection 4.3 of [11]. We defer to the proof presented in Section 5 below further motivation behind choices made here.

- Fix a proof state state, i.e. a list of terms (goals) together with a list of free variables.

- Let $\mathbf{s g}$ be a variable substitution.

- Let state' be the result of applying the GENERALIZE command, with substitution sg mapping new variables to terms. Thus, the new current (top) goal is the result of substituting the inverse $\mathbf{s g}^{\mathbf{- 1}}$ of $\mathbf{s g}$ into the current goal of state, and the remaining goals are unchanged.

- Let FREE and FREE' be the respective sets of free variables of state and state'.

- Consider the symmetric binary relation $\mathbf{R}_{\mathbf{0}}$ defined on $\mathbf{F R E E}$ as follows: $\mathbf{R}_{\mathbf{0}}(\mathbf{v}, \mathbf{w})$ if and only if $\mathbf{v}$ and $\mathbf{w}$ occur in a common goal of state' .

- Let $\mathbf{R}$ be the transitive closure of $\mathbf{R}_{\mathbf{0}}$.

- Let $\mathbf{C}$ be the range of $\mathbf{R}$ on the intersection of FREE with the variables of the current goal in state'.

- Let $\mathbf{v}$ be the set of variables that occur in the range of $\mathbf{s g}$.

In the second example presented above, $\mathbf{C}=\{\mathbf{z}, \mathbf{w}\}$ and $\mathbf{V}=\{\mathbf{z}\}$, so $\mathbf{C} \cap \mathbf{V}=\{\mathbf{z}\}$. With this example in mind, loosely speaking we want to remove from FREE the set (C $\cap$ V) consisting of all variables from FREE that both occur in somewhere in the terms being generalized away and also have "anything to do with" the new current goal (where "anything to do with" is defined in terms of the equivalence relation R). The precise relationship specified between FREE and FREE' is as follows.

FREE' $=($ FREE $\backslash(\mathrm{C} \cap \mathrm{V})) \backslash($ domain $\mathrm{sg})$

Here then, finally, is what we need to prove. It says that if the state is "valid" after generalization then it was already "valid", where "valid" is as explained in Subsection 1.3: some instance, where only free variables are instantiated, is a theorem. 
GENERALIZE SOUNDNESS THEOREM. Let $\mathbf{G}$ be the current goal in proof state state; let $\mathbf{P}$ be the conjunction of the rest of the goals of state; let sg be a substitution mapping some variables not occurring in state to terms; let $\mathbf{G}^{\prime}=\mathbf{G} / \mathbf{s g}^{-1}$ be the current goal in the new proof state state'; and let FREE and FREE' be the free variables of state and state', respectively. Suppose that for some substitution $\mathbf{s}^{\prime}$ with domain contained in $\mathbf{F R E E ^ { \prime }}, \mid-\left(\begin{array}{lll}\mathbf{G}^{\prime} & \boldsymbol{\&} & \mathbf{P}\end{array}\right) / \mathbf{s}^{\prime}$. Then for some substitution $\mathbf{s}$ with domain contained in FREE, we have $\mid-\left(\begin{array}{lll}\mathbf{G} \& \mathbf{P}\end{array}\right) / \mathbf{s}$.

An informal sketch of a proof of this theorem is outlined in [11]. Let us proceed with a careful and rather formal, but (we hope) motivated treatment.

\subsection{Definitions for main theorem}

Some terms are theorems relative to a given history. Here is the axiom that we introduce to capture the essence of "theoremhood"; in fact this is the only axiom we introduce about the notion of theorem. As in the introduction of the notions of variable and function symbol in Subsection 2.3, we use the CONSTRAIN mechanism to guarantee the consistency of these axioms. The first conjunct says that every theorem is a term. The second says that every instance of a theorem by a variable substitution is also a theorem.

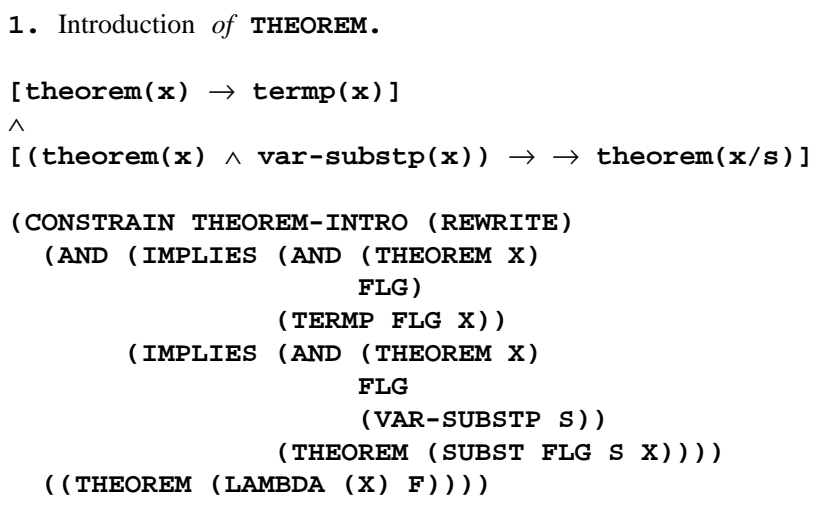

The corresponding notion of a list of theorems is obvious, and has properties (not listed here; see event \#3 in the Appendix) analogous to those for THEOREM in the event above.

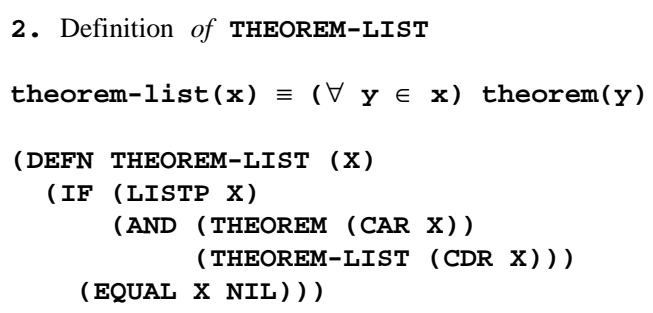

Next we wish to turn to the notion of a proof state, which is essentially a list of goals. We want to model 
a proof development methodology similar to the one in PC-NQTHM, as explained in the introduction, especially Subsection 1.3. That is, we model a proof state as an ordered pair (a LISTP) consisting of a term list (intuitively, a list of goals) together with a list of variables (intuitively, the free variables of that proof state).

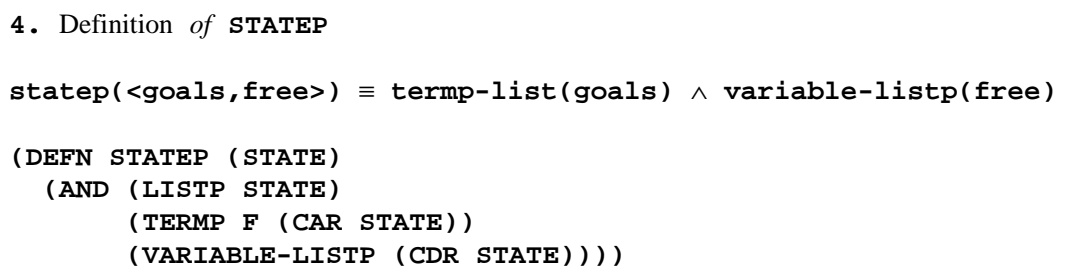

In order to state our main theorem we need a notion of valid state. This definition captures the corresponding notion defined in [11], namely, a valid state is a state with the following property: for some variable substitution on a subset of the free variables of the state, if one substitutes that substitution into the goals of the state then the results are theorems. (The event type "DEFN-SK' is discussed above with the definition of INSTANCE.)

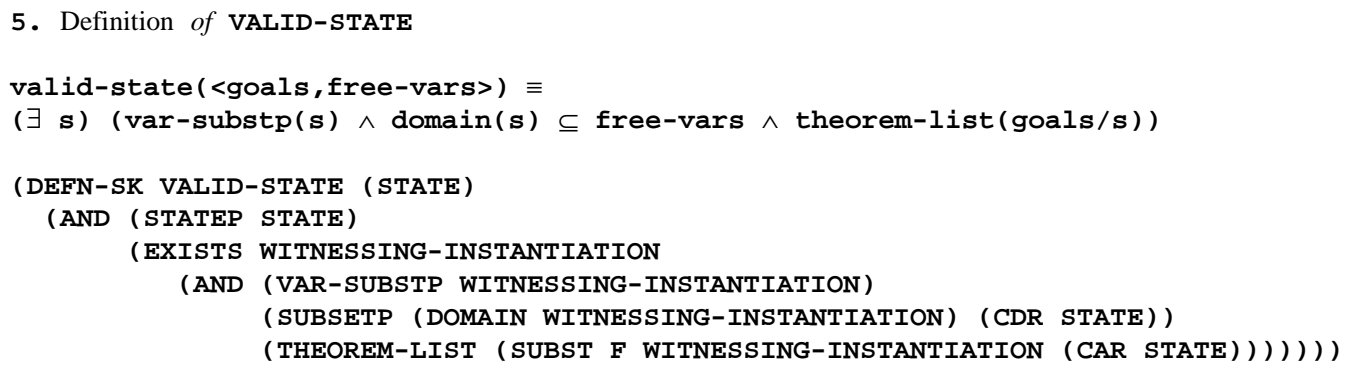

This definition adds a Skolem function WITNESSING-INSTANTIATION. This function may be thought of as picking out a substitution which, when applied to the goals of a given valid state, yields a list of theorems. Here is the axiom added by the system for the DEFN-SK event above. As with the previous DEFN-SK event introducing the function INSTANCE, this property of the witness is expressed by the second conjunct in the following axiom (which is stated in the contrapositive in the formal version, for technical reasons). 


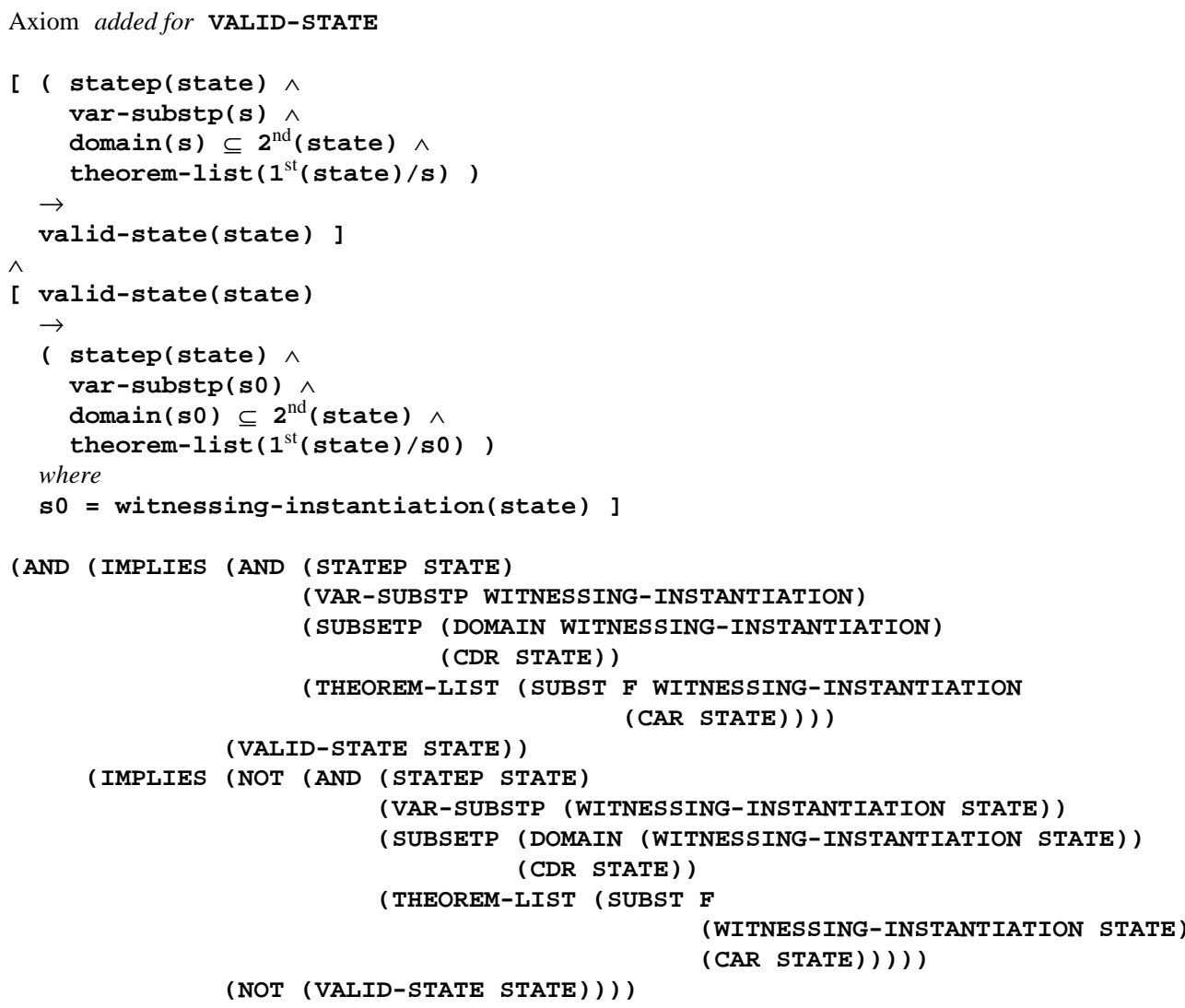

The following definition is auxiliary to GEN-CLOSURE. Informally, we can say that given a list $\mathbf{f}$ ree of "free variables" along with a list goals of terms and a list vars of variables (intuitively, a list of variables that we've constructed so far in our process of forming the closure), then new-gen-vars (goals, free, vars) is a list of those members of free that occur in a goal in goals that contains an occurrence of a variable in vars.

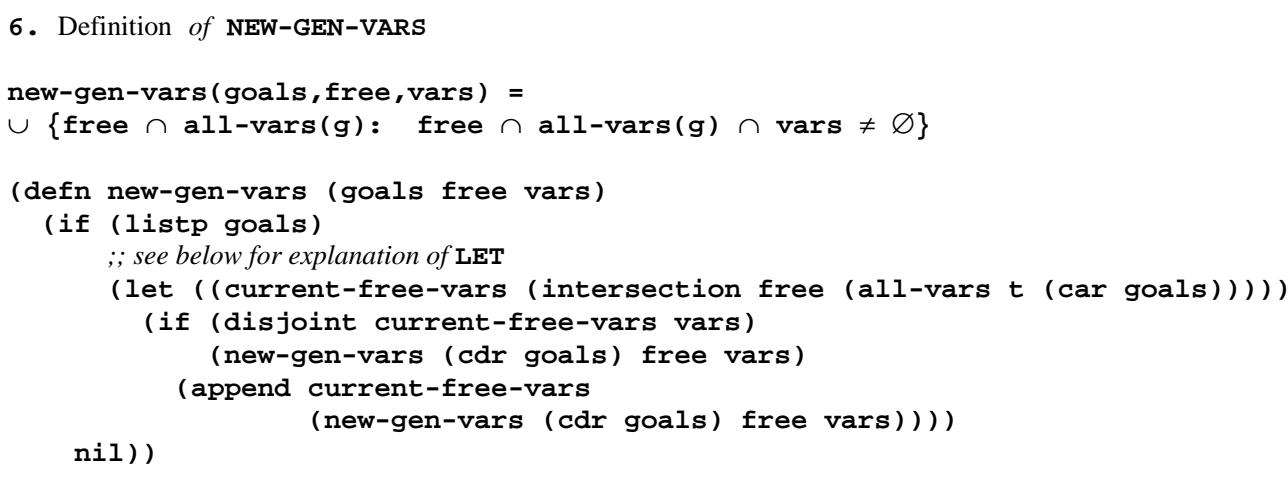

Notice the use of LET above. We use an extension to the syntax of the Boyer-Moore logic in which LET has the same meaning as it does in Common Lisp [17]; see the third appendix of the PC-NQTHM manual [10] for details. So for example, CURRENT-FREE-VARS in the definition above should be viewed as an abbreviation for (INTERSECTION FREE (ALL-VARS T (CAR GOALS))), 
i.e. for free $\cap$ all-vars $\left(1^{\text {st }}(\right.$ goals $\left.)\right)$.

Now we can define the closure referred to above. We may speak of gen-closure (goals, free, free-vars-so-far) as "the GEN-CLOSURE Of free-vars-so-far with respect to goals and free." The recursive nature of the definition of GEN-CLOSURE makes it a bit difficult to express informally; our apologies are probably in order for the rather obscure informal definition below.

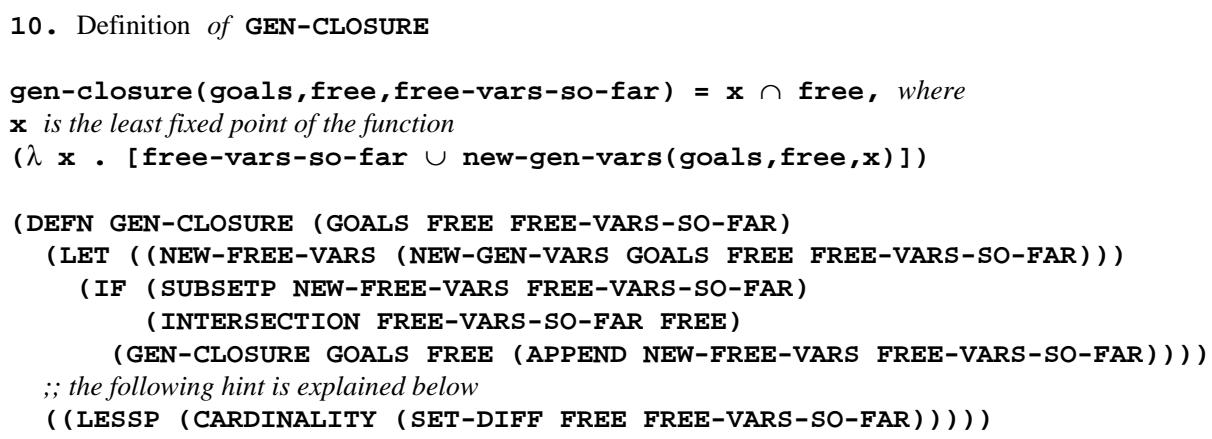

Notice that the definition above is recursive. The Boyer-Moore logic requires a proof in such cases; one might call this a 'termination proof', The proof obligation is actually completely precise and need not be understood in the context of termination of some execution, though that's a reasonable motivation. Informally speaking, the hint (LESSP (CARDINALITY (SET-DIFF FREE FREE-VARS-SO-FAR))) at the end of the 'DEFN', event above instructs the system to prove that the cardinality of (free \} \text { free-vars-so-far) } decreases on each recursive call of the function GEN-CLOSURE. Formally, the proof obligation in this case is as follows.

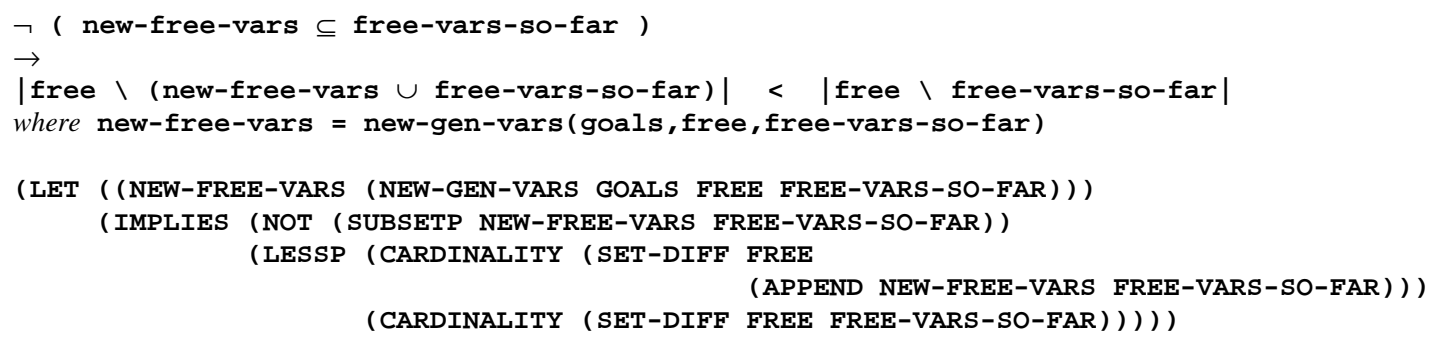

Inspection of the file "generalize.events" shows that a couple of lemmas were proved to help with the termination proof. In particular, the following lemma is easily proved by the system using induction. (A moment's reflection will suggest its utility in the proof of the termination goal displayed just above.)

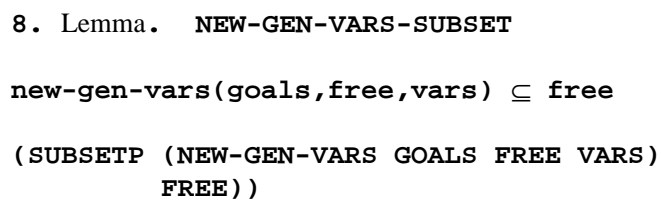


Now let us formalize the hypothesis under which the GENERALIZE command (to be defined shortly) is allowed to be executed. The GENERALIZE command is intended to apply the inverse of some variable substittuion sg to the top goal in the current proof state. Thus in the examples presented earlier in this section, the generalization obtained by replacing $\mathbf{z}+\mathbf{1}$ by $\mathbf{a}$ is represented by the variable substitution $\{<\mathbf{a}, \mathbf{z + 1 > \}}$. As for the other parameters below: state is a proof state, the domain of $\mathbf{s g}$ is disjoint from the variables occurring in the goals of the state, there is at least one goal in the state, and the domain of sg is disjoint from the free variables of the state. We take liberties in the informal version below by writing state as

<goals, free>.

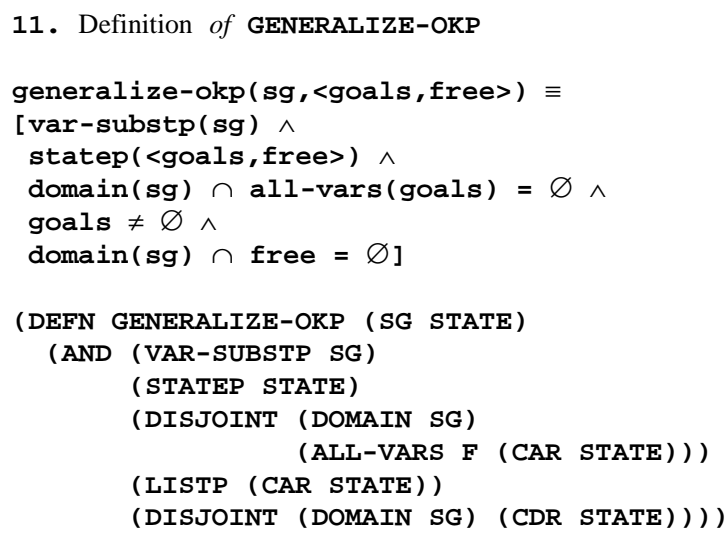

We define the function GENERALIZE to take a substitution sg and a proof state state and return a new proof state. ${ }^{7}$ The goals of the new proof state are the same as the goals of state except that the first (i.e. top, current) goal has been modified by substituting the inverse of the variable substitution sg into the first goal of state, and the list of free variables has been (possibly) reduced.

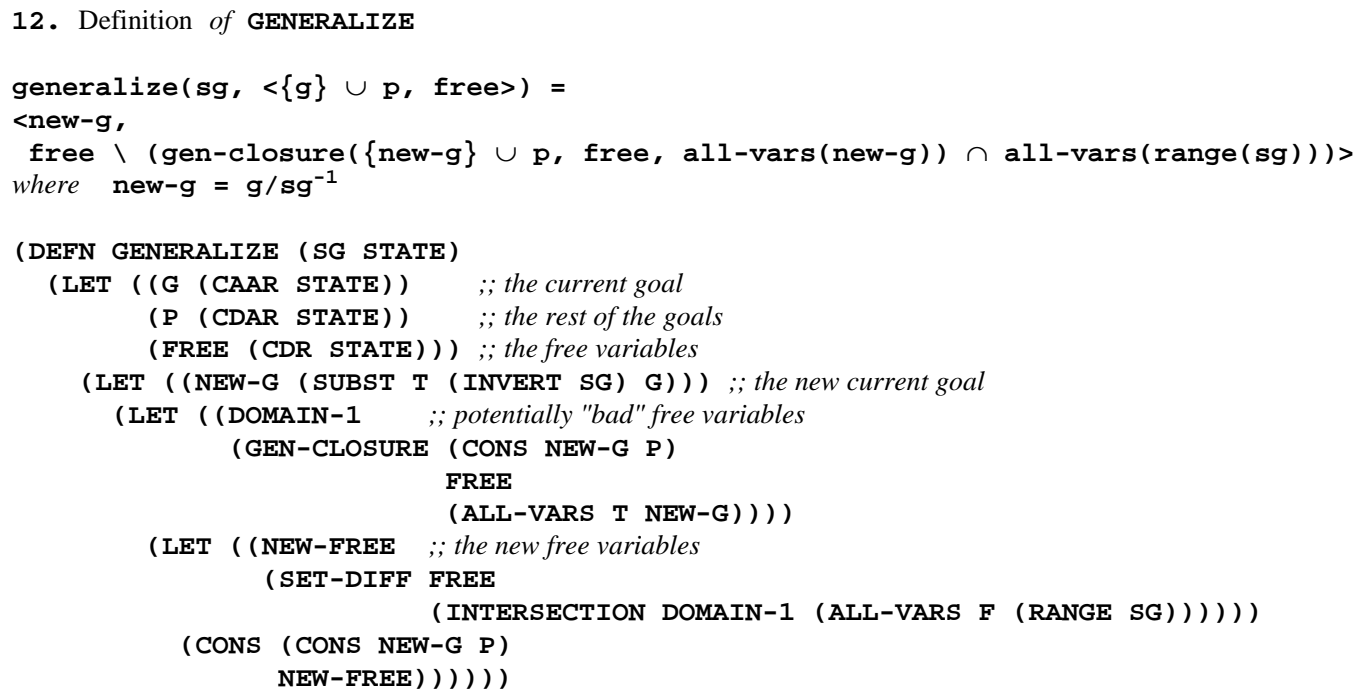

\footnotetext{
${ }^{7}$ The set DOMAIN-1 in the definition below is what is called $\mathbf{C}$ in 4.1 above; the name suggests (and is closely related to) the domain of a substitution $\mathbf{S} 1$ that appears later, during the proof.
} 


\subsection{Some abbreviations}

Before we state the main theorem, let us introduce some abbreviations for terms that occur repeatedly throughout the rest of this exposition. As usual, we'll use both informal notation and formal notation to introduce these abbreviations. Abbreviations will appear in italics font.

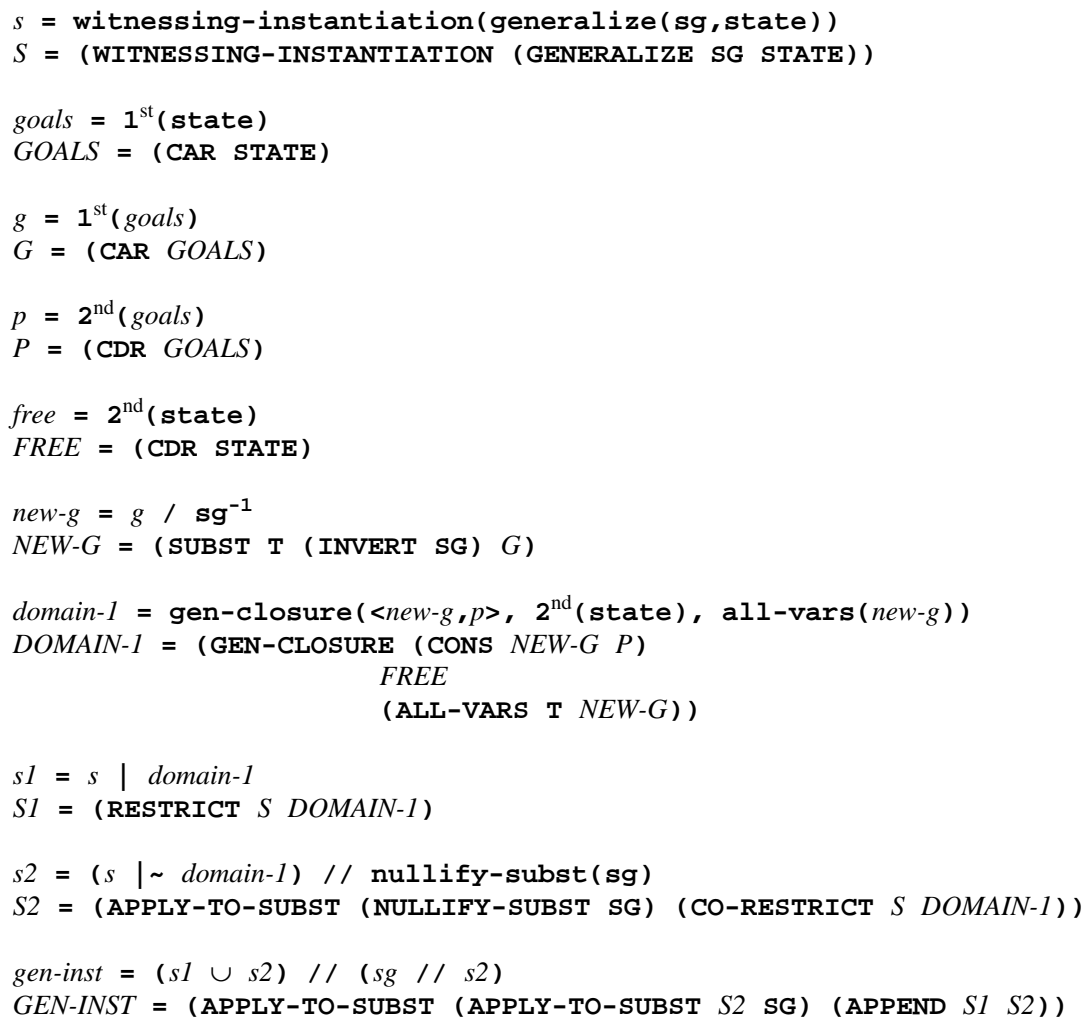

Let us use the abbreviations introduced above to restate the definition of GENERALIZE.

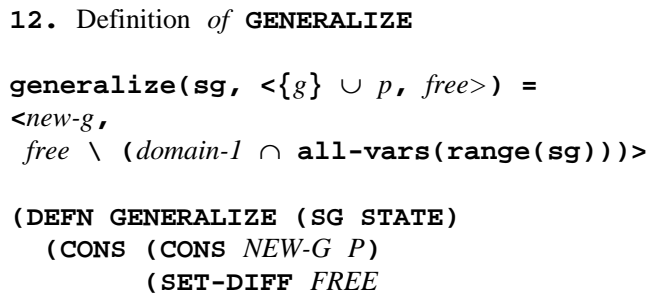

\subsection{Statement of main theorem}

Finally we can state the main theorem. It says that if the preconditions of the GENERALIZE command are met and if the result of applying this command is a valid proof state, then the original proof state is valid. 


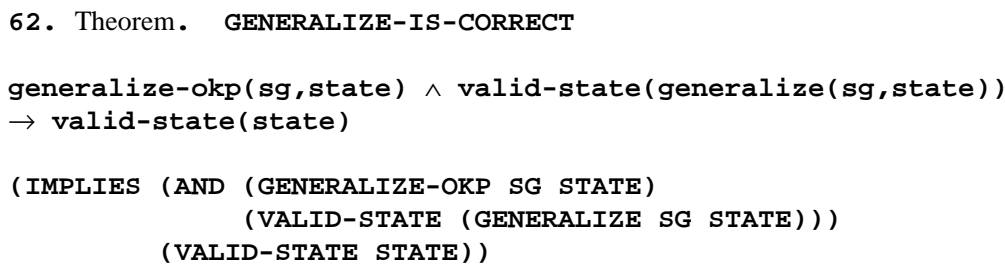

\section{Proof of the Main Theorem}

In this final section we outline the mechanically-checked proof of the main theorem GENERALIZE-IS-CORRECT displayed above. We actually break this proof into three parts. First we show how to reduce the main theorem to two lemmas. Then we devote the remaining two subsections to the respective proofs of those two lemmas.

\subsection{Reducing the theorem to two lemmas}

First of all, notice that by definition of VALID-STATE it suffices to find some substitution, call it gen-inst (sg, state), for which we can prove the following fact.

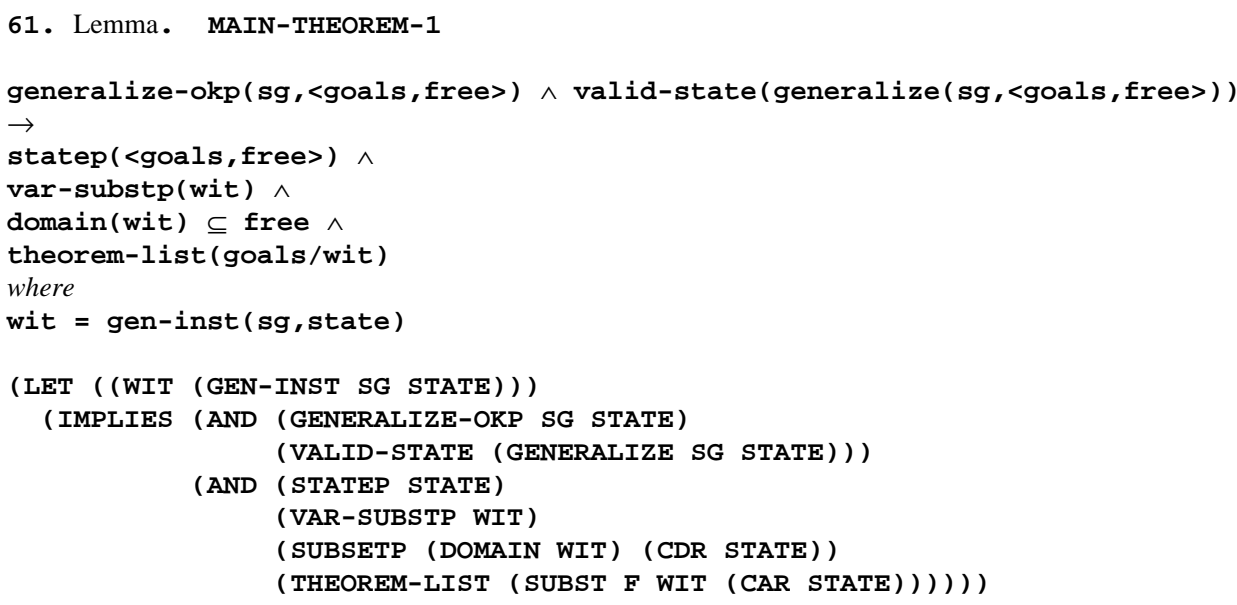

Such a variable substitution wit $=$ gen-inst (sg, state) can be constructed as follows (see also Subsection 4.1 for motivation). Let $\mathbf{s}$ be a variable substitution that witnesses the validity of the state generalize (sg, state). Let domain-1 be the GEN-CLOSURE of the variables occurring in the new current goal (which is the result of applying the inverse of the generalizing substitution to the current goal), with respect to the new goals and the existing list of free variables. Then the desired substitution gen-inst (sg, state) may be defined as follows. 


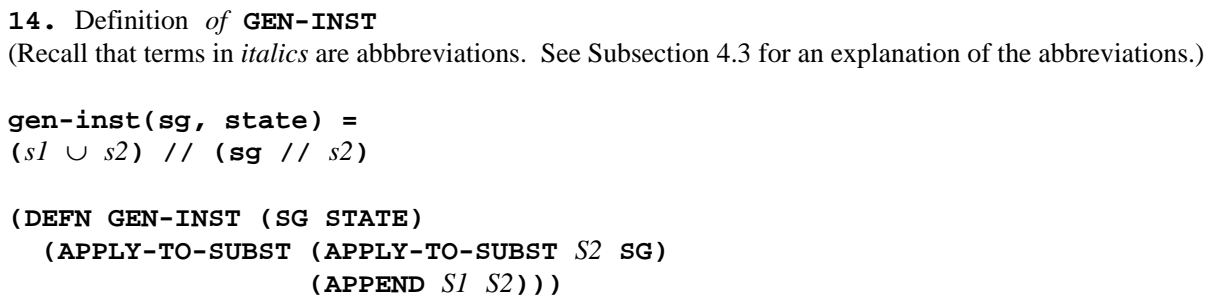

The first three conjuncts of the conclusion of MAIN-THEOREM-1 are now quite trivial; they correspond to events \#15, \#17, and \#19 in the numbered list of events from "generalize.events" in the Appendix (and are named MAIN-THEOREM-1-CASE-1, MAIN-THEOREM-1-CASE-2, and MAIN-THEOREM-1-CASE-3). The first of these, statep (state), is clear by definition of GENERALIZE-OKP. The second, var-substp (gen-inst (sg, state)), is clear from the way that gen-inst (sg, state) is built from variable substitutions. The third, domain (wit) $\subseteq 2^{\text {nd }}$ (state), is also straightforward, though (like many simple results proved with the Boyer-Moore prover) it uses basic "library" facts such as the lemma DOMAINAPPLY-TO-SUBST (see Section 3 above). A key observation for that case, which is specific to our notion of generalization, is the fact that the set of free variables of the state obtained by applying the GENERALIZE command is a subset of the set of free variables of the original state:

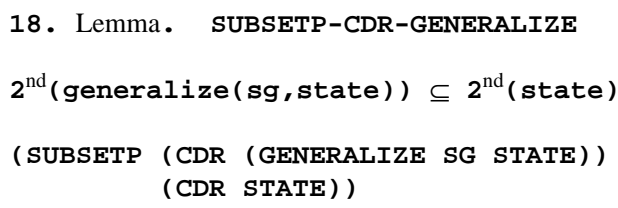

It remains then only to check the last of the four cases from the conclusion of MAIN-THEOREM-1, i.e. to prove the following (stated using abbreviations, in italics, from Subsection 4.3).

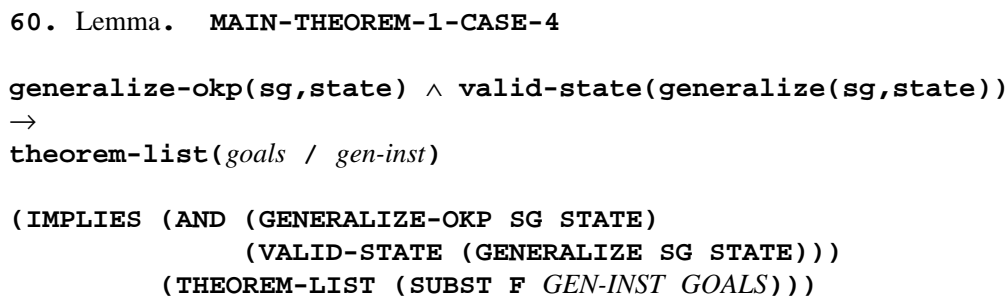

The idea now is to introduce a new predicate MAIN-HYPS that that abstracts the hypotheses that are needed, and then split the proof into two parts. First, we show that MAIN-HYPS implies the result for arbitrary substitutions and goals. Second, we show that MAIN-HYPS holds of the particular substitutions and goals in question. Thus the first part, MAIN-HYPS-SUFFICE below, states that the bizarre-looking substitution ( ( $\mathbf{1}$ $\cup$ s2) // (sg // s2)) (which however is closely related to the definition of GEN-INST) serves to 
create a list of theorems, assuming that MAIN-HYPS holds of the relevant substitutions and goals. The other part, MAIN-HYPS-RELIEVED, shows that MAIN-HYPS holds of the necessary substitutions and goals.

Notice that we do not use abbreviations in the first of the following lemmas; as suggested above, it holds of arbitrary substitutions and goals. However, it is applied (by the theorem prover's rewriter) under the particular instantiation $\{\mathbf{S} 1:=S 1, \mathbf{S 2}:=S 2$, GOALS $:=G O A L S\}$.

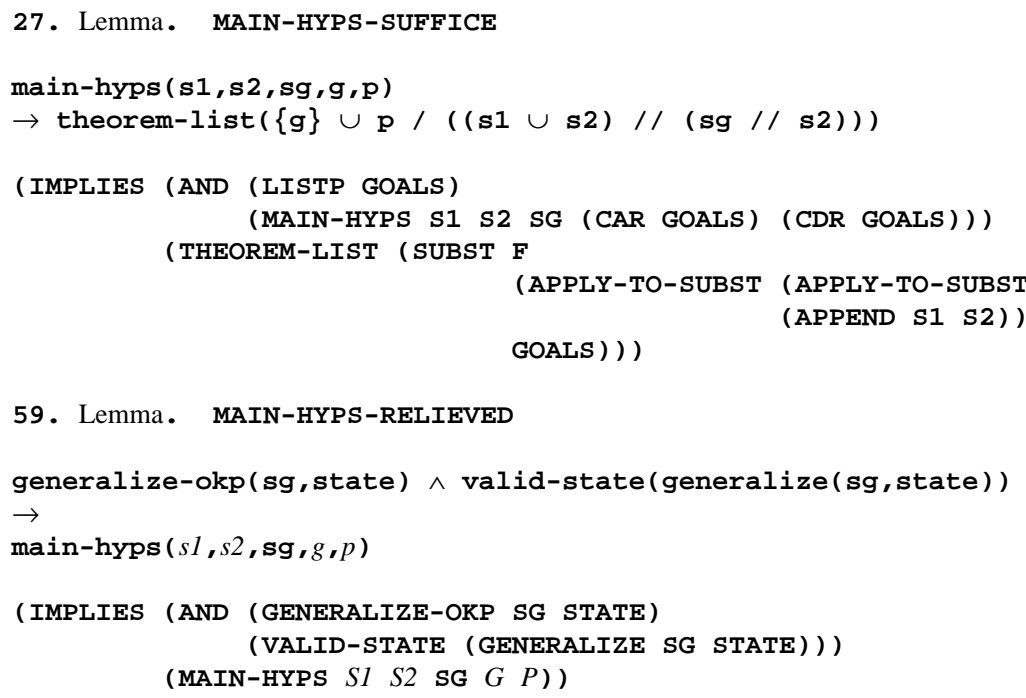

The proof now naturally splits into two parts, one for each of the two lemmas displayed immediately above. We close this subsection with the remaining definitions before turning to the proofs of these two remaining lemmas in the respective subsections below. First, here is the definition of MAIN-HYPS.

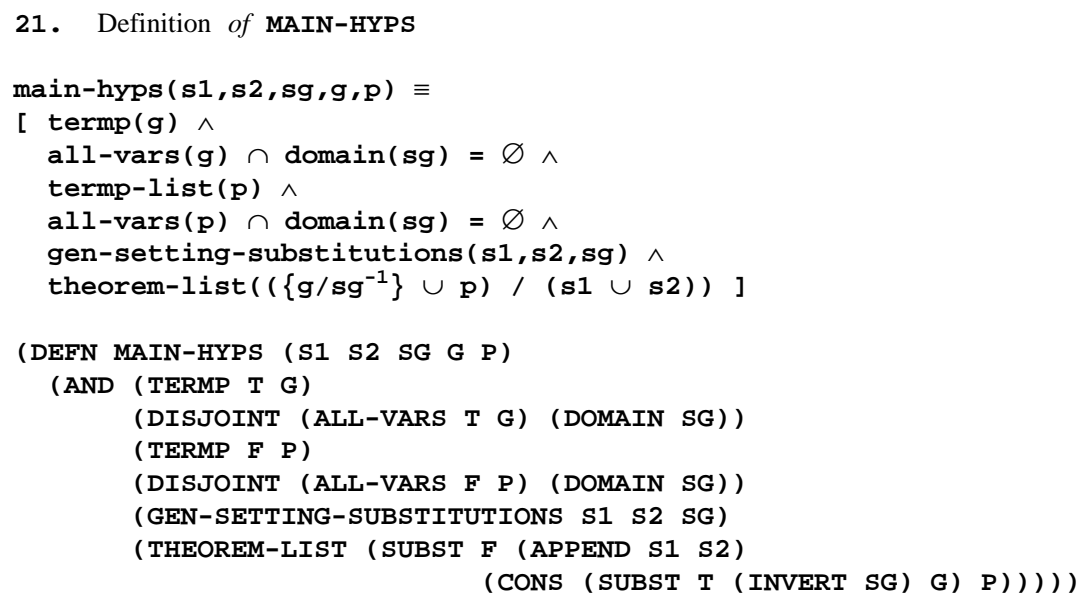

The auxiliary function GEN-SETTING-SUBSTITUTIONS is defined as follows. 
20. Definition of GEN-SETtING-SUBStitutions

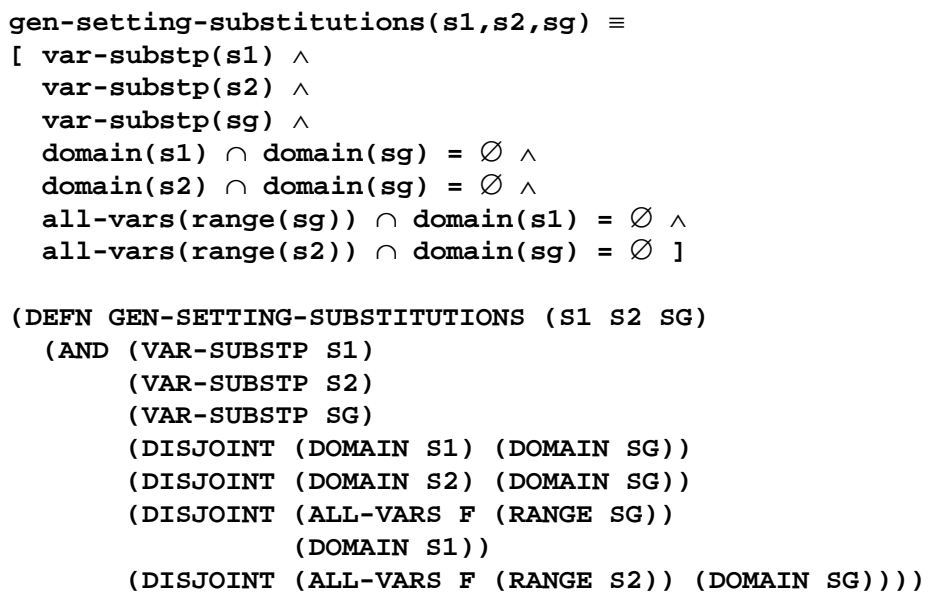

\subsection{Proof of the lemma MAIN-HYPS-SUFFICE}

Let us state the lemma once again.

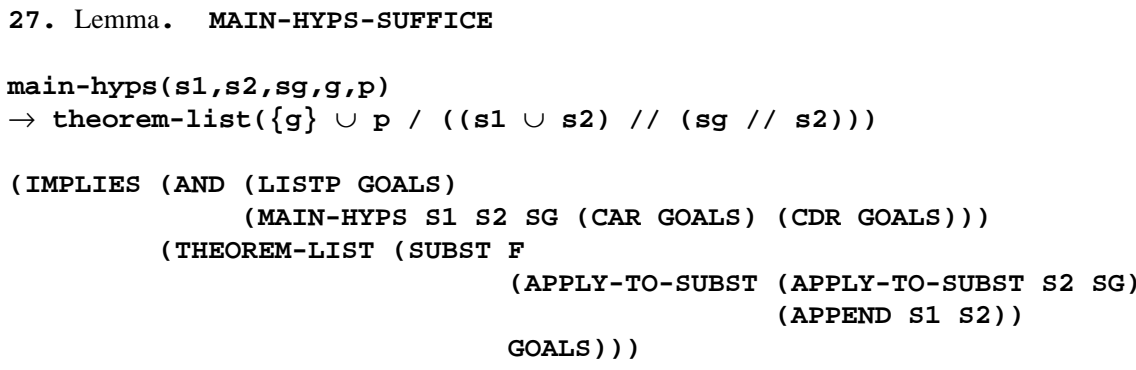

27. Lemma. MAIN-HYPS-SUFFICE

If we apply the definitions of SUBST and THEOREM-LIST in the expression above, then we see that it suffices to prove the following two properties. (Think of $\mathbf{g}$ as the current goal and of $\mathbf{p}$ as the rest of the goals.)

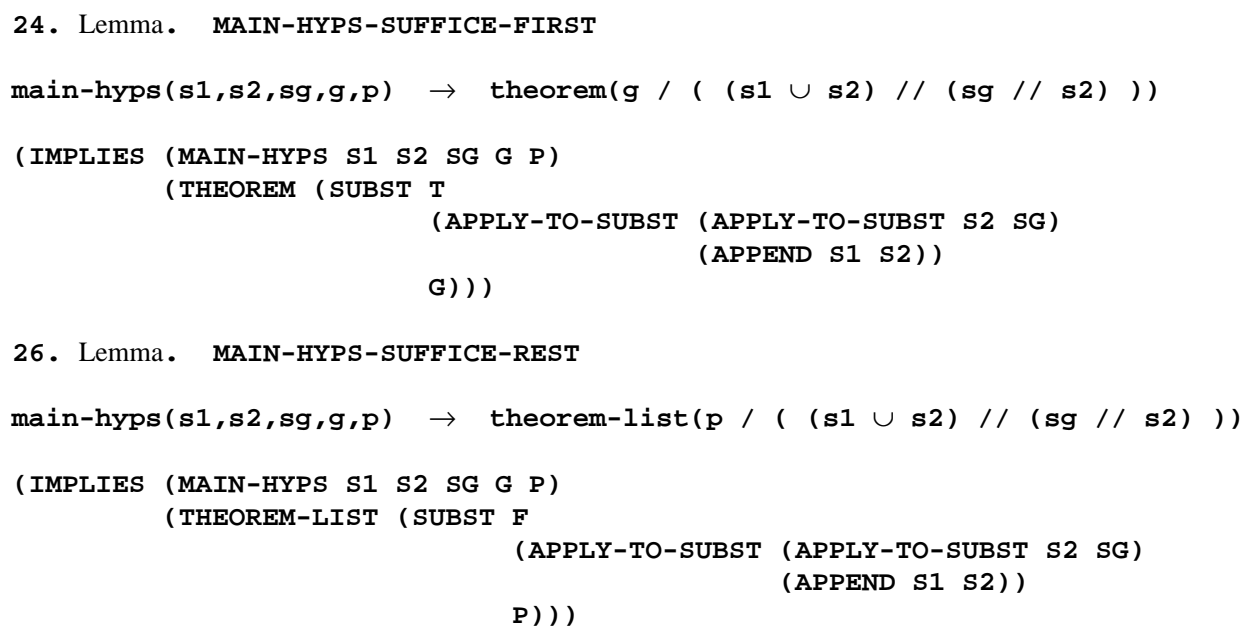

Let us consider these two cases separately. 


\subsubsection{Proof of the lemma MAIN-HYPS-SUFFICE-FIRST}

Consider the first of these two lemmas, MAIN-HYPS-SUFFICE-FIRST. Let us begin by arguing informally for its correctness. The last conjunct of MAIN-HYPS implies, assuming the hypothesis of the lemma, that $\left(\mathrm{g} / \mathbf{s g}^{-1}\right) /(\mathbf{s} 1 \cup \mathbf{s} 2)$ is a theorem. Now every instance of a theorem by a variable substitution is a theorem (by the CONSTRAIN event THEOREM-INTRO, event \#1 in "generalize.events"). Then MAIN-HYPS-SUFFICE-FIRST above follows if we can show that the proposed theorem is an instance of $\left(\mathrm{g} / \mathbf{s g}^{-1}\right) /(\mathbf{s} 1 \cup \mathbf{s} 2)$. The following lemma therefore implies MAIN-HYPS-SUFFICE-FIRST.

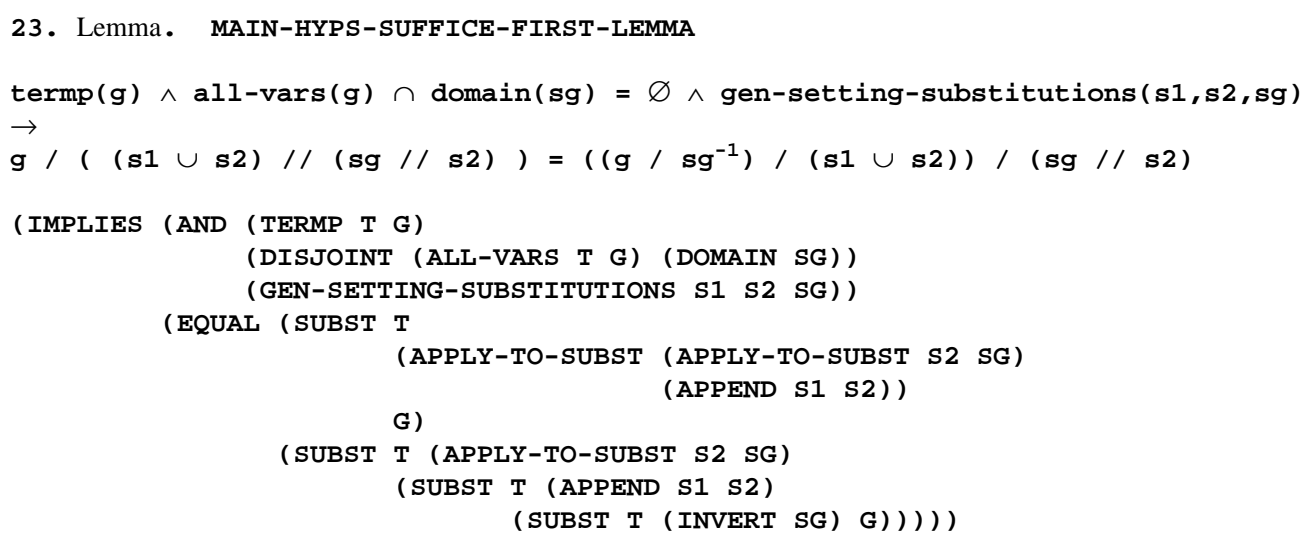

Let us see why this lemma holds, and in doing so, discover some of the motivation for the properties embodied in MAIN-HYPS. Assume the following hypotheses, the last of which is the inductive hypothesis. Note: we'll see during the proof what we need here about GEN-SETTING-SUBSTITUTIONS.

termp (g)

$\operatorname{all-vars}(g) \cap \operatorname{domain}(\mathrm{sg})=\varnothing$

gen-setting-substitutions (s1, s2, sg)

(IH) $g^{\prime} /((s 1 \cup s 2) / /(s g / / s 2))$

$=\left(\left(g^{\prime} / s g^{-1}\right) /(s 1 \cup s 2)\right) /(s g / / s 2)$

for all subterms $\mathbf{g}^{\prime}$ of $\mathbf{g}$

The proof now breaks into three cases. We omit a few details but give many others, just to show the kind of considerations required in the mechanically-checked proof.

Case 1: $\mathbf{g} \in$ range (sg), say $\mathbf{g}=\mathbf{s g}(\mathbf{v})$. Then we have, working with the right side of the goal equation: 


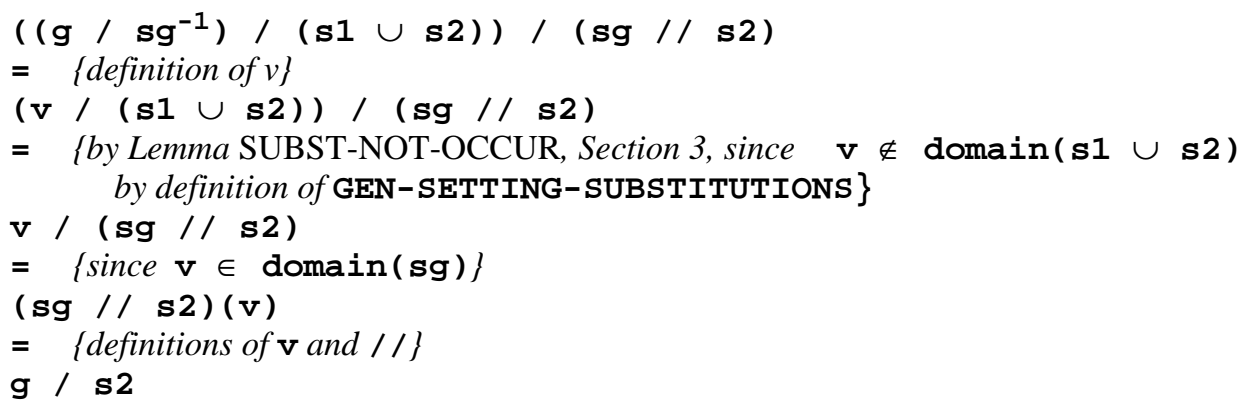

On the other hand, reducing the left side of the goal equation we have

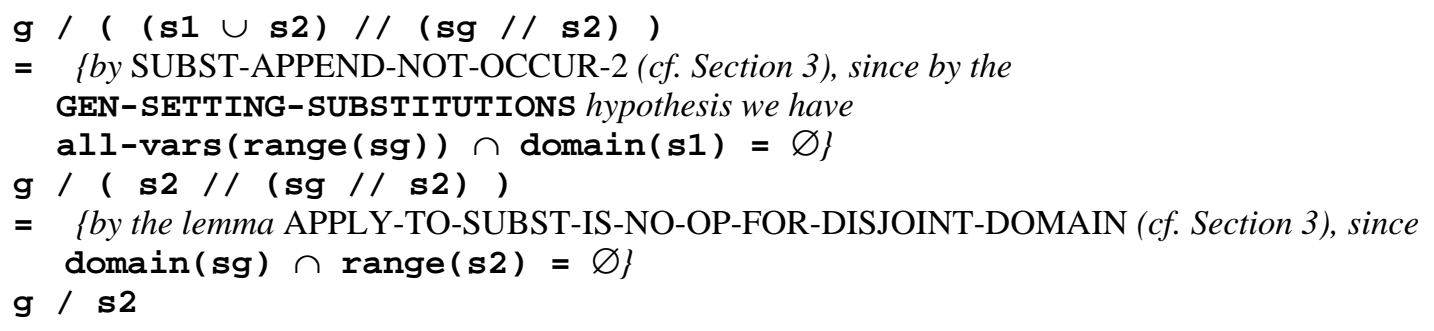

Case 2: $\mathbf{g} \notin$ range (sg) and variablep ( $\mathrm{g})$.

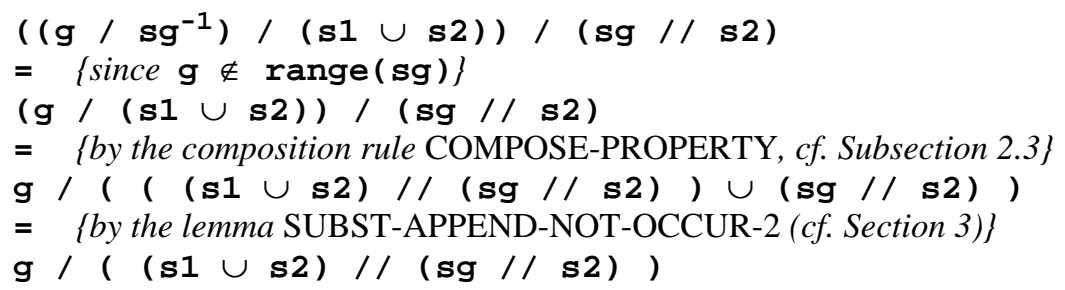

Case 3: otherwise. Then we may write $\mathbf{g}$ as $\left\langle\mathbf{f} \mathbf{v}_{1} \ldots \mathbf{v}_{\mathbf{n}}\right\rangle$, and we have:

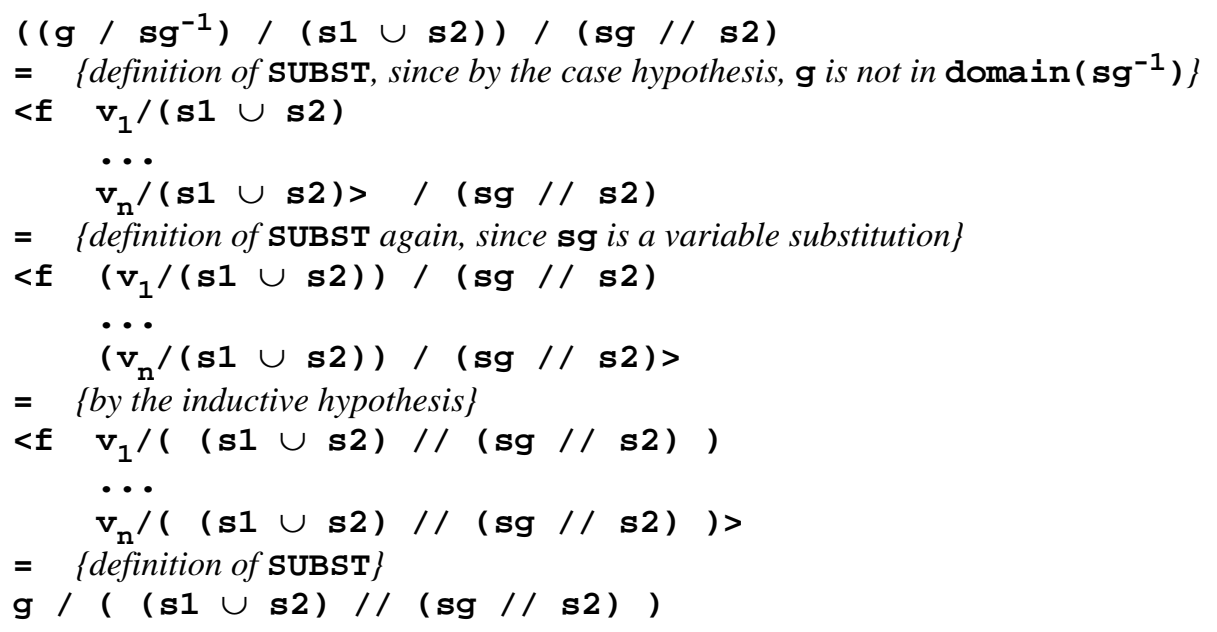

Actually, a formalization of this proof in the Boyer-Moore logic tends to require one to prove the similar 
theorem about lists of terms by a simultaneous induction. The theorem prover essentially carries out the above argument in proving event \#22 in "generalize.events", MAIN-HYPS-SUFFICE-FIRST-LEMMA-GENERAL, which is a generalization we provide of MAIN-HYPS-SUFFICE-FIRST-LEMMA to both terms and term lists. (That is, we leave $\mathbf{f l g}$ uninstantiated.) The cases in the inductive argument correspond to the definition of SUBST, so we supply the hint (INDUCT (SUBST FLG SG-1 G)) for this lemma. Notice that we also give $\mathbf{s g}^{-1}$ a name, $\mathbf{s g} \mathbf{- 1}$, for the technical reason that such induction hints in the Boyer-Moore prover must have variables in the argument positions.

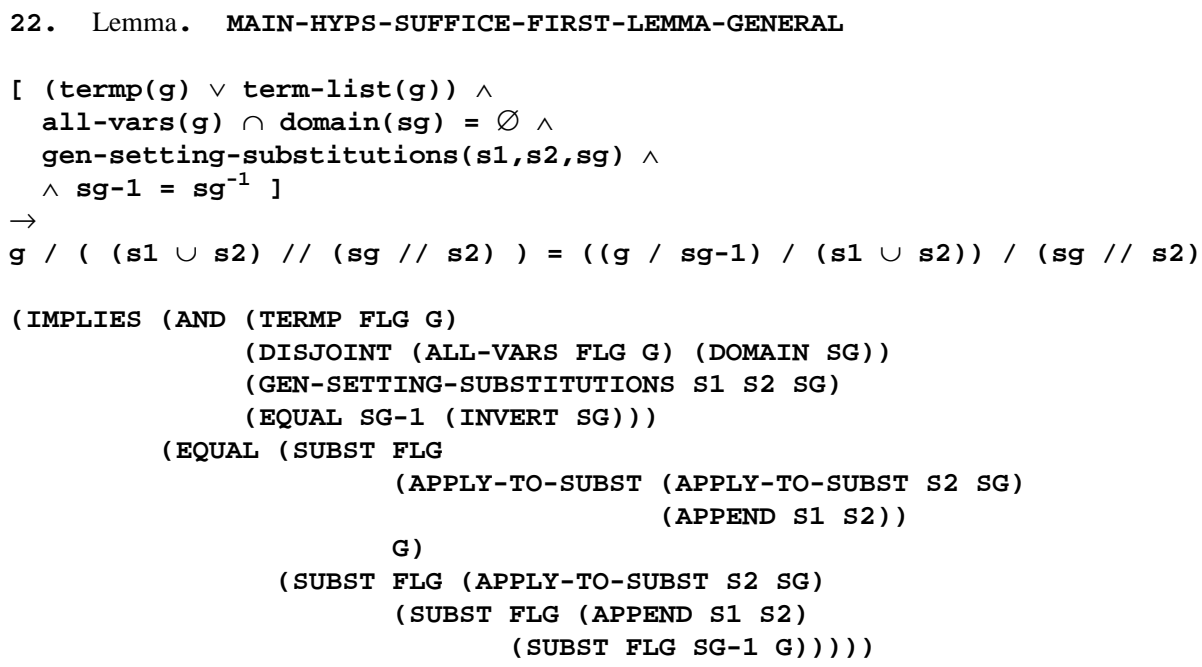

Finally, let us note that a number of trivial considerations that were ignored here must be dealt with in the mechanical proof. Consider again, for example, the second step in the proof of the first case above:

$(v /(s 1 \cup s 2)) /(s g / / s 2)$

$=\quad$ by Lemma SUBST-NOT-OCCUR, Section 3, since $\mathbf{v} \notin \operatorname{domain}(\mathbf{s} 1 \cup \mathbf{s} 2)$ by definition of GEN-SETTING-SUBSTITUTIONS \}

$v /(s g / / s 2)$

Why do we know that $\mathbf{v} \notin \operatorname{domain}(\mathbf{s} 1 \cup \mathbf{s 2})$ ? The reason above is "by definition of GEN-SETTING-SUBSTITUTIONS', If we think carefully here then we realize that this use of the definition of GEN-SETTING-SUBSTITUTIONS guarantees that the domain of $\mathbf{s g}$ is disjoint from the domains of $\mathbf{s} 1$ and $\mathbf{s} 2$; so at the very least we need to know that $\mathbf{v}$, i.e. $\mathbf{s g}^{\mathbf{- 1}}(\mathbf{g})$, is a member of the domain of $\mathbf{s g}$. A lemma to this effect, VALUE-INVERT-NOT-MEMBER-OF-DOMAIN, has been included in Section 3 . Another example where we glossed over small details is in the following step from Case 1:

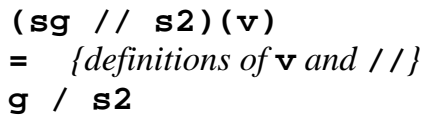

In fact we proved a lemma to accomplish this bit of reasoning; see VALUE-APPLY-TO-SUBST in Section 3 (with the confusing instantiation $\mathbf{g}:=\mathbf{v}, \mathbf{s g}:=\mathbf{s} 2, \mathbf{s}:=\mathbf{s g}$ ). 


\subsubsection{Proof of the lemma MAIN-HYPS-SUFFICE-REST}

Recall that the other half of proving MAIN-HYPS-SUFFICE is:

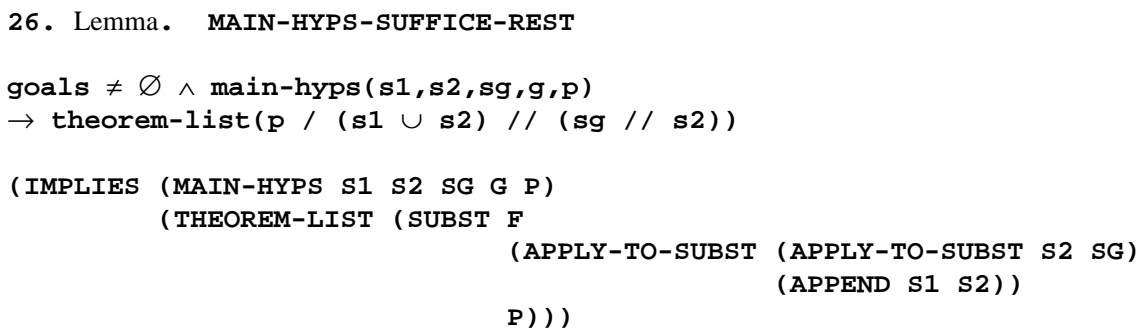

Again we may use the property that an instance of theorem (or theorem list) is a theorem (or theorem list, respectively). Therefore the property above follows from the following lemma, with FLG set to $\mathbf{F}$ and $\mathbf{S}$ set to (APPEND S1 S2) (informally, s1 $\cup \mathbf{s 2}$ ), together with the definition of MAIN-HYPS.

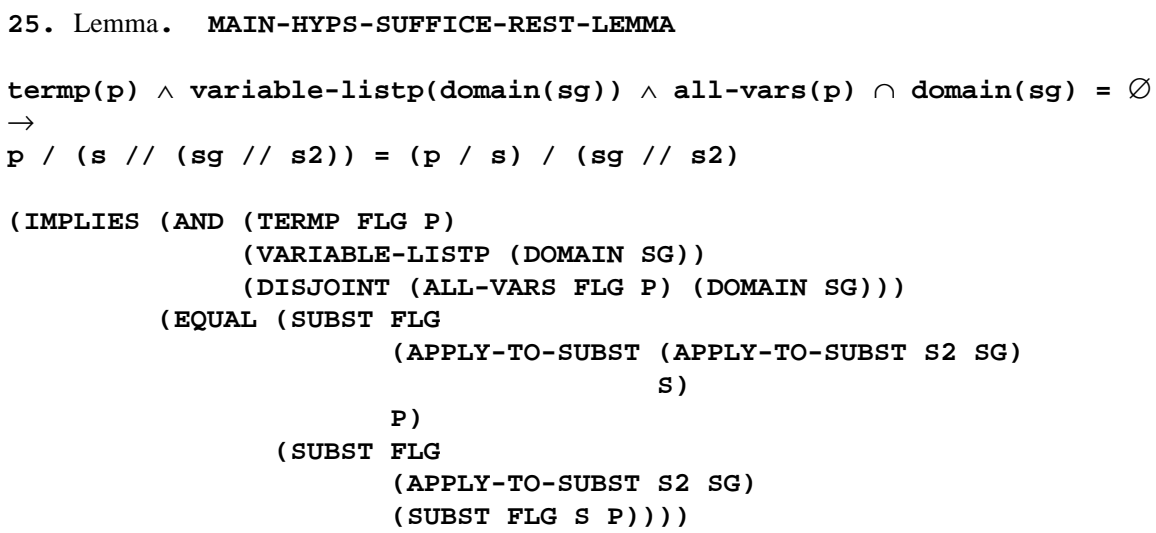

This is actually quite a straightforward result, using the rewrite rule COMPOSE-PROPERTY displayed in Subsection 2.3 above. Here is an informal sketch of the proof (but note that the theorem prover proves this automatically from the previously proved rules).

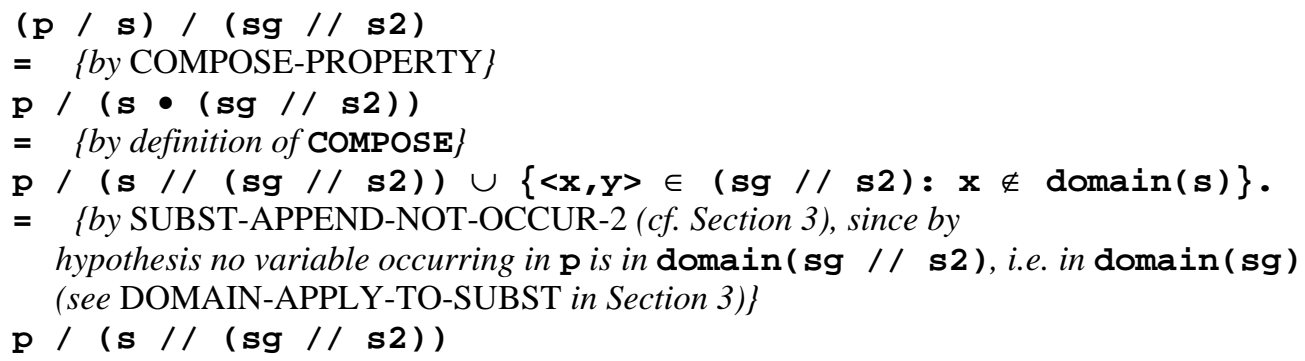

\subsection{Proof of the lemma MAIN-HYPS-RELIEVED}

The only thing left to prove is the lemma MAIN-HYPS-RELIEVED. Let us repeat the statement of that lemma, but opening up the definition of MAIN-HYPS. We will continue to use the abbreviations introduced in Subsection 4.3 above. 


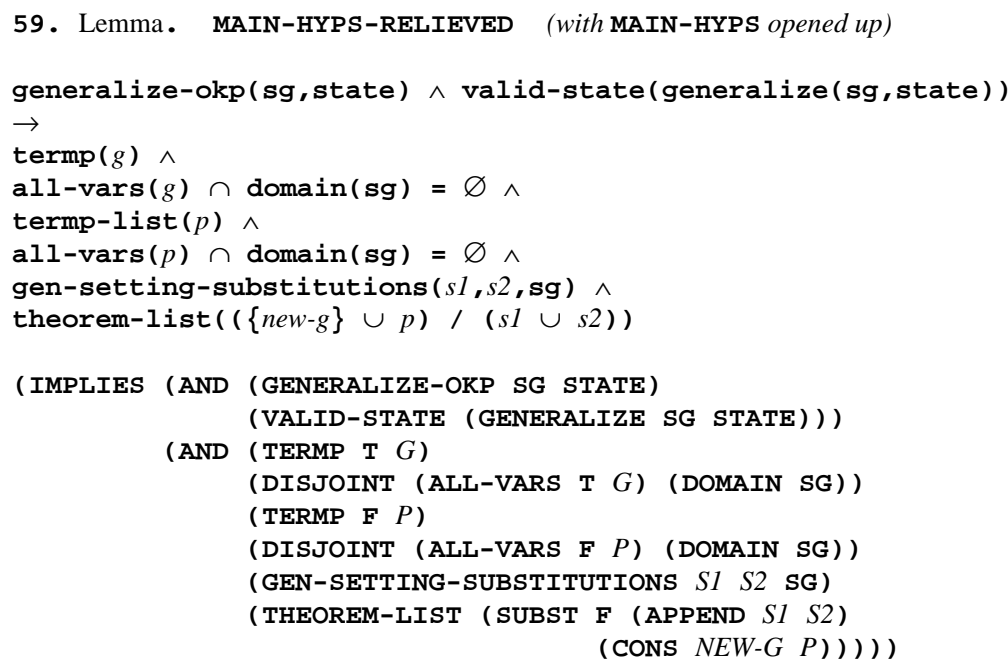

We thus have six cases to deal with. However, the first four are quite easy; the lemmas MAIN-HYPS-RELIEVED-n for $\mathbf{n}=1,2,3$, and 4 are events \#30 through \#33 in the file "generalize.events" (cf. Appendix A). It remains only to prove the other two cases, MAIN-HYPS-RELIEVED-5 and MAINHYPS-RELIEVED-6.

\subsubsection{Proof of the lemma MAIN-HYPS-RELIEVED-5}

Let us first state the lemma MAIN-HYPS-RELIEVED-5.

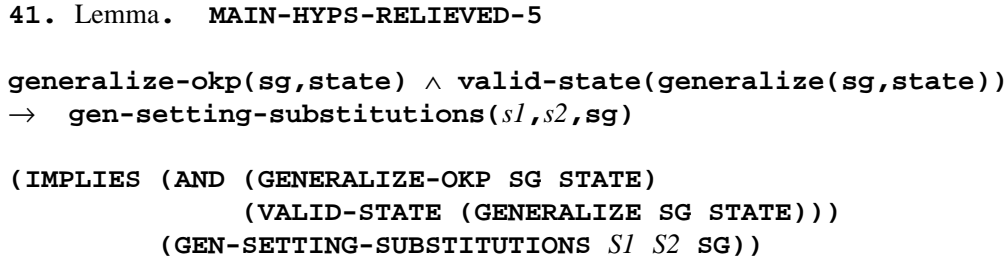

Opening up the function GEN-SETTING-SUBSTITUTIONS gives us a number of subgoals. The lemmas which follow cover all of these subgoals. Many of them are more general than the corresponding cases of the lemma MAIN-HYPS-RELIEVED-5. For example, in the first lemma below notice that domain-1 is arbitrary in place of the substitution denoted by the abbreviation domain-1. Generality often makes the theorem prover's job easier. 


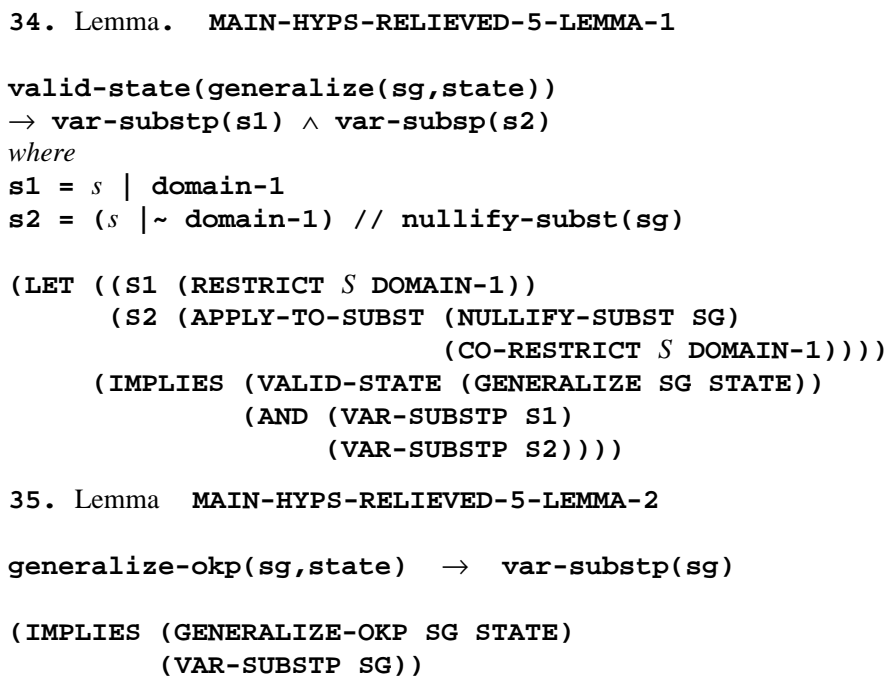

For the next two cases from the definition of GEN-SETTING-SUBSTITUTIONS, we first observe that the domain of the witnessing substitution $s$ is disjoint from the domain of $\mathbf{s g}$. This is an easy consequence of the definitions, which imply that domain (s) $\subseteq 2^{\text {nd }}$ (generalize (sg, state) $) \subseteq$ free and that free is disjoint from the domain of $\mathbf{s g}$.

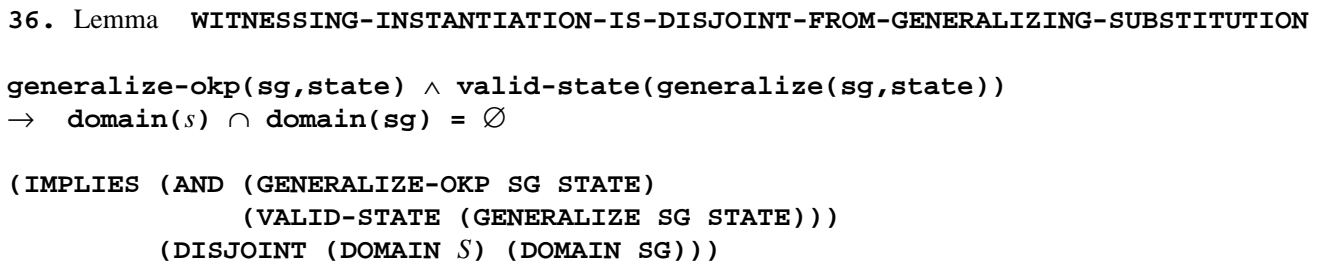

Since the domain of any restriction or co-restriction of a substitution is a subset of the original domain, and since the application of a substitution $\mathbf{s}$ to a substitution $\mathbf{s}^{\prime}$ has the same domain as does $\mathbf{s}^{\prime}$ (see DOMAINAPPLY-TO-SUBST in Section 3), the next two cases follow from the lemma displayed just above.

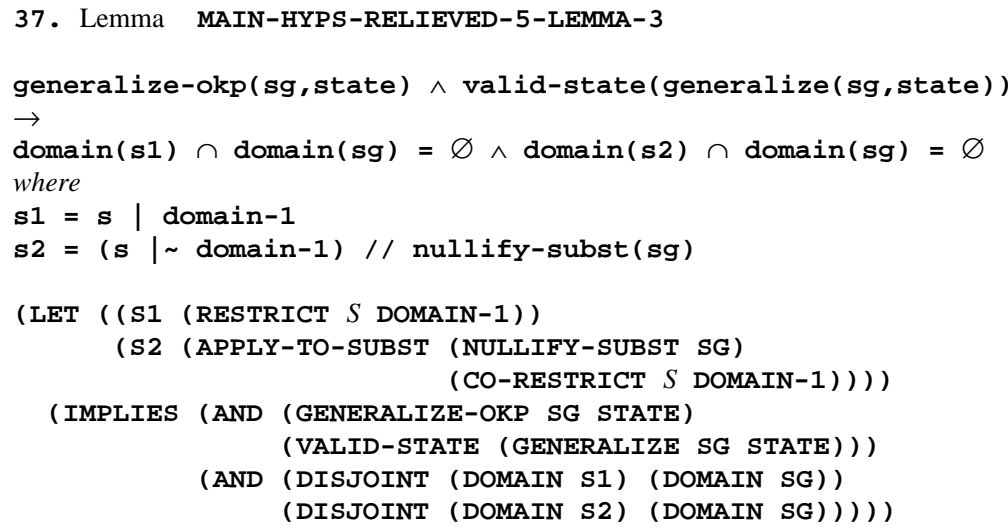

The following lemma MAIN-HYPS-RELIEVED-5-LEMMA-4 handles the next case. 


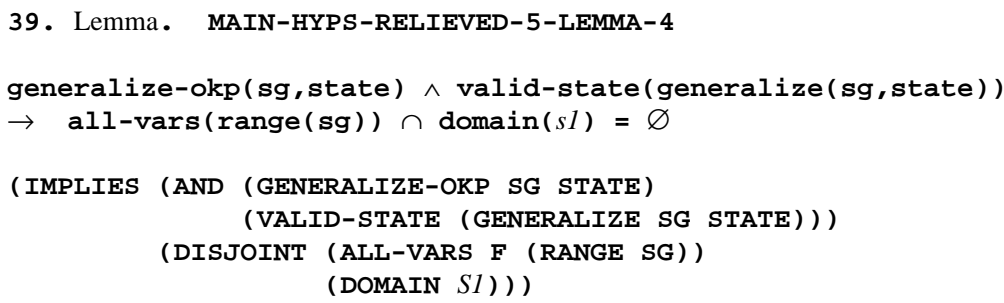

Let us argue informally for the correctness of this lemma. Assuming its hypotheses, we have:

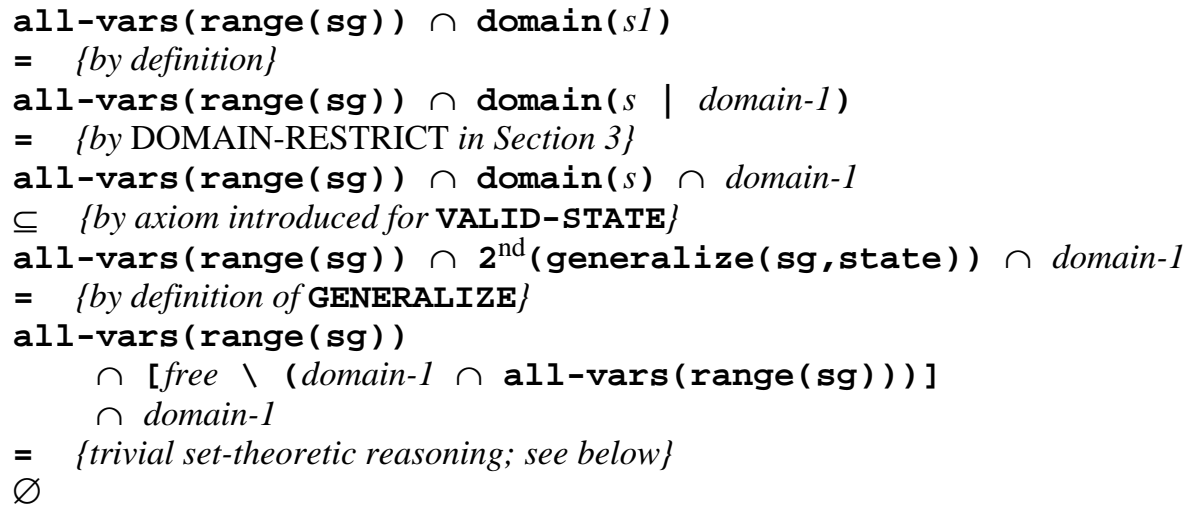

How would a person reason in the last step? A natural course would be to consider an arbitrary $\mathbf{x}$ and show that it if it belongs to all-vars (range (sg)) and also to [free $\backslash$ (domain- $1 \cap$ all-vars (range (sg) ) ) ], then it does not belong to domain-1. In fact the analogous fact is proved as a lemma for the intersection displayed two steps earlier in the informal proof above.

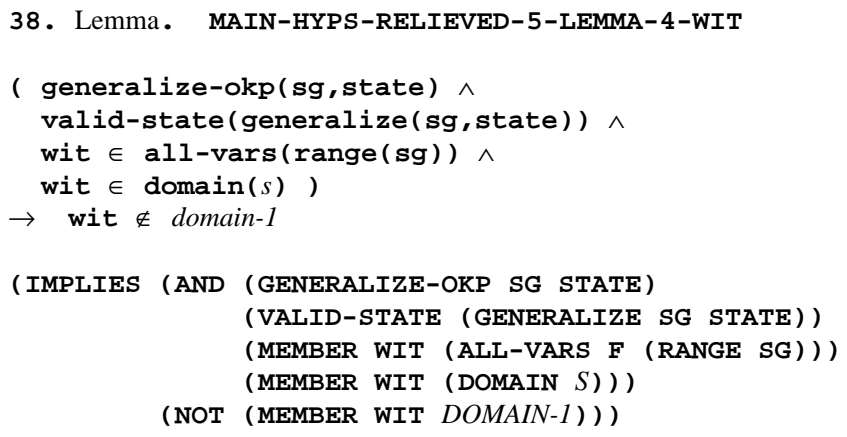

We have one final technical comment on the proof of MAIN-HYPS-RELIEVED-5-LEMMA-4. In addition to proving the lemma MAIN-HYPS-RELIEVED-5-LEMMA-4-WIT first (as a rewrite rule), a hint is also given to enable the lemma DISJOINT-WIT-WITNESSES. That lemma has the effect of reducing the statement that all-vars (range (sg)) is disjoint from the domain of $s l$ to the question issue of whether a particular value could belong to both of them. For a description of that lemma, see Section 3.

The final case goes through automatically, though here it is crucial that $s 2$ is built using 
NULLIFY-SUBST; see the lemma DISJOINT-ALL-VARS-RANGE-APPLY-SUBST-NULLIFY-SUBST in Section 3.

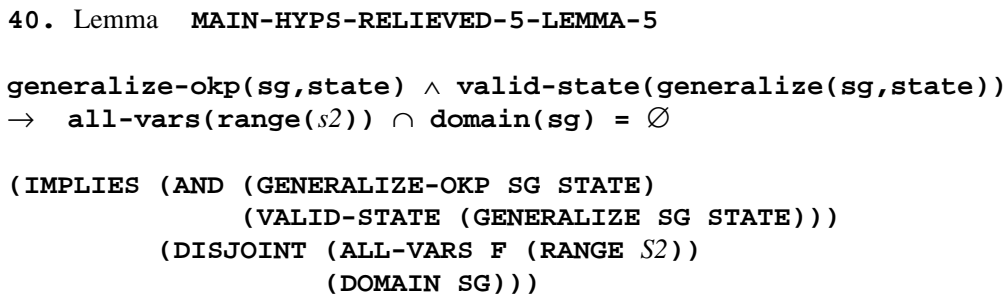

\subsubsection{Proof of the lemma MAIN-HYPS-RELIEVED-6}

Now all that is left is the proof of the lemma MAIN-HYPS-RELIEVED-6. Here is its statement.

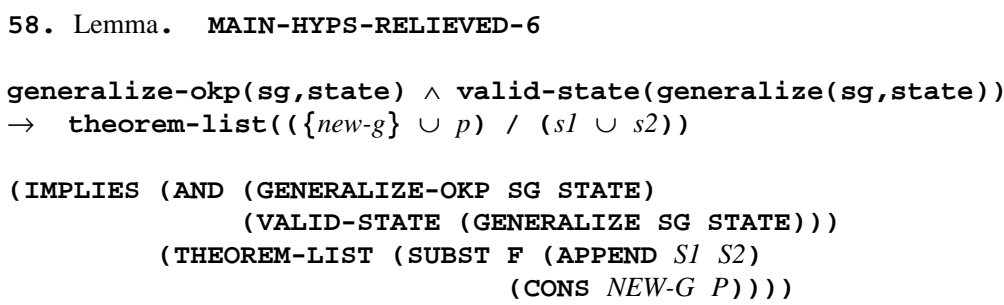

Here is a very high-level view of the proof, which incidentally should show why we chose to bring in the notion of "gen-closure" Because of the way that the function GEN-CLOSURE is defined (event \#10 above), the set domain- 1 has the following property: for every goal $\mathbf{x}$ in the new state generalize (sg, state), the set of free variables in that state that occur in $\mathbf{x}$ are either contained in domain- 1 or are disjoint from it. In the former case, which includes the case $\mathbf{x}=n e w-g$, no variable occurring in $\mathbf{x}$ is in the domain of $s 2$, and it follows

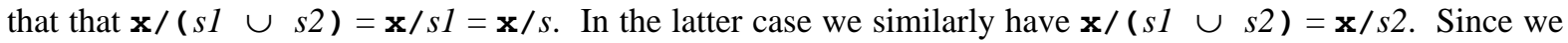
have already dealt with the case $\mathbf{x}=n e w-g$, we may assume that $\mathbf{x} \in \mathbf{p}$, and by a little additional technical argument we can show that $\mathbf{x} / s 2$ is an instance of $\mathbf{x} / s$. So we have that $\mathbf{x} /(s 1 \cup s 2)$ is an instance of $\mathbf{x} / s$, and since $\mathbf{x} / s$ is a theorem (by definition of $s$ and the VALID-STATE hypothesis), so is $\mathbf{x} /\left(\begin{array}{lll}(s 1 & \cup\end{array}\right)$.

Let us proceed now along the lines of the mechanically-checked proof. By opening up SUBST and THEOREM-LIST we can break MAIN-HYPS-RELIEVED-6 into the following two goals.

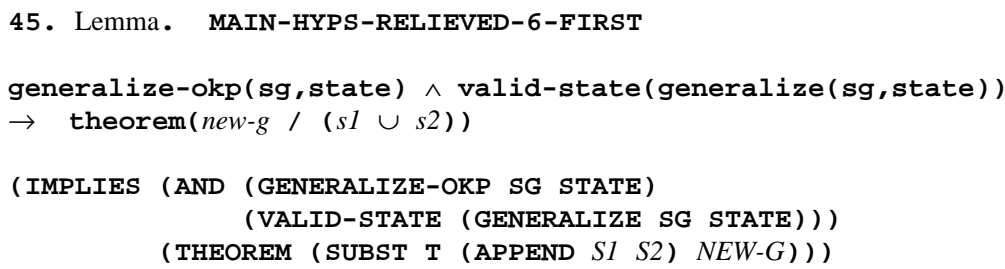




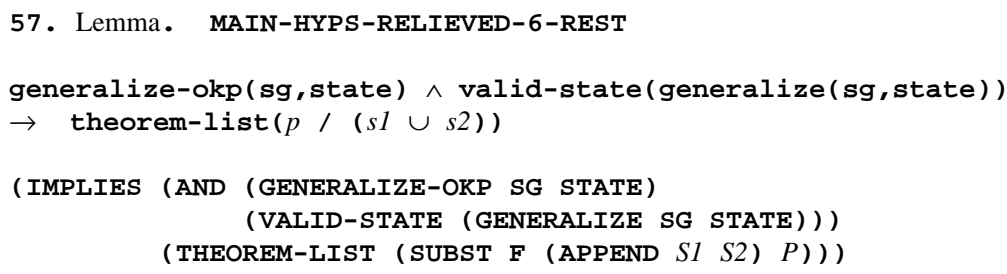

Let us consider these in turn.

5.3.2(1) Proof of the lemma MAIN-HYPS-RELIEVED-6-FIRST. Here is an informal proof of the first of these two lemmas. We begin with a key observation, which we will both prove and use presently.

$$
\text { domain }(s) \cap \text { all-vars }(\text { new-g) } \subseteq \text { domain- } 1
$$

Now recall the lemma SUBST-APPEND-NOT-OCCUR-2 (stated in Subsection 5.2 above) which says if no variable of a term $\mathbf{x}$ belongs to the domain of a substitution $\mathbf{s} 2$ then $\mathbf{x} /(\mathbf{s} 1 \cup \mathbf{s} 2)=\mathbf{x} / \mathbf{s} 1$. The domain of $s 2$ is equal to domain(s) \ domain-1; hence the requirement for SUBST-APPEND-NOTOCCUR-2 that domain $(s 2)$ be disjoint from all-vars $(n e w-g)$ holds by $(*){ }^{8}$ So assuming our hypotheses of generalize-okp(sg, state) and valid-state(generalize(sg,state)), we may summarize the argument as follows.

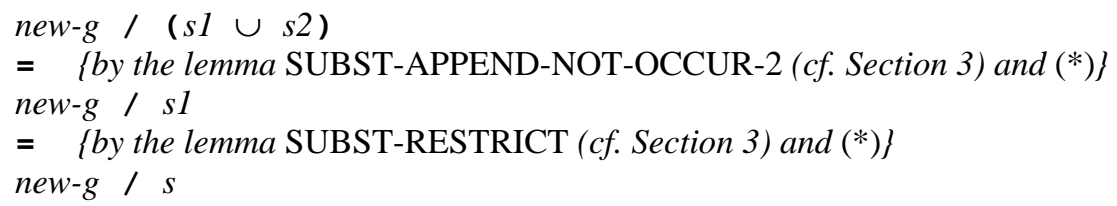

It remains to check that $(*)$ holds. Consider the following lemma.

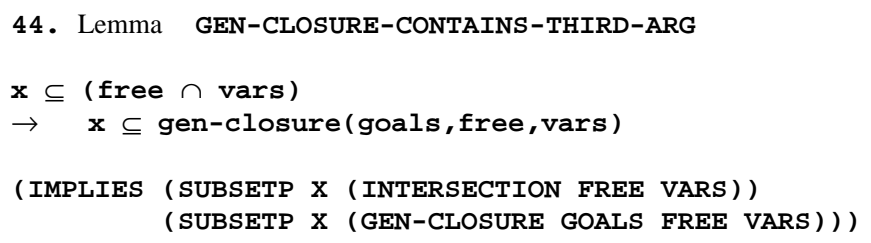

If we apply this lemma with goals $:=\{$ new- $g\} \cup p$, free $=$ free, vars $=$ all-vars $(n e w-g)$, and $\mathbf{x}:=$ domain $(s) \cap$ all-vars $($ new- $g$ ), the resulting instance can be expressed using our abbreviations as follows.

\footnotetext{
${ }^{8}$ The lemmas used in this argument are DOMAIN-CO-RESTRICT from "alists.events" and DISJOINT-SET-DIFF-GENERAL from "sets.events"
} 


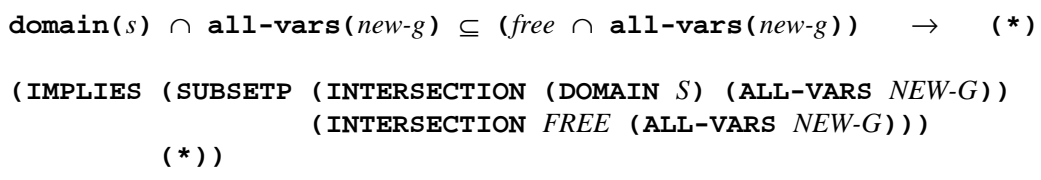

So in order to prove $\left(^{*}\right)$, it suffices to prove the hypothesis of this implication, which in turn follows from

$\operatorname{domain}(s) \subseteq 2^{\text {nd }}($ generalize $(\mathbf{s g}$, state $)) \subseteq$ free

The first inclusion follows from the fact that the domain of $s$ is contained in the free variables of the new (generalized) state, which is part of the VALID-STATE hypothesis. The second inclusion is just the lemma SUBSETP-CDR-GENERALIZE from Subsection 5.1 above. This concludes the proof of MAIN-HYPSRELIEVED-6-FIRST.

In fact we close with one technical comment. The lemma CAR-GENERALIZE is proved before the lemma GEN-CLOSURE-CONTAINS-THIRD-ARG above so as to speed up the proofs. The idea is that we only want to invoke the rather hairy definition of GENERALIZE when we are looking at goals, not when we are simply asking about the witnessing substitution.

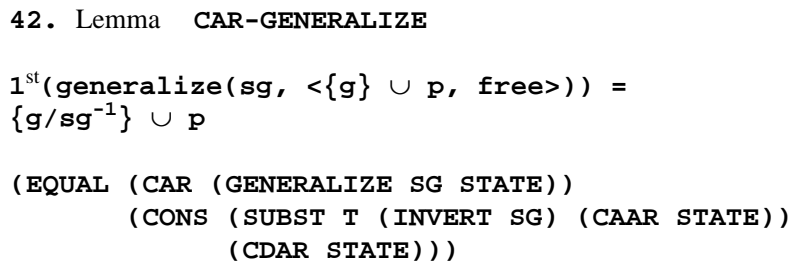

5.3.2(2) Proof of the lemma MAIN-HYPS-RELIEVED-6-REST. We now move to the proof of our final goal, which once again is:

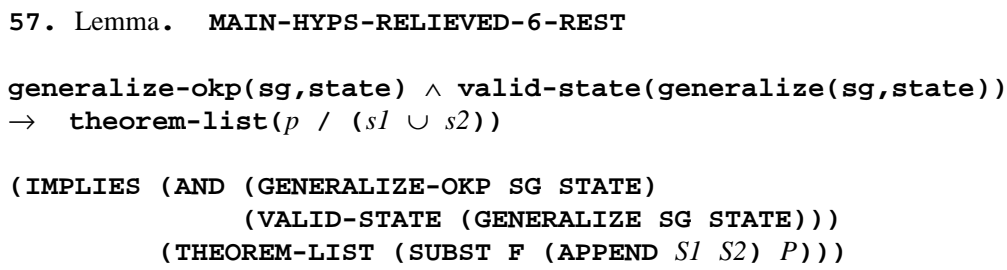

Let us begin with the following key notion suggested by the informal proof given above. It asserts that every goals's free variables are either contained in the set $\mathbf{x}$ or are disjoint from $\mathbf{x}$. 


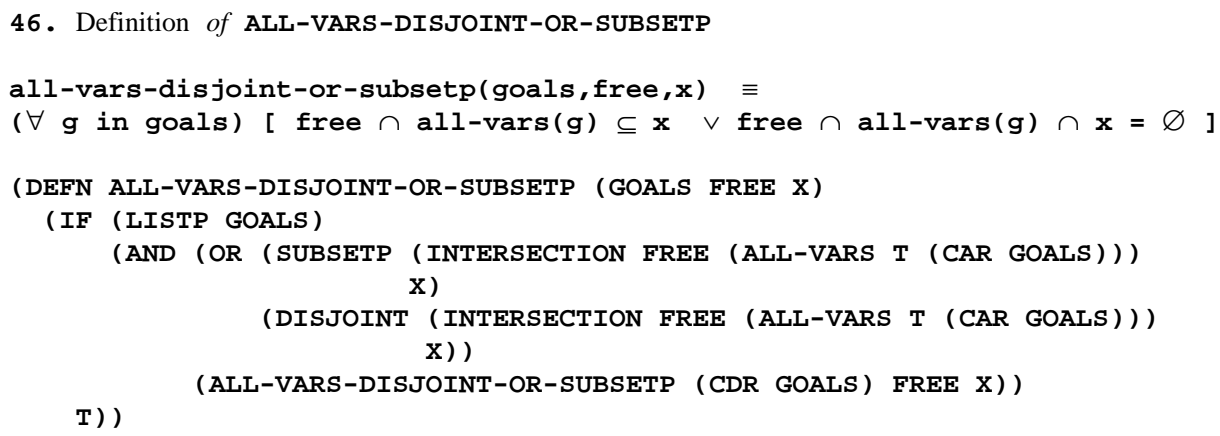

Observe that the set of goals $p$ has the above property with respect to the free variables of the generalized state and the appropriate "'gen-closure', domain-1:

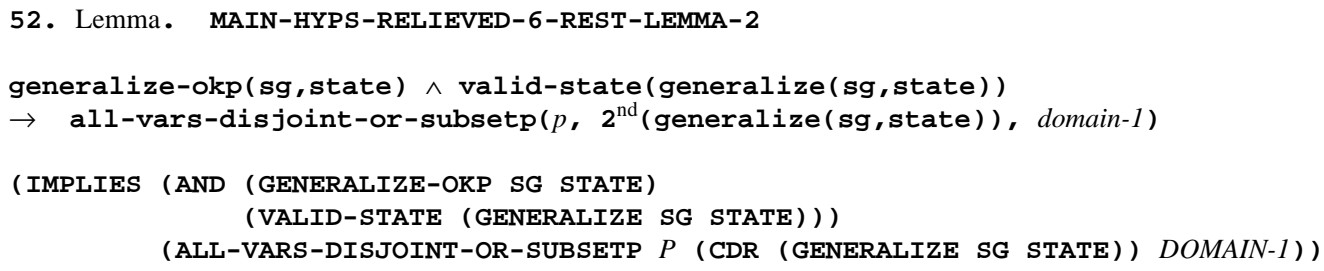

This follows from the definition of domain- 1 and the following observation. Actually, the following lemma relevant in the special case (instance) where new-free is $\mathbf{2}^{\text {nd }}$ (generalize (sg, state) ), i.e. the set of free variables of the new (generalized) state; free is free; goals is $p ; \mathbf{g}$ is $g$; and vars is domain- 1 .

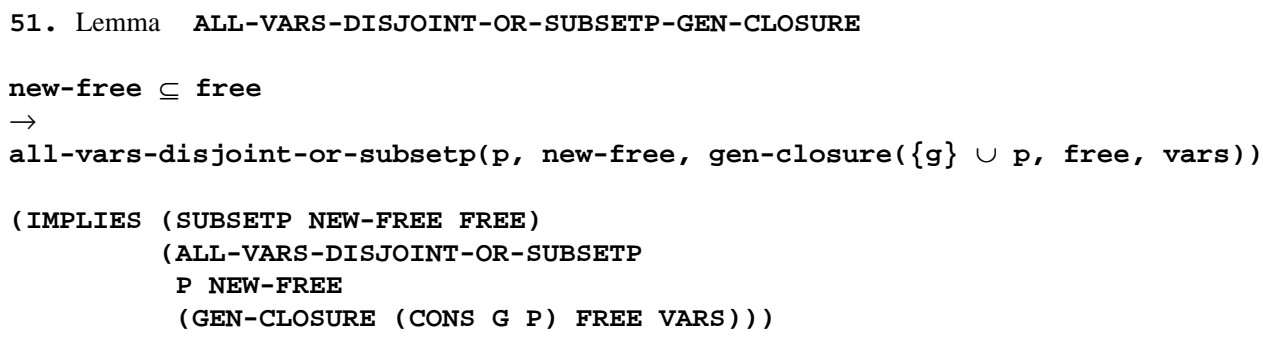

Let us attempt to finish the proof of our remaining goal MAIN-HYPS-RELIEVED-6-REST with informal reasoning. Assume its hypotheses. Let $\mathbf{x}$ be any goal in $p$; then by the VALID-STATE hypothesis, we have theorem (x). Moreover, the lemma MAIN-HYPS-RELIEVED-6-REST-LEMMA-2 above implies says that the set of free variables occurring in $\mathbf{x}$ is contained in or disjoint from domain-1. Hence there are two cases. We follow the outline given at the start of this subsection 5.3.2 (just below the statement of the lemma MAIN-HYPS-RELIEVED-6).

Case 1: all-vars $(\mathbf{x}) \subseteq$ domain-1. Then since domain $(s 2)=\operatorname{domain}(s) \backslash$ domain-1 (by definition of $s 2$ and the lemmas DOMAIN-CORESTRICT and DOMAIN-APPLY-TO-SUBST in Section 3), it follows from the case hypothesis that 
$\operatorname{all-vars}(\mathbf{x}) \cap \operatorname{domain}(s 2)=\varnothing$.

Therefore

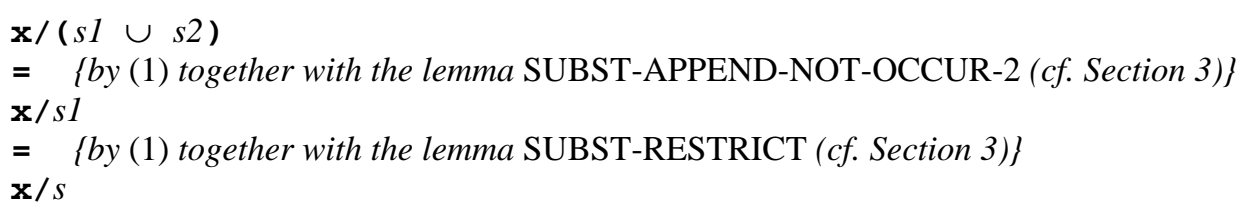

Case 2: all-vars (x) $\cap$ domain-1 $=\varnothing$. The argument is a little more involved in this case, because $s 2$ is not simply a co-restriction of $s$. Recall that $s 2$ is defined as ( $s \quad \mid \sim$ domain- 1 ) // nullify-subst (sg). This time we argue as follows.

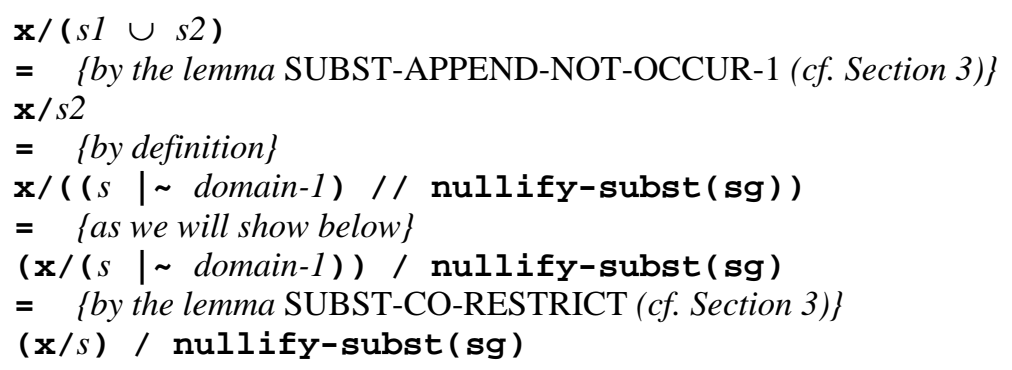

Therefore $\mathbf{x} /(s 1 \cup s 2)$ is a theorem (cf. event \#54 in "generalize.events" in the Appendix, which we omit here), since it is an instance of $\mathbf{x} / s$ (which is a theorem by the VALID-STATE hypothesis). But it remains to explain the reason "as we will show below" for the penultimate step above. The following lemma is the key. It is applied automatically by the theorem prover's rewriter using the substitution $\mathbf{\text { sg }}:=$ nullify-

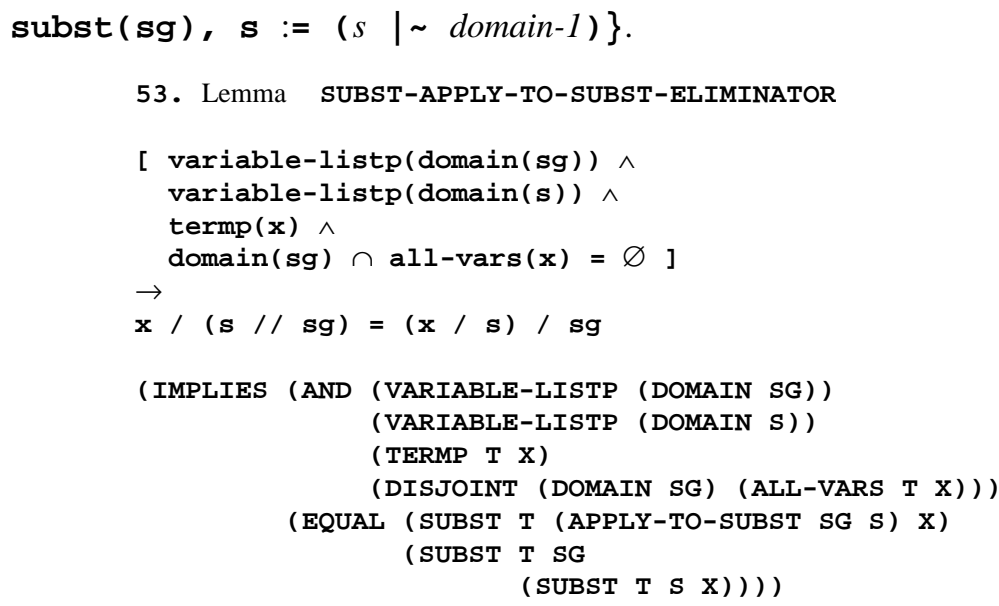

Notice that by the lemma DOMAIN-NULLIFY-SUBST (cf. Section 3), we can safely equate the domains of sg and nullify-subst (sg). But why can we assume that the domain of $\mathbf{s g}$ is disjoint from the variables of $\mathbf{x}$ ? Recall that $\mathbf{x}$ is an arbitrary member of the set of goals from $p$ that (by the Case 2 hypothesis) do not 
intersect domain-1. That is, the following lemma should suffice to conclude the proof. (The definition of the function GOALS-DISJOINT-FROM-VARS will follow.)

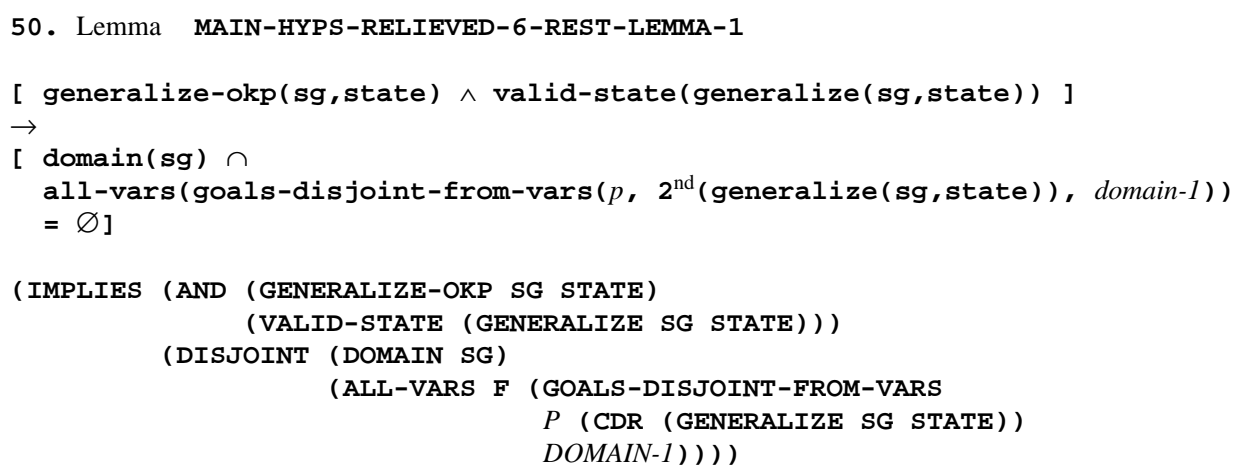

And here is the obvious definition of GOALS-DISJOINT-FROM-VARS, followed by an important property of this function.

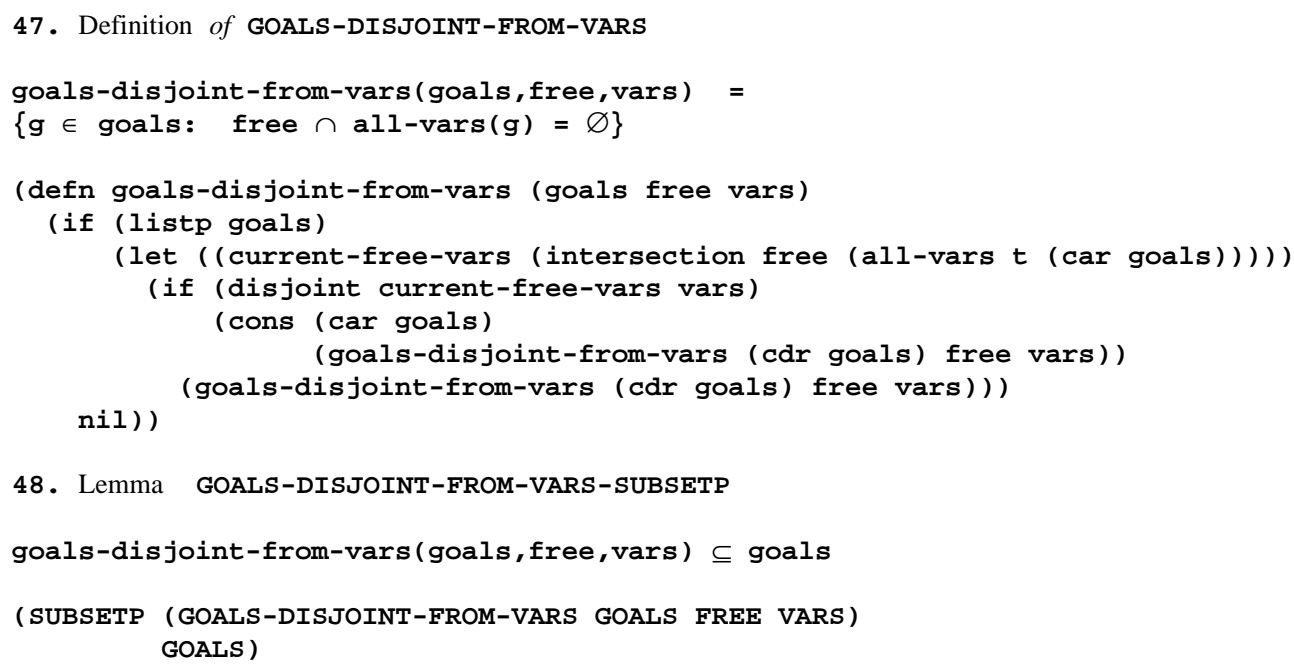

Event \#49, DISJOINT-ALL-VARS-GOALS-DISJOINT-FROM-VARS, is merely a technical lemma that is necessary because of the theorem prover's difficulty in relieving hypotheses of rewrite rules that contain variables not bound in the conclusion. We omit its statement here.

We conclude by summarizing the top-level structure of the proof of the lemma MAIN-HYPSRELIEVED-6-REST, which is motivated by the discussion above. This lemma is an immediate consequence of the following lemma, in conjunction with the lemma MAIN-HYPS-RELIEVED-6-REST-LEMMA-1 (event \#50) and MAIN-HYPS-RELIEVED-6-REST-LEMMA-2 (event \#52), already explained above, which are used to relieve its last two hypotheses. Notice that this lemma is somewhat more abstract than those two, in that it refers to arbitrary values of $\mathbf{p}, \mathbf{s}$, domain-1, and new-free.

56. Lemma MAIN-HYPS-RELIEVED-6-REST-GENERALIZATION 


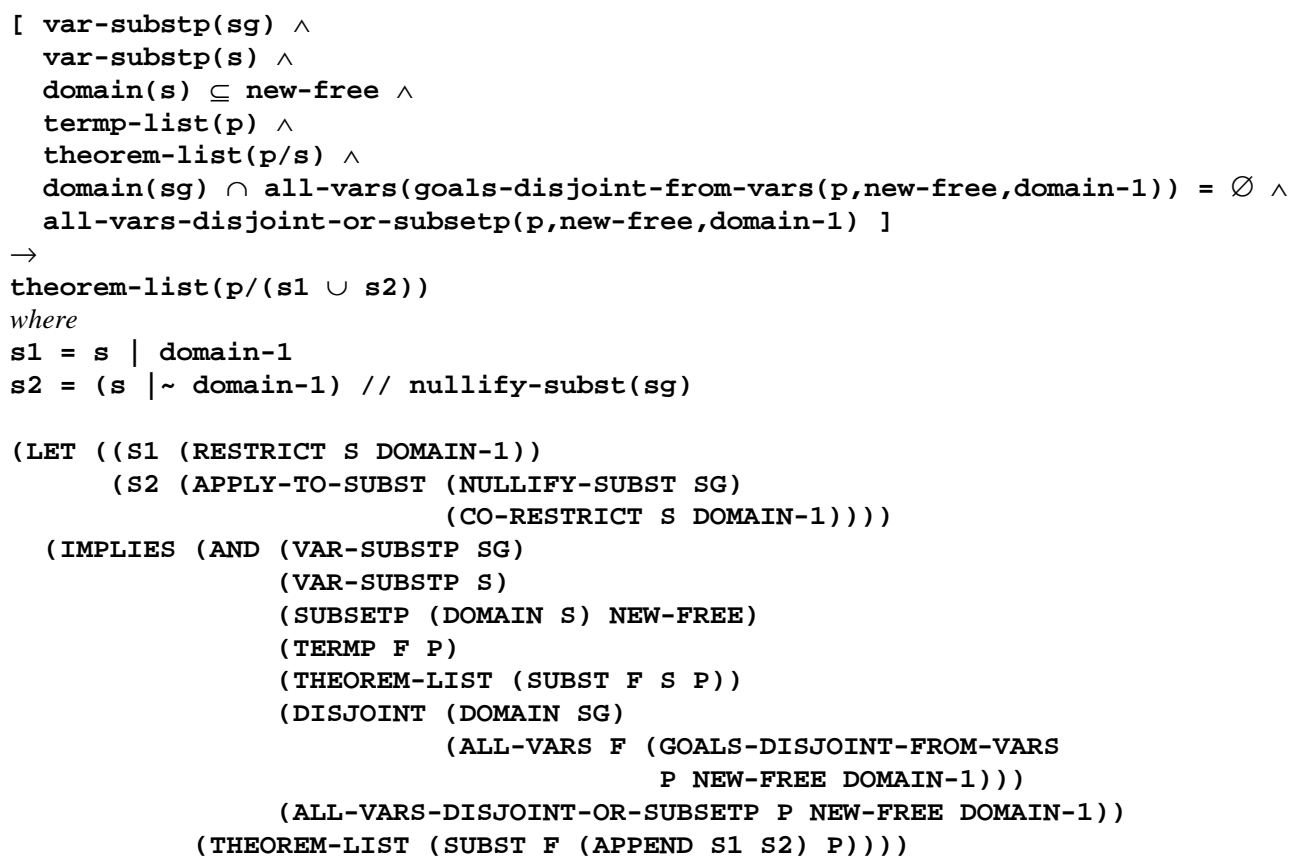

The theorem prover implements informal arguments presented above when proving this theorem by induction on the length of $\mathbf{p}$. However, we encountered difficulties at first in finding the right argument, at least during our second proof effort (see Subsection 1.2). The remainder of this section contains an edited version of the comments made during that proof, just after completion of MAIN-HYPS-RELIEVED-6-REST-LEMMA-2 (so that all that was left was the proof of MAIN-HYPS-RELIEVED-6-REST-GENERALIZATION). All of this below may be safely omitted; it's there simply for those familiar with the Boyer-Moore theorem prover who want to dig a little deeper into the details of the proof effort.

5.3.2(3) Some comments on the proof of the lemma MAIN-HYPS-RELIEVED-6-REST-GENERALIZATION. Finally, all that's left is MAIN-HYPSRELIEVED-6-REST-GENERALIZATION. An attempted proof by induction of that theorem results in 11 goals, all but one of which goes through automatically. The remaining one is as follows. 


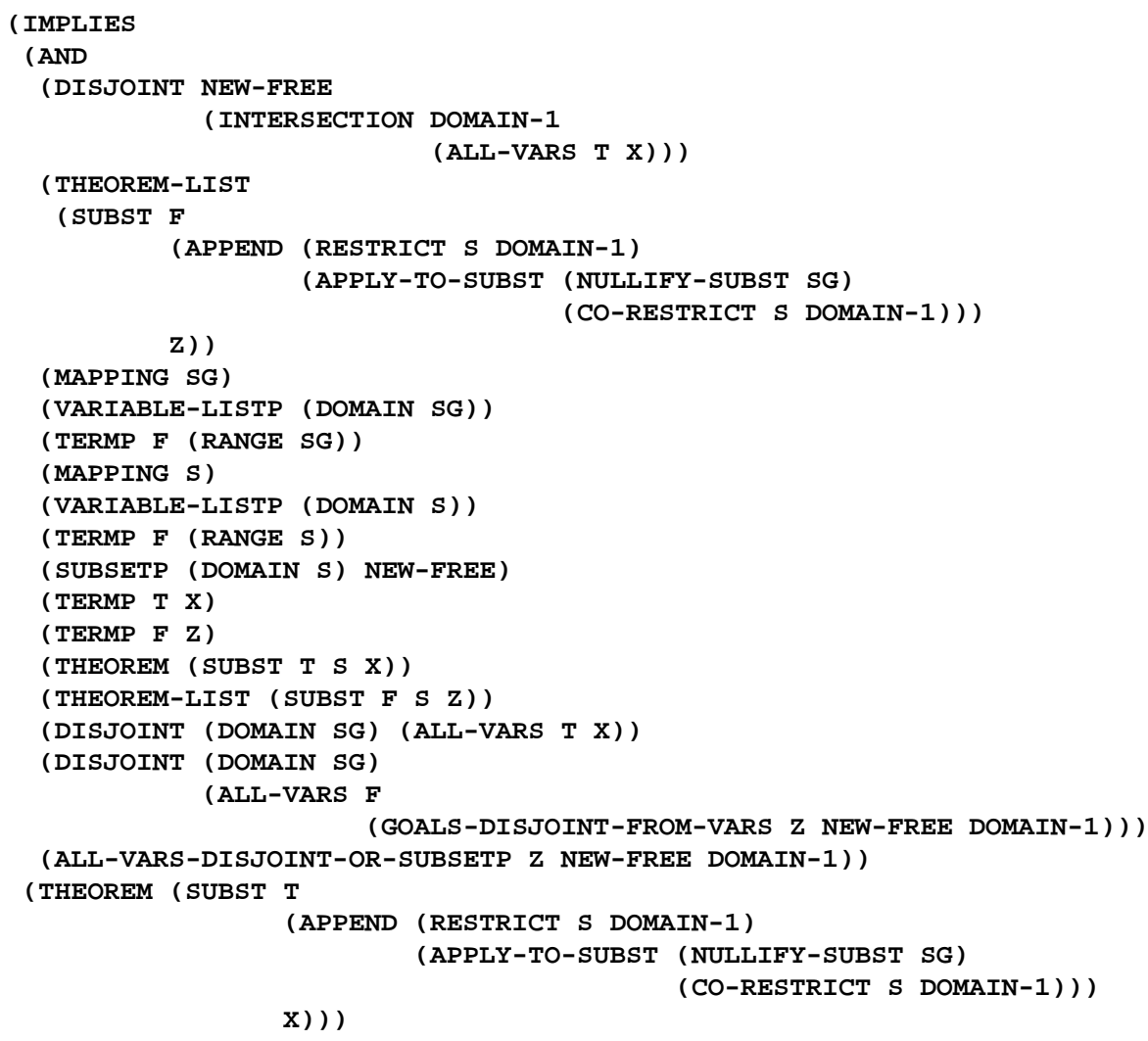

x) ))

Let us attempt to prove this goal with PC-NQTHM, thus seeing why the rewriter can't handle it automatically. With the aid of PC-NQTHM's SHOW-REWRITES command, we see that we would like to rewrite with the lemma SUBST-APPEND-NOT-OCCUR-1 (see Section 3) to replace the conclusion with:

(THEOREM (SUBST T

(APPLY-TO-SUBST (NULLIFY-SUBST SG)

X))

(CO-RESTRICT S DOMAIN-1))

However, in order to do that we see (using PC-NQTHM's REWRITE command) that we need to know that under the hypotheses, the following holds.

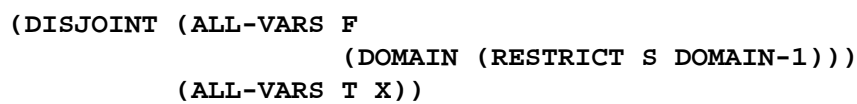

One would think that this follows quite clearly from just two of the hypotheses:

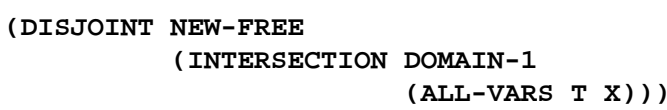

This is one of those cases of a problem with free variables in hypotheses that are so annoying. The lemma 
DOMAIN-RESTRICT has been proved in "alists.events" (see also Section 3) to help with this. But then we lose the effect of an existing lemma which applied directly to simplify the term (ALL-VARS F (DOMAIN (RESTRICT S DOMAIN-1))) (a familiar phenomenon for those familiar with Knuth-Bendix completion). The lemma VARIABLE-LISTP-INTERSECTION has since been proved in "terms.events" to take care of that problem.

Now it looks like the rewrite using SUBST-APPEND-NOT-OCCUR-1 should succeed, since all hypotheses are relieved by rewriting alone. Just to make sure, we back up in PC-NQTHM and see if the BASH command (which calls the Boyer-Moore prover's simplifier) uses this rule on our original goal. Sure enough, it does.

Having successfully applied PC-NQTHM's REWRITE command and relieved the resulting hypothesis, we now have a conclusion that is the one displayed above, i.e.

(THEOREM (SUBST T

(APPLY-TO-SUBST (NULLIFY-SUBST SG)

x) )

(CO-RESTRICT S DOMAIN-1))

Since (as we already know) (NULLIFY-SUBST SG) has the same domain as does SG, and since the hypotheses imply that (DOMAIN SG) is disjoint from the variables of $\mathbf{x}$, the SUBST expression in this conclusion should simplify to:

(SUBST T (NULLIFY-SUBST SG)

(SUBST T (CO-RESTRICT S DOMAIN-1)

$\mathrm{X))}$

We therefore need the lemma SUBST-APPLY-TO-SUBST-ELIMINATOR below (which is used under the substitution where S gets (CO-RESTRICT S DOMAIN-1) and SG gets (NULLIFY-SUBST SG)). [And so on.......] 


\section{Appendix A}

\section{Events Files: sets, alists, terms, and generalize}

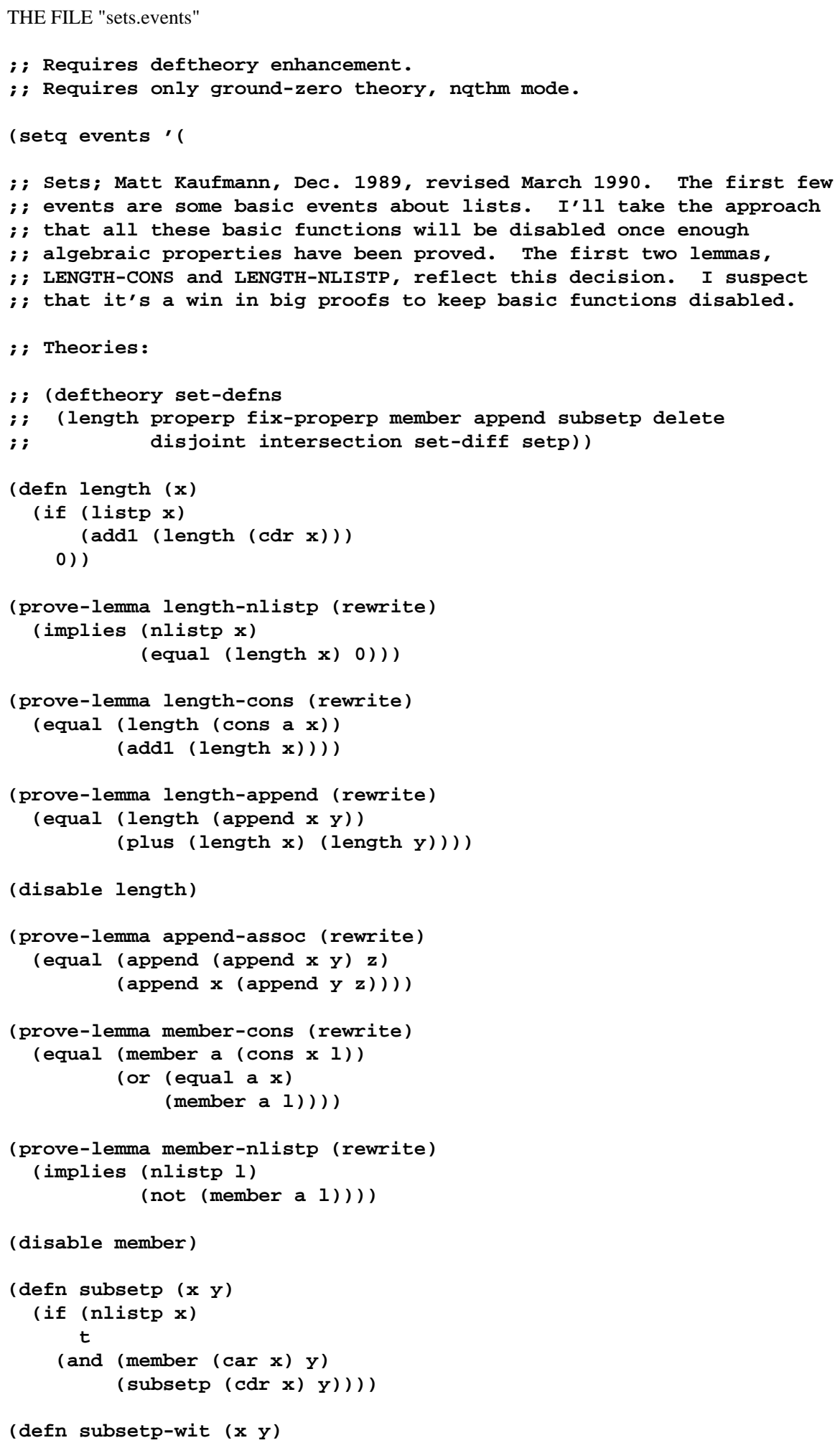




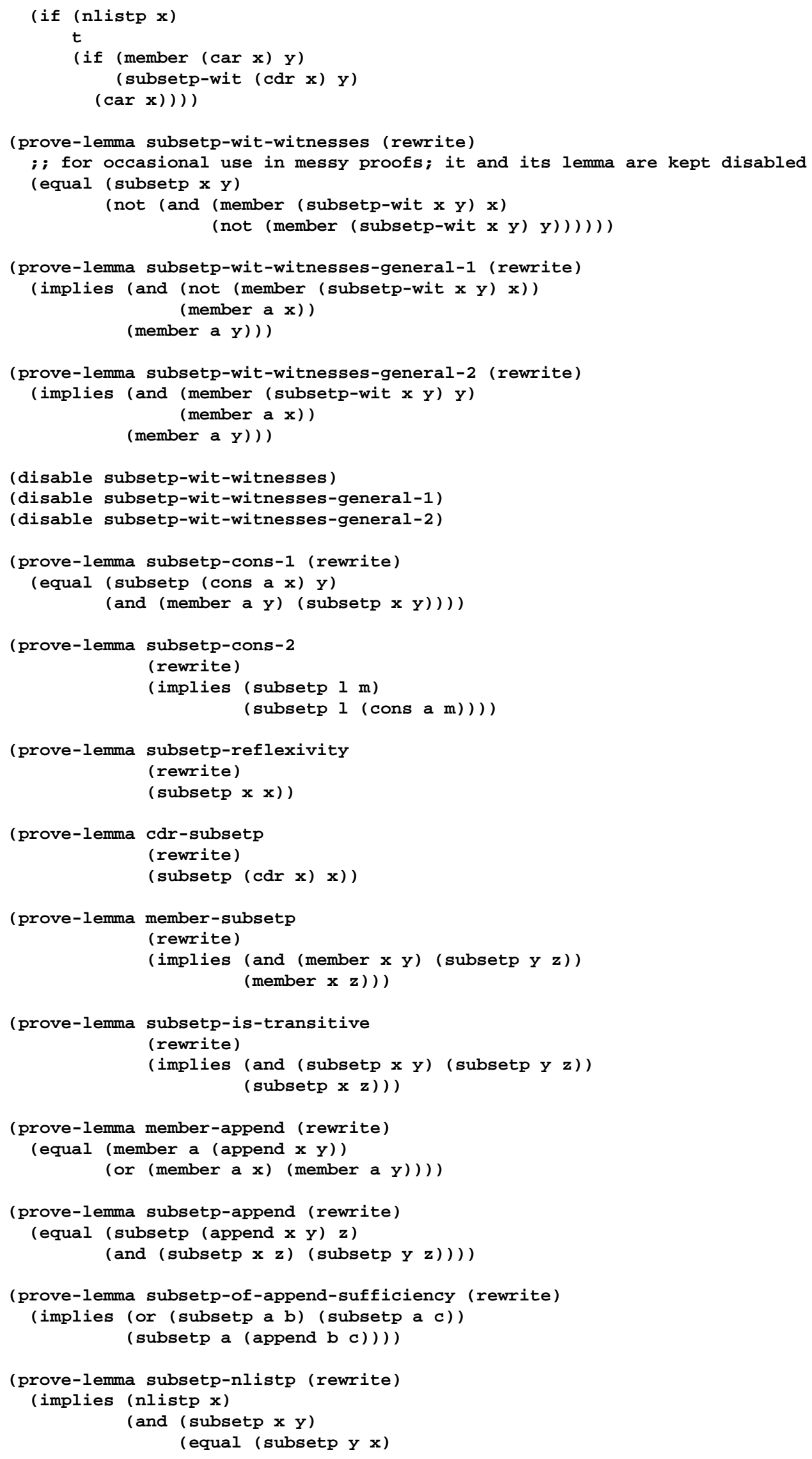


$($ nlistp y))) ))

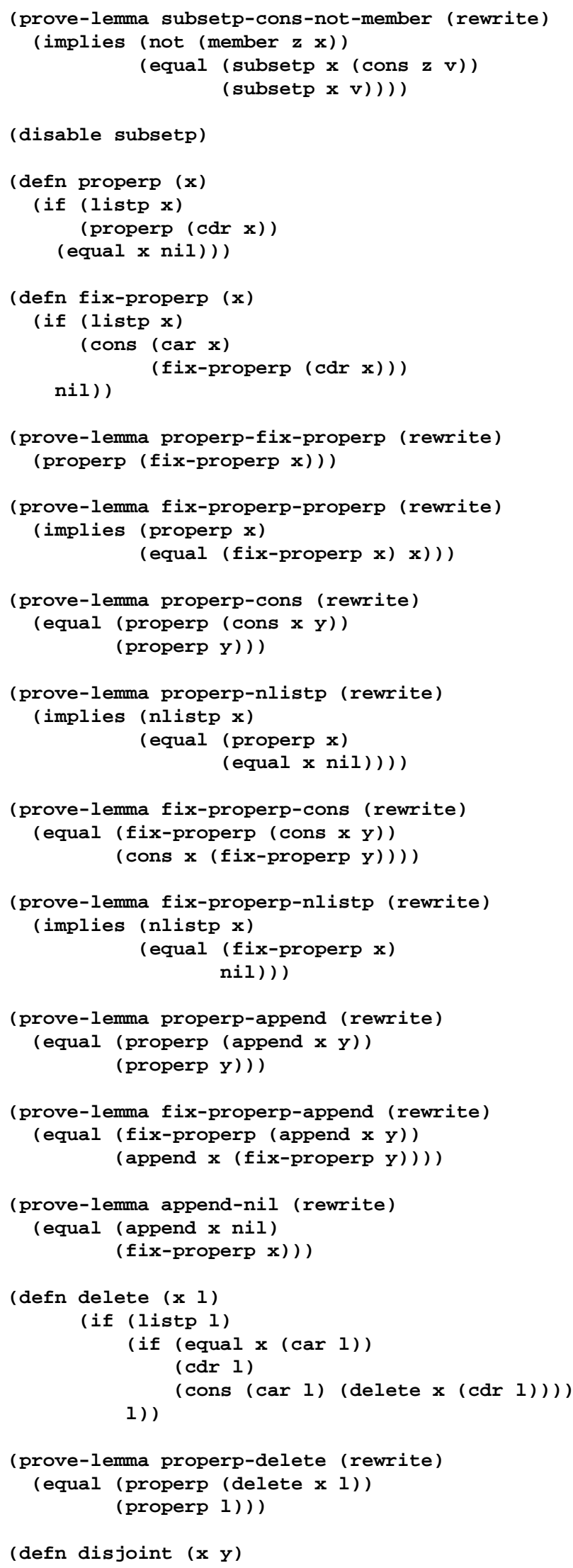




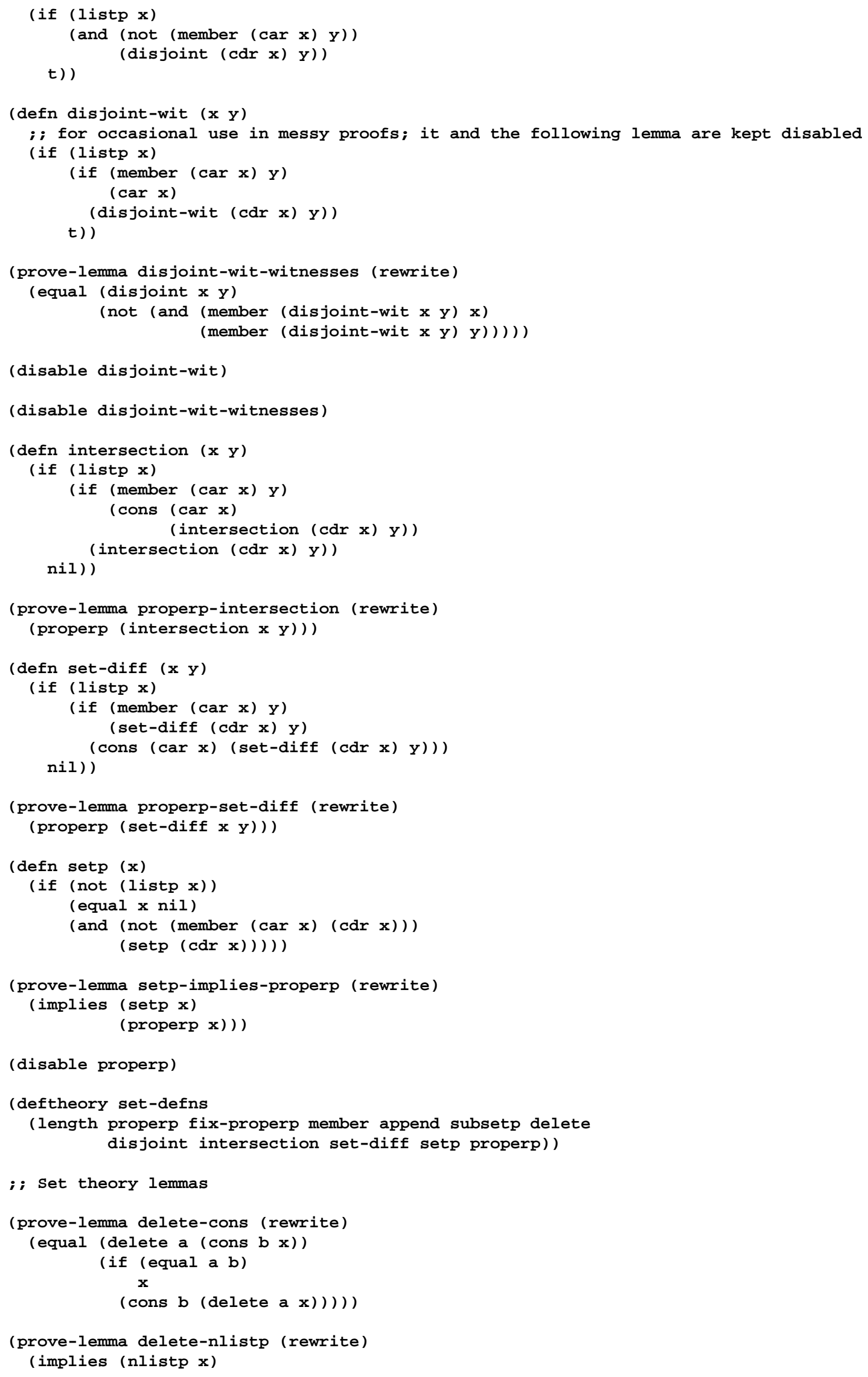




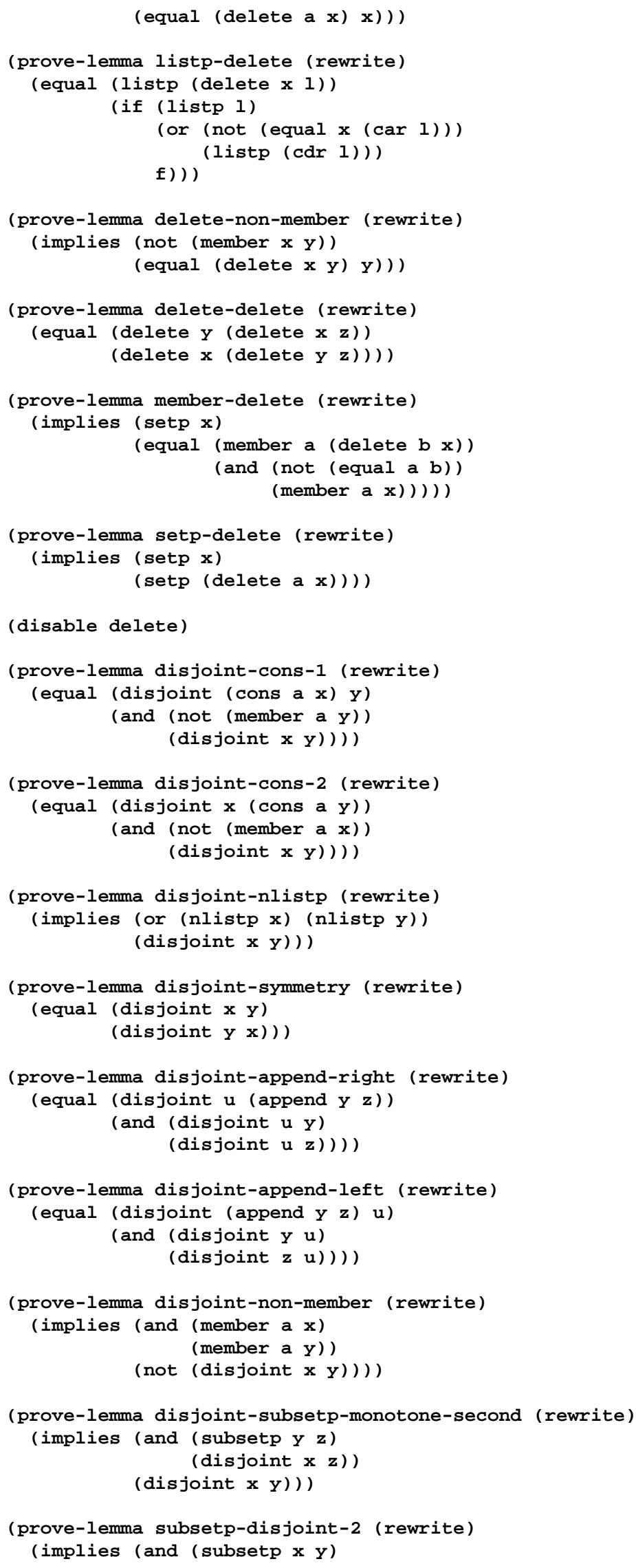




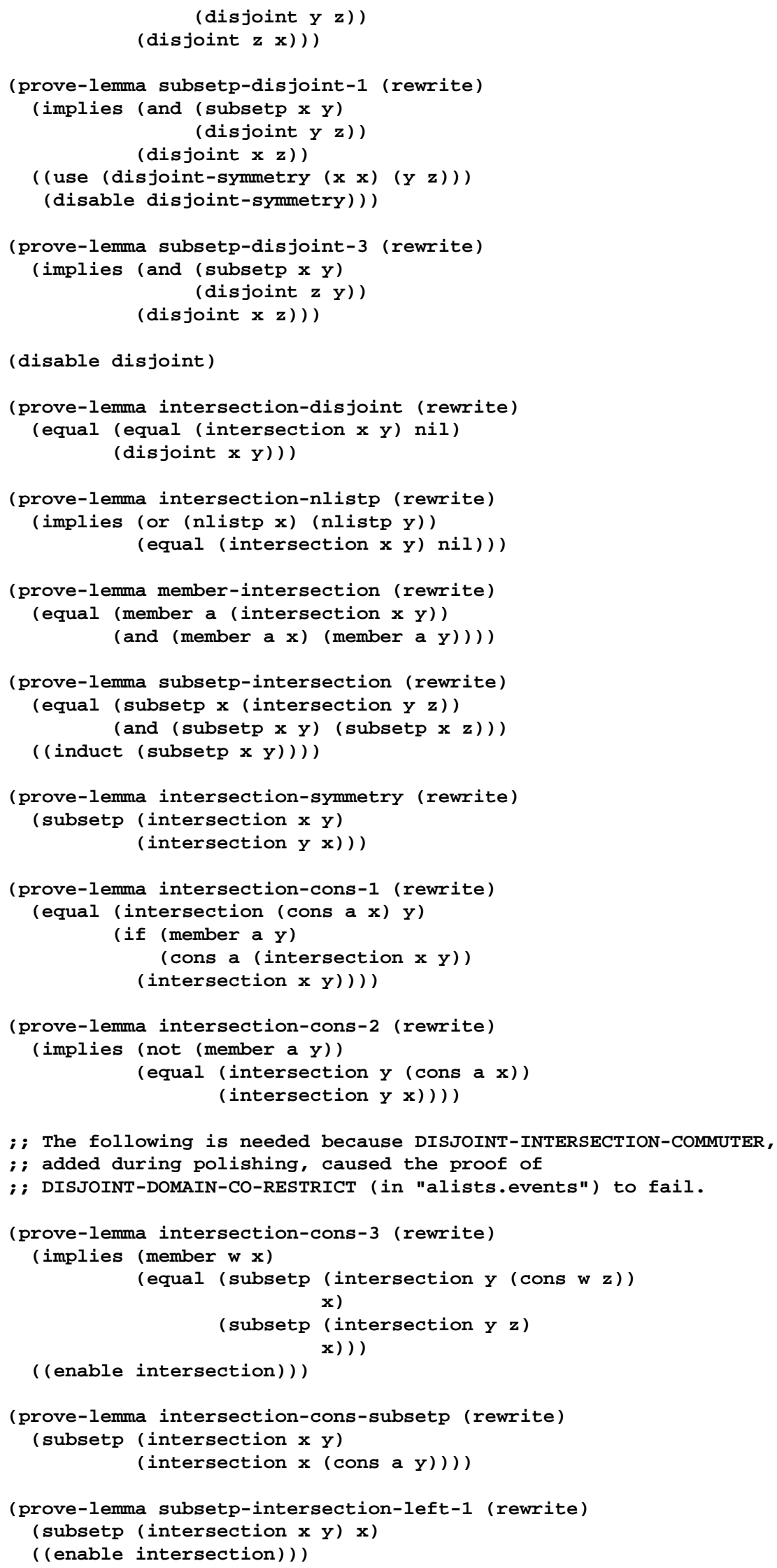




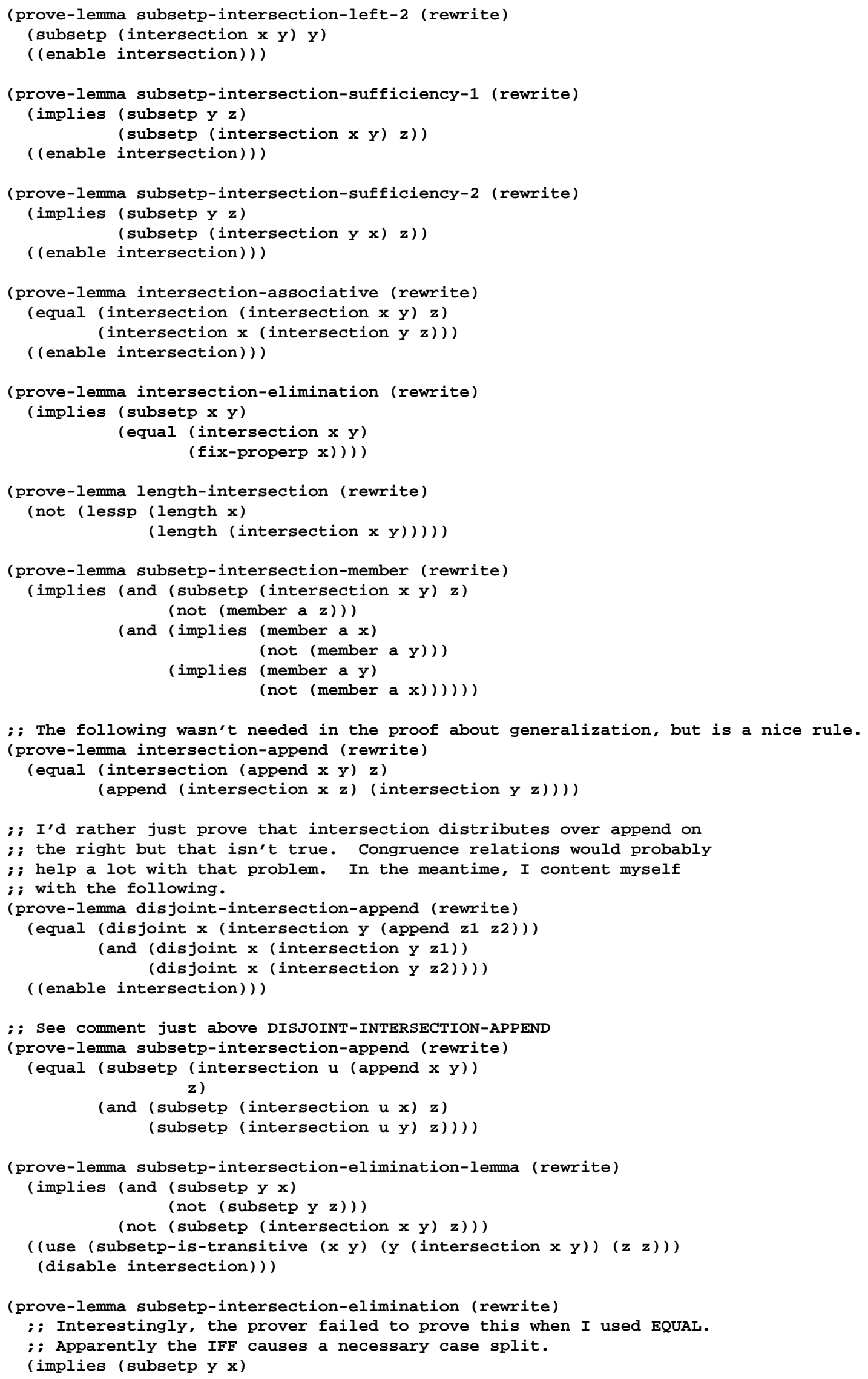




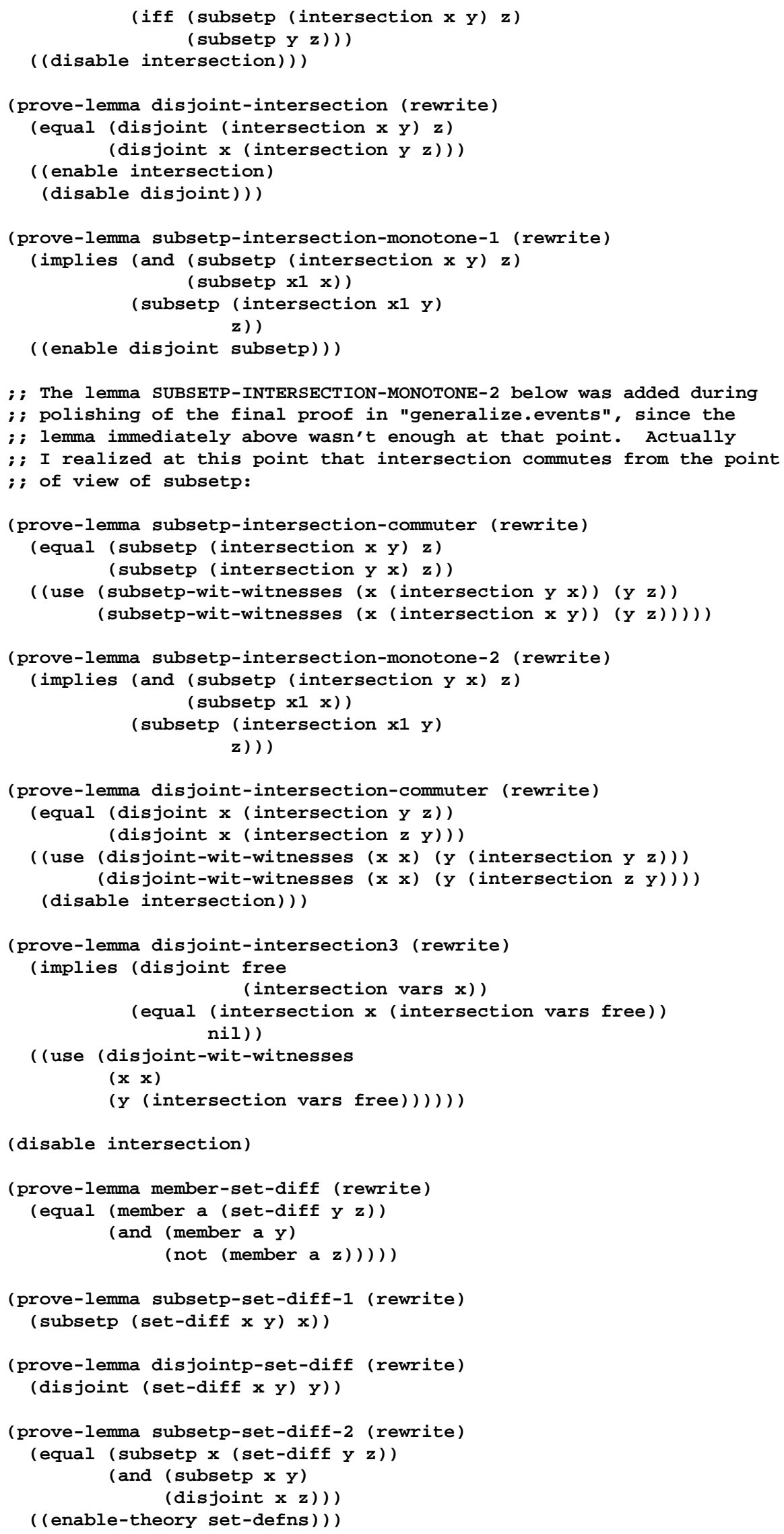




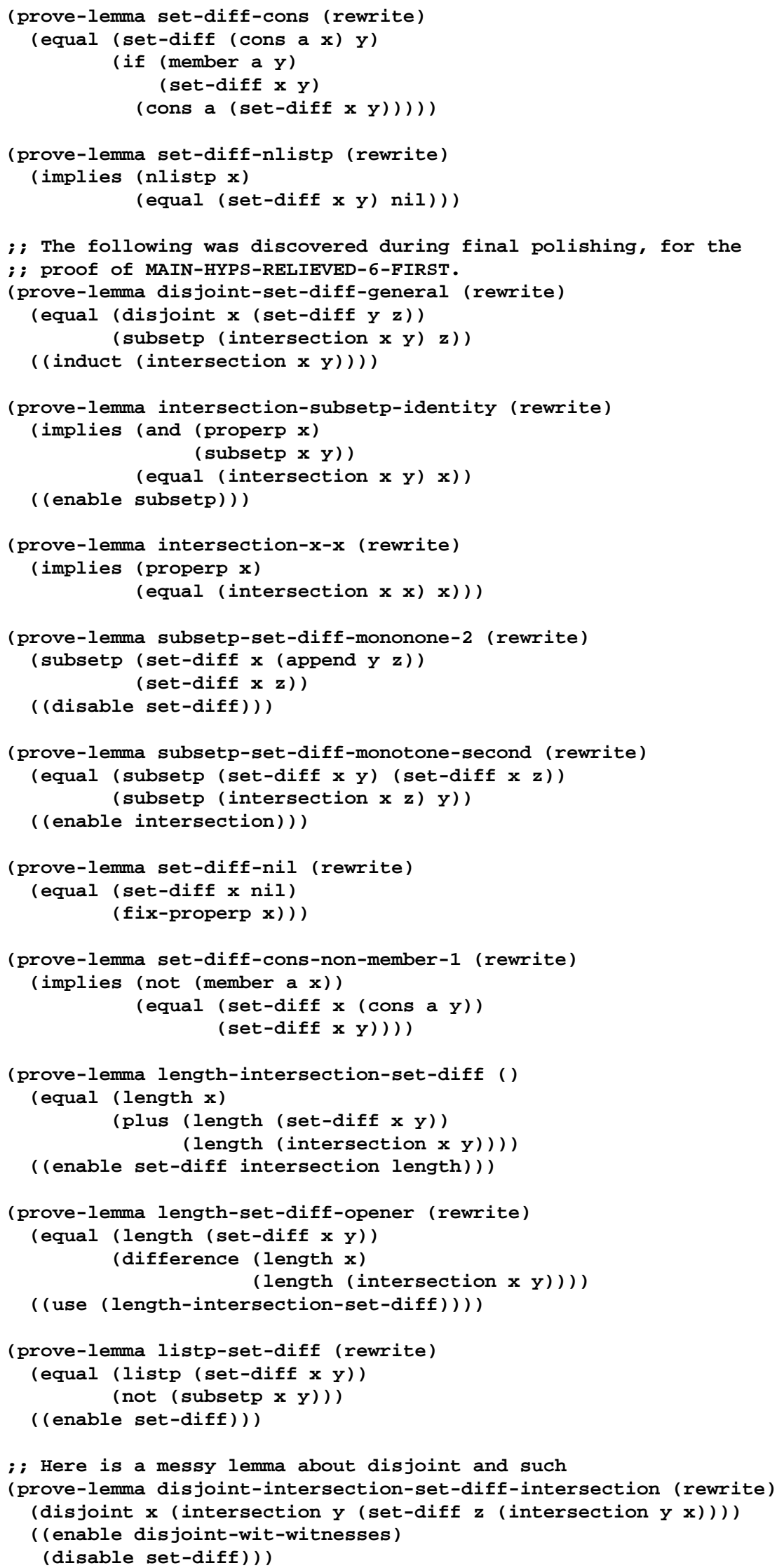




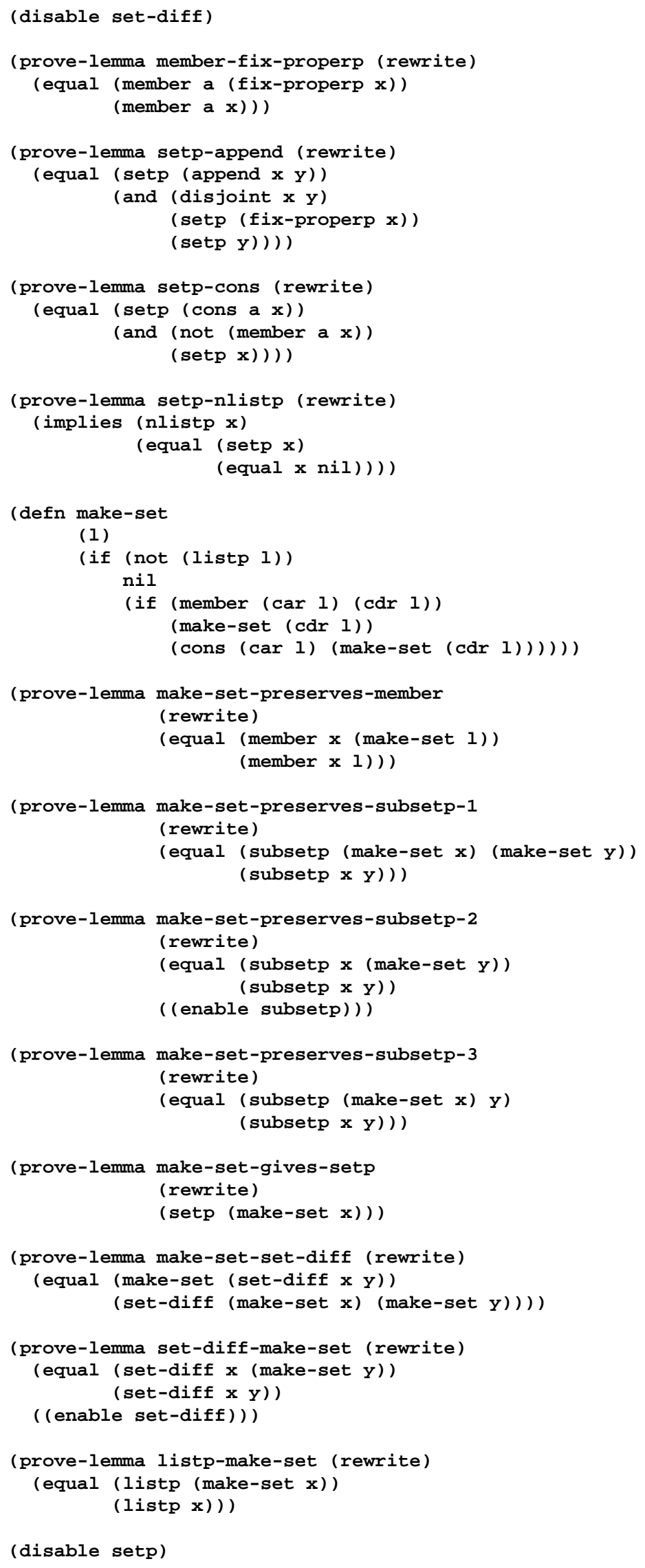




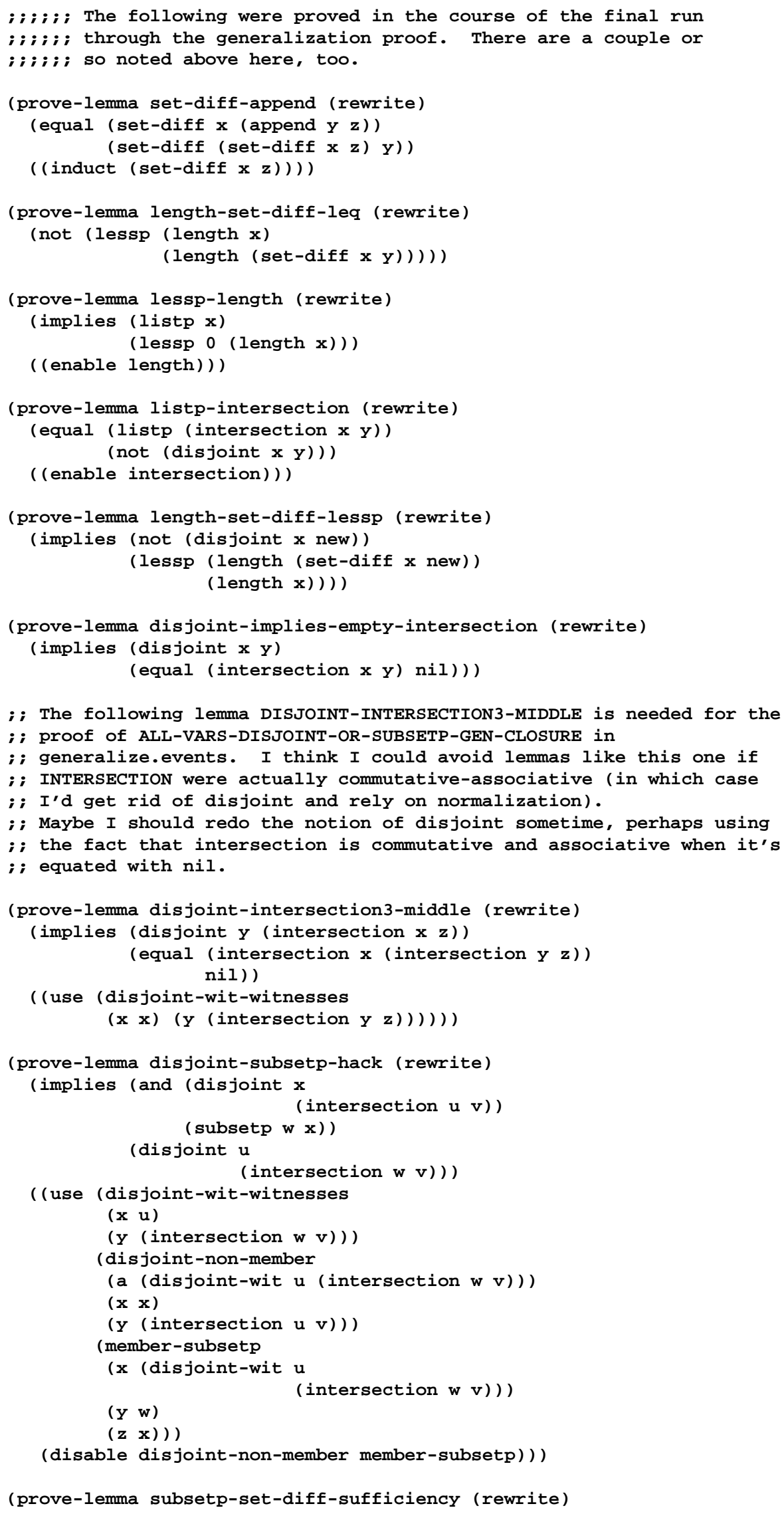




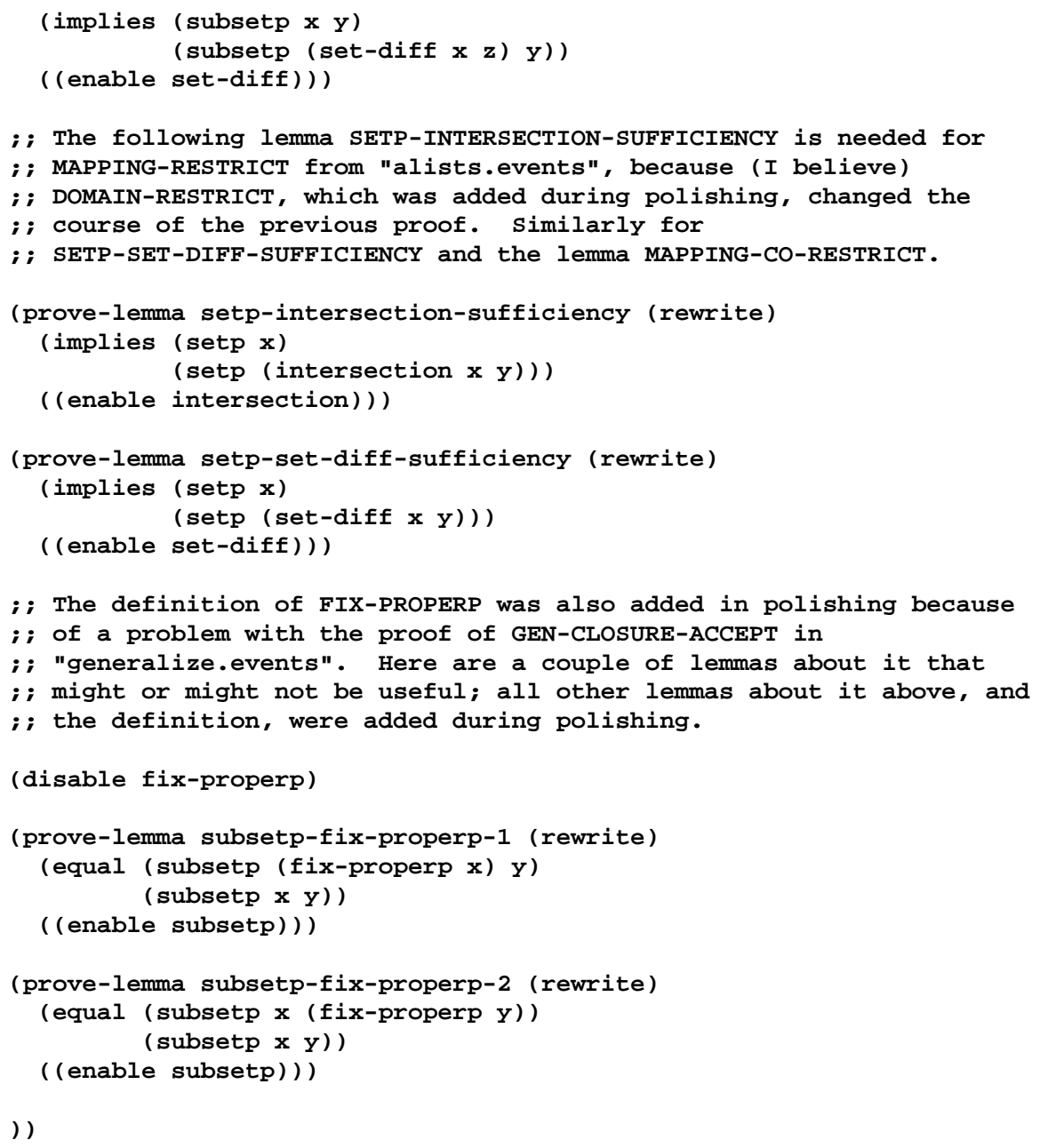


THE FILE "alists.events"

;; Requires deftheory enhancement.

; ; Requires sets.

(setq events'(

i; Alists, March 1990. Most of the definitions and some of the lemmas

;; were contributed by Bill Bevier; the rest are by Matt Kaufmann.

;; Functions defined here:

;; (deftheory alist-defns

; ; (alistp domain range value bind rembind invert mapping

i; restrict co-restrict))

(defn alistp $(x)$

(if (listp $x$ )

(and (listp (car $\mathbf{x})$ )

(alistp $(\operatorname{cdr} x))$ )

(equal $\times$ nil)))

(prove-lemma alistp-implies-properp (rewrite)

(implies (alistp $\mathbf{x}$ )

$($ properp $x))$ )

(prove-lemma alistp-nlistp (rewrite)

(implies (nlistp $x$ )

(equal (alistp $x$ )

(equal $x$ nil))))

(prove-lemma alistp-cons (rewrite)

(equal (alistp (cons a $\mathbf{x})$ )

(and (listp a)

$($ alistp $\mathbf{x})))$ )

(disable alistp)

(prove-lemma alistp-append (rewrite)

(equal (alistp (append $x y)$ )

(and (alistp (fix-properp $\mathbf{x})$ ) (alistp y))))

(defn domain (map)

(if (listp map)

(if (listp (car map))

(cons ( $\operatorname{car}(\operatorname{car} \operatorname{map}))$ (domain $(\operatorname{cdr} \operatorname{map}))$ )

(domain (cdr map)))

nil))

(prove-lemma properp-domain (rewrite)

(properp (domain map)))

(prove-lemma domain-append (rewrite)

(equal (domain (append $x y)$ )

(append (domain $\mathbf{x})$ (domain $\mathbf{y}))$ ))

(prove-lemma domain-nlistp (rewrite)

(implies (nlistp map)

(equal (domain map) nil)))

(prove-lemma domain-cons (rewrite)

(equal (domain (cons a map))

(if (listp a)

(cons (car a) (domain map))

(domain map))))

(prove-lemma member-domain-sufficiency (rewrite)

(implies (member (cons a x) $y$ ) 


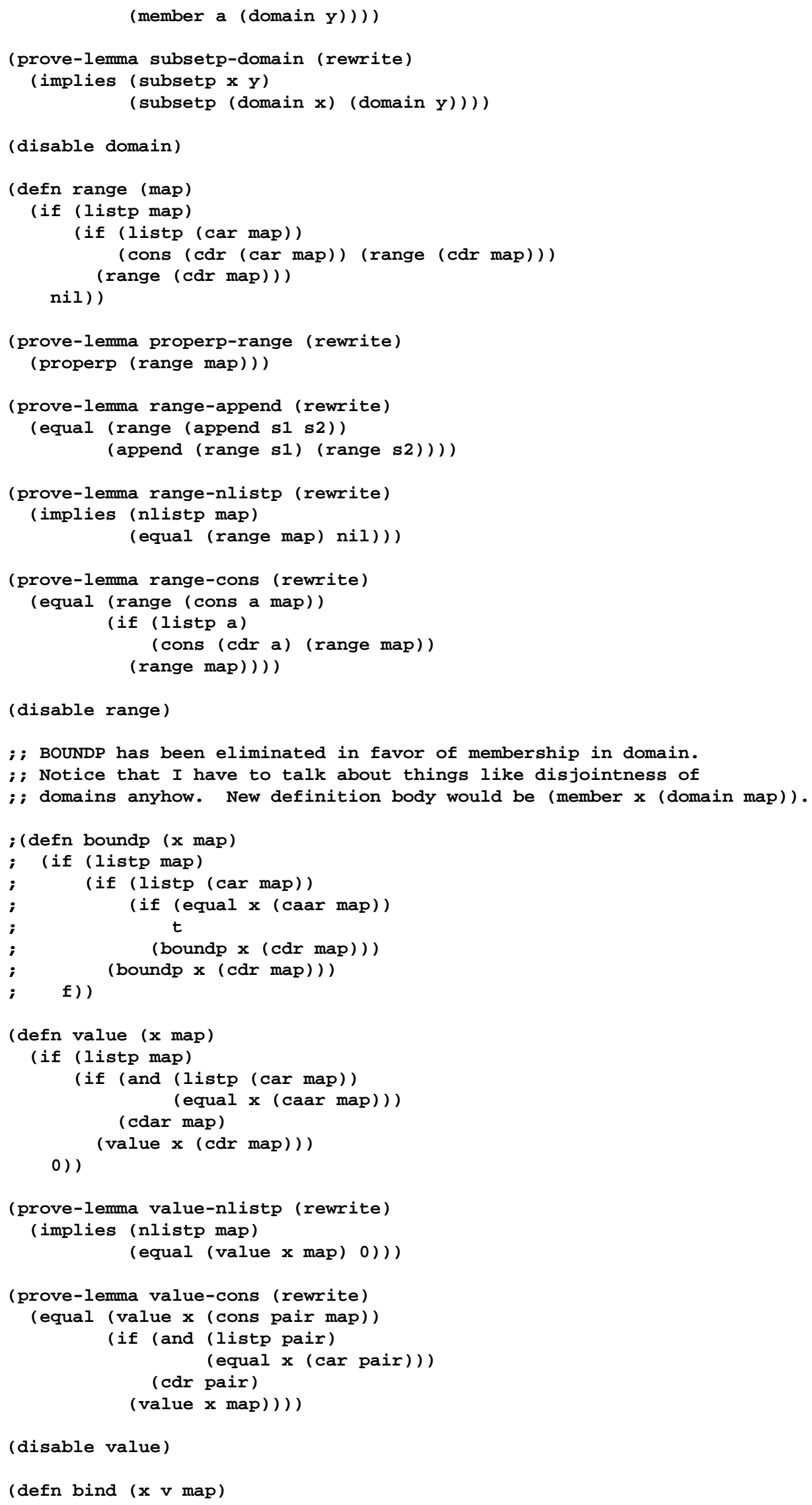




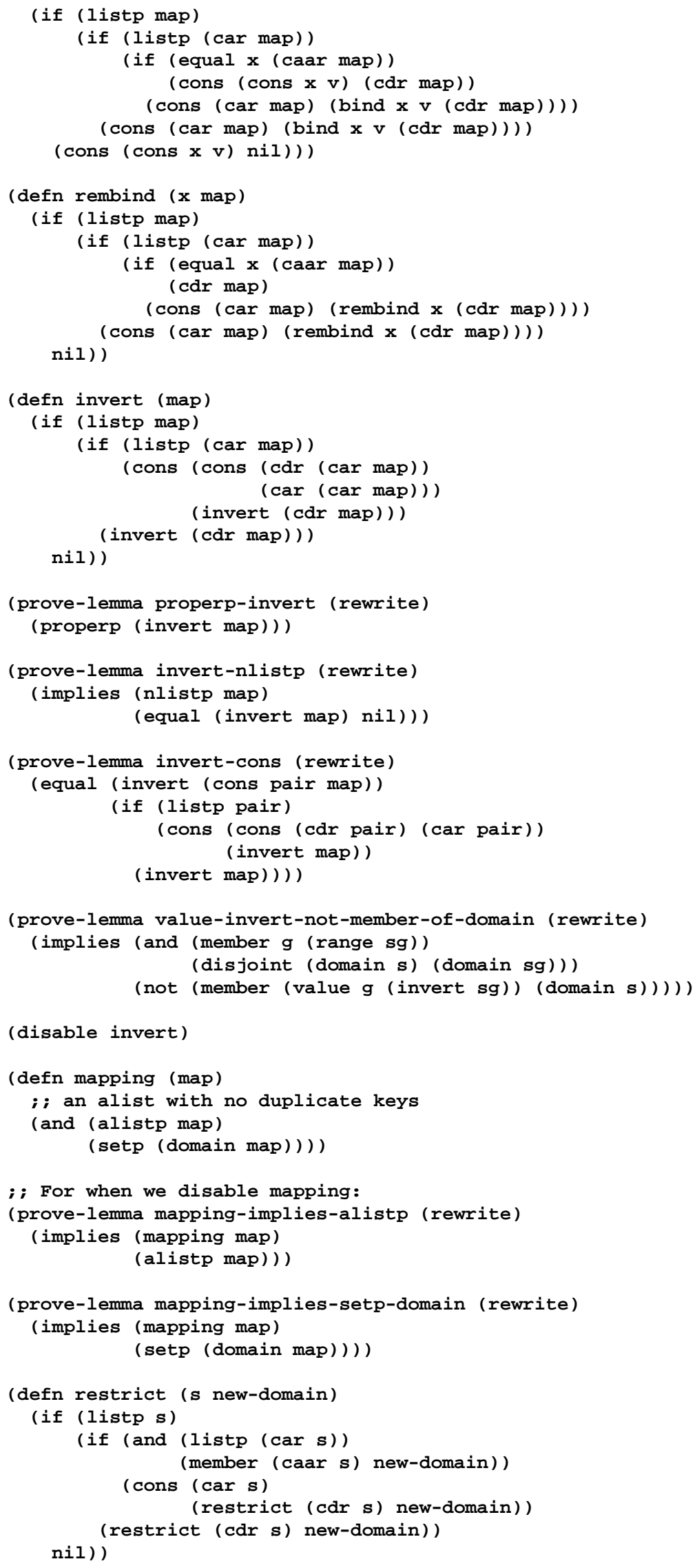




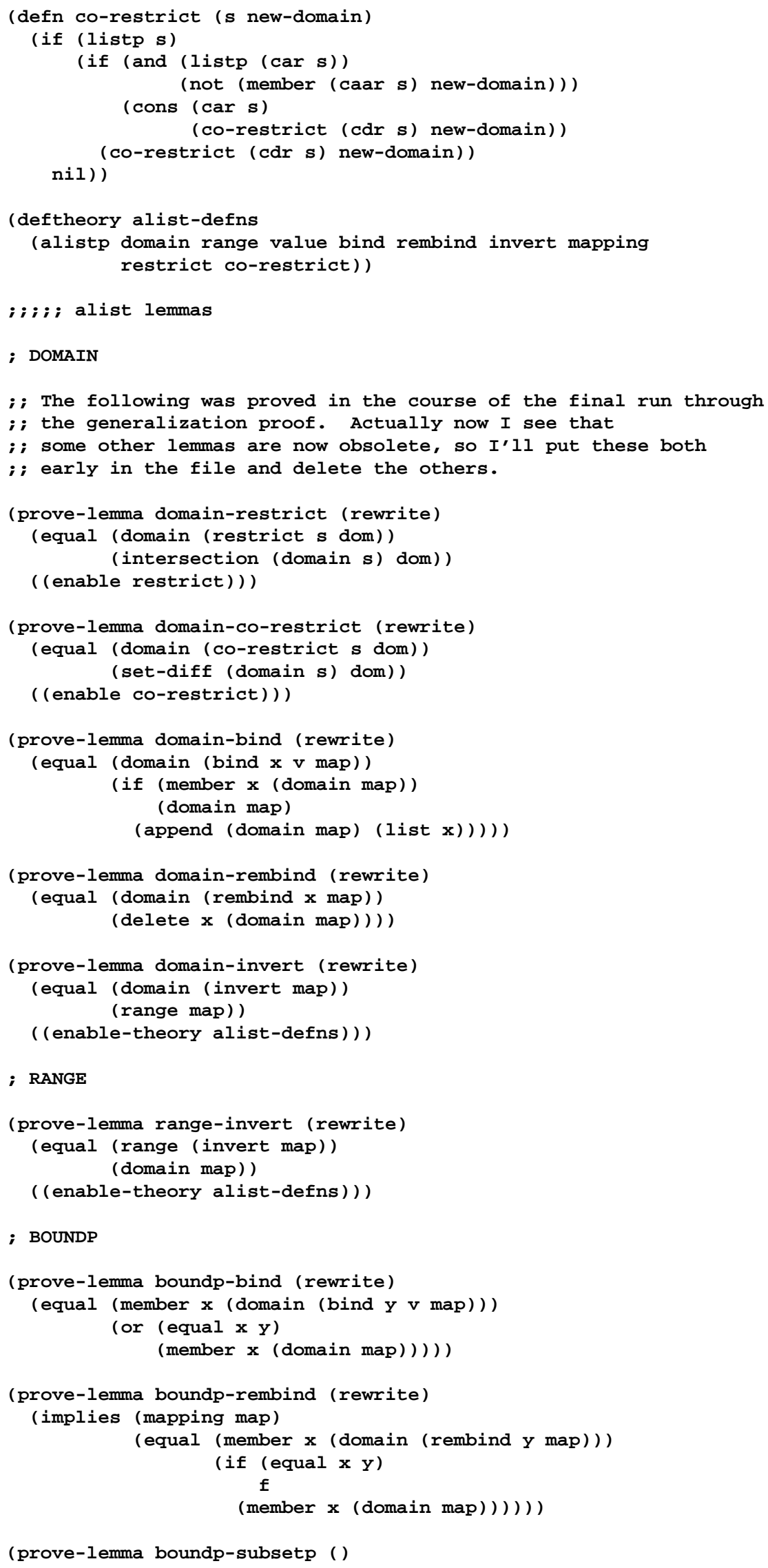




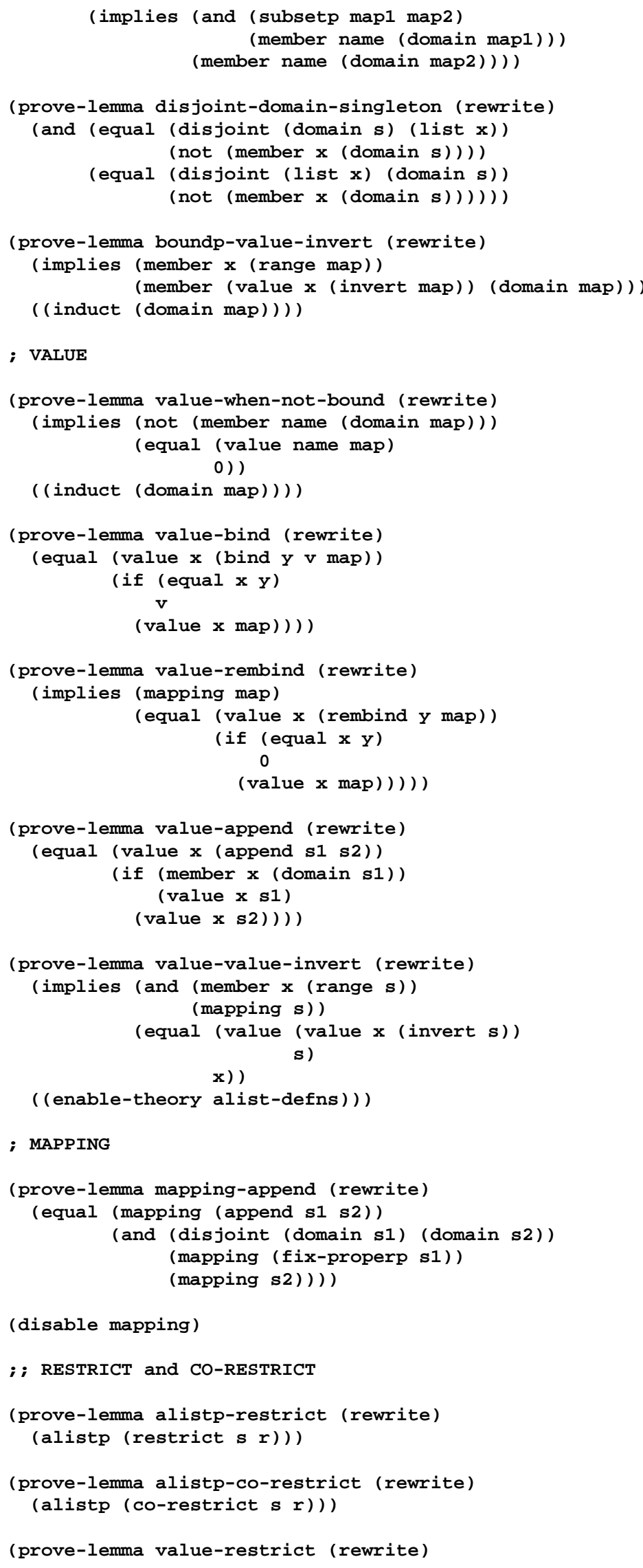




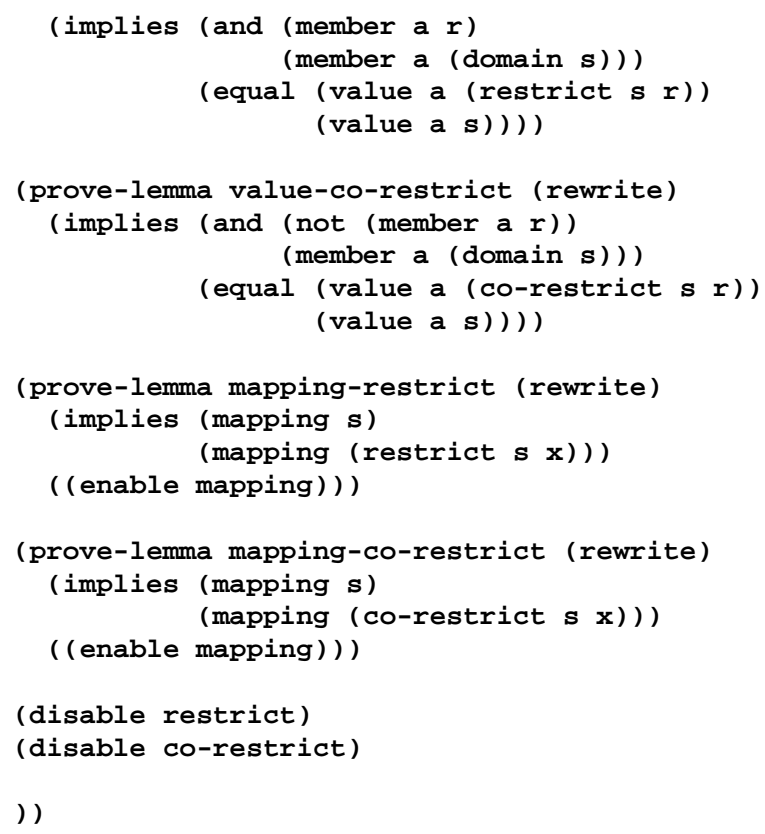




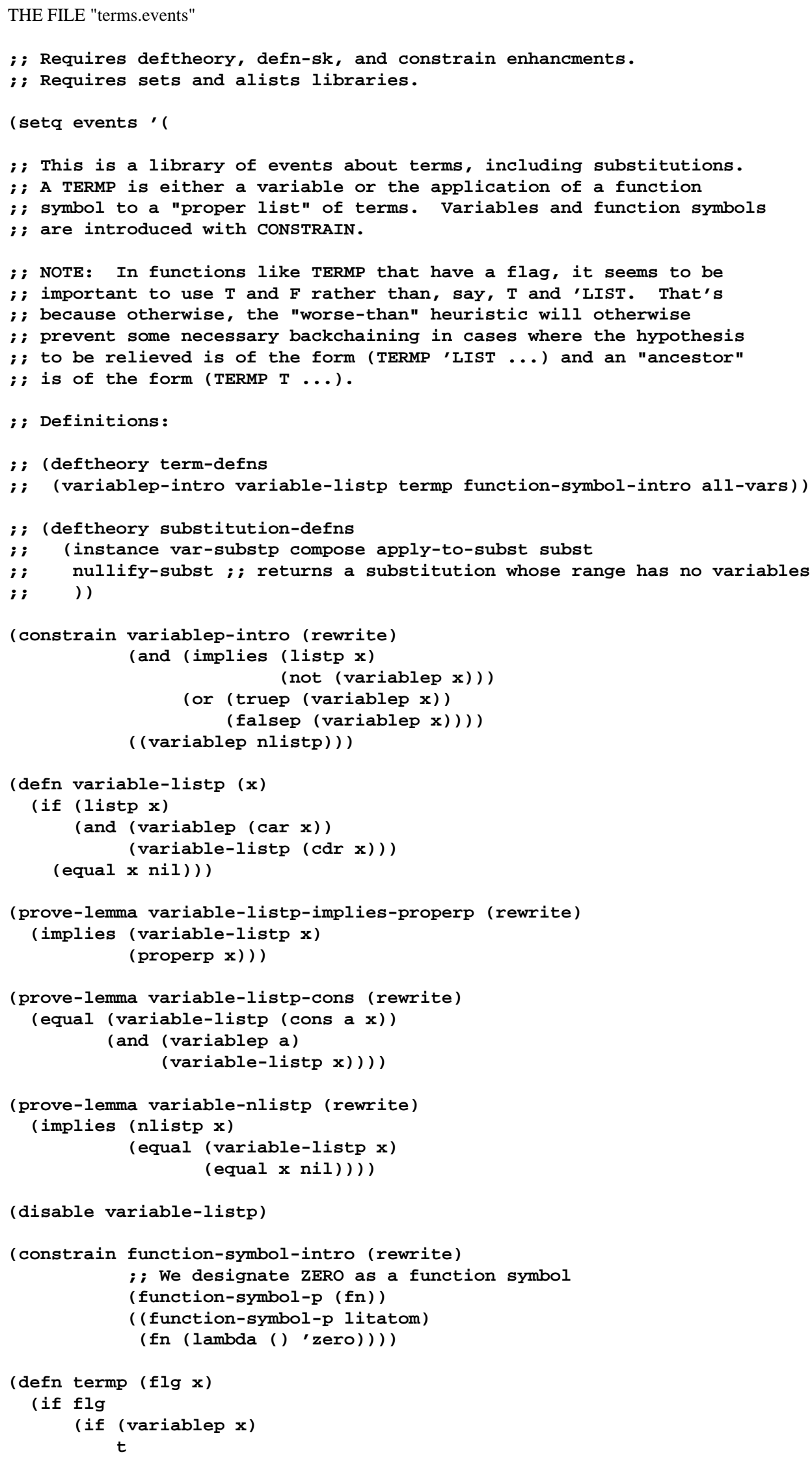




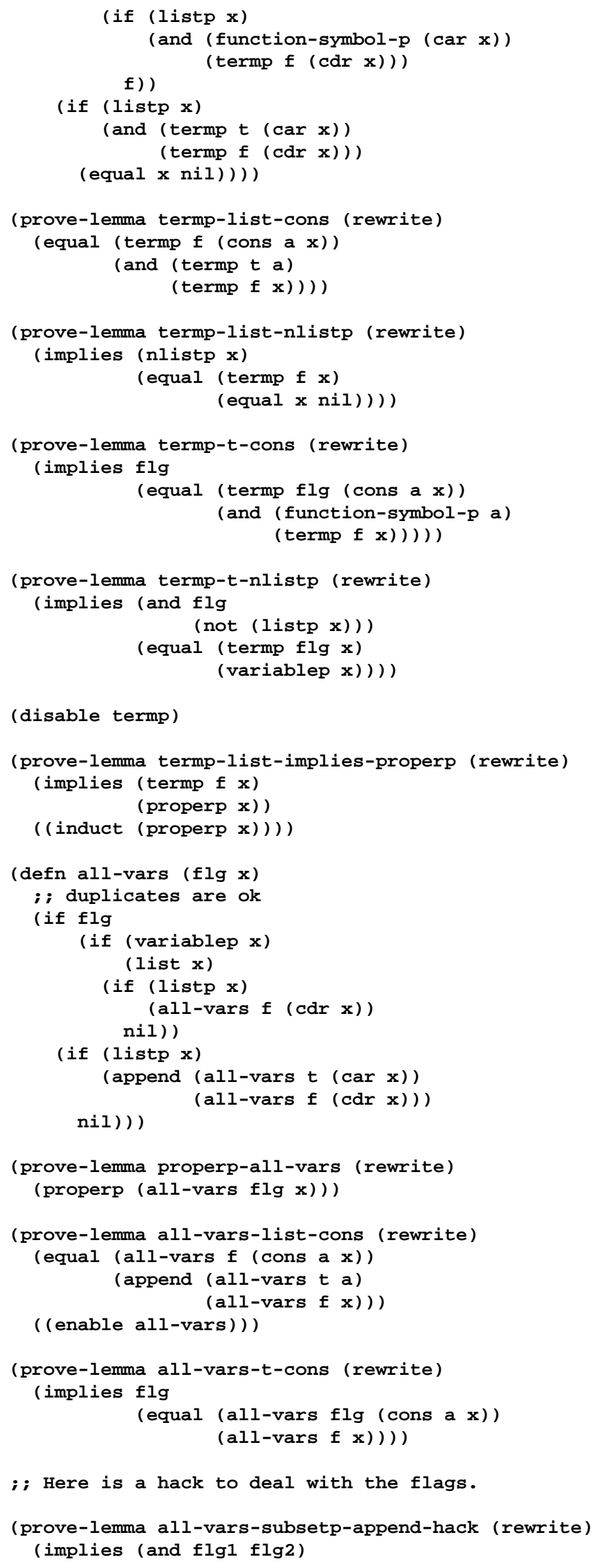




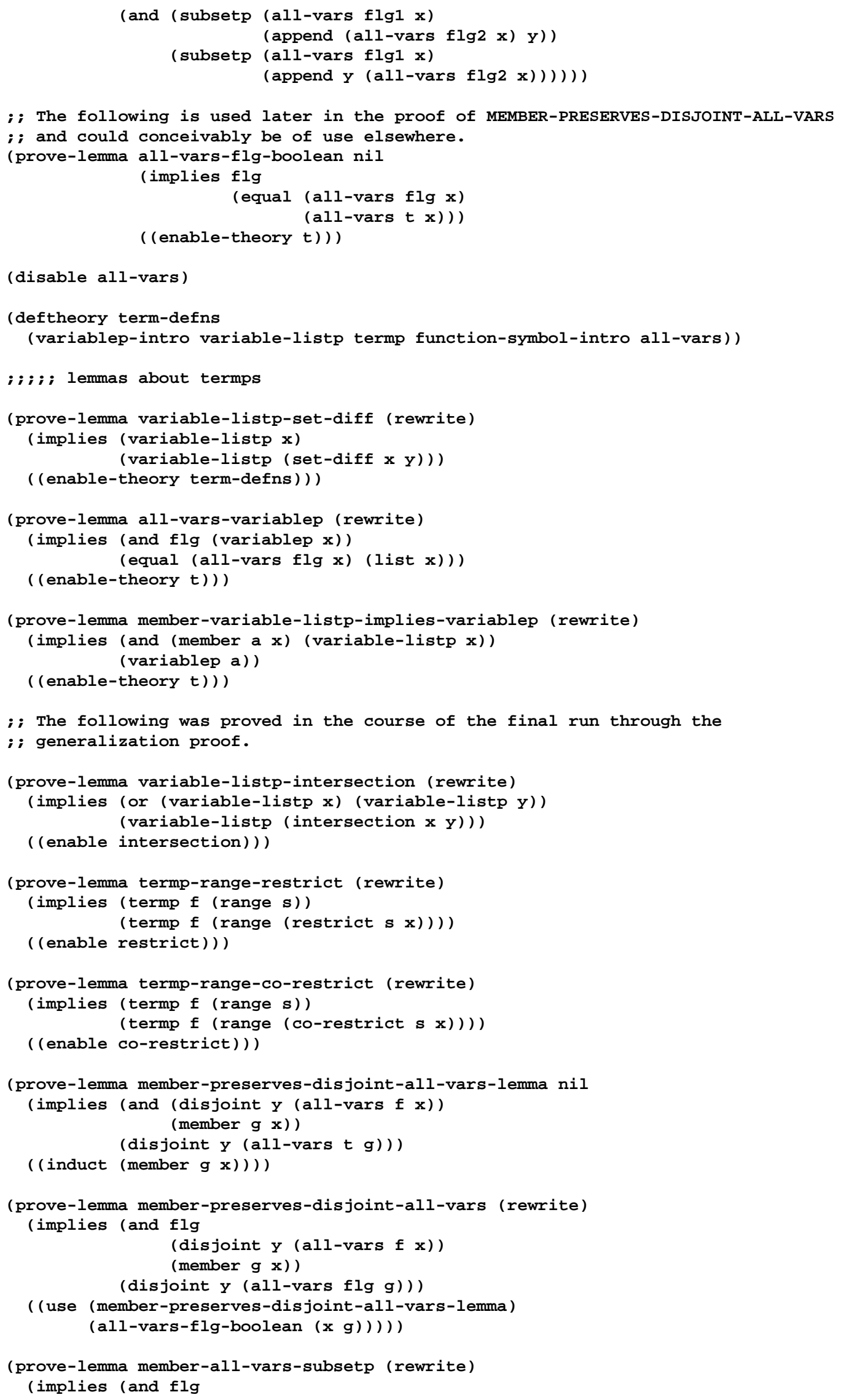




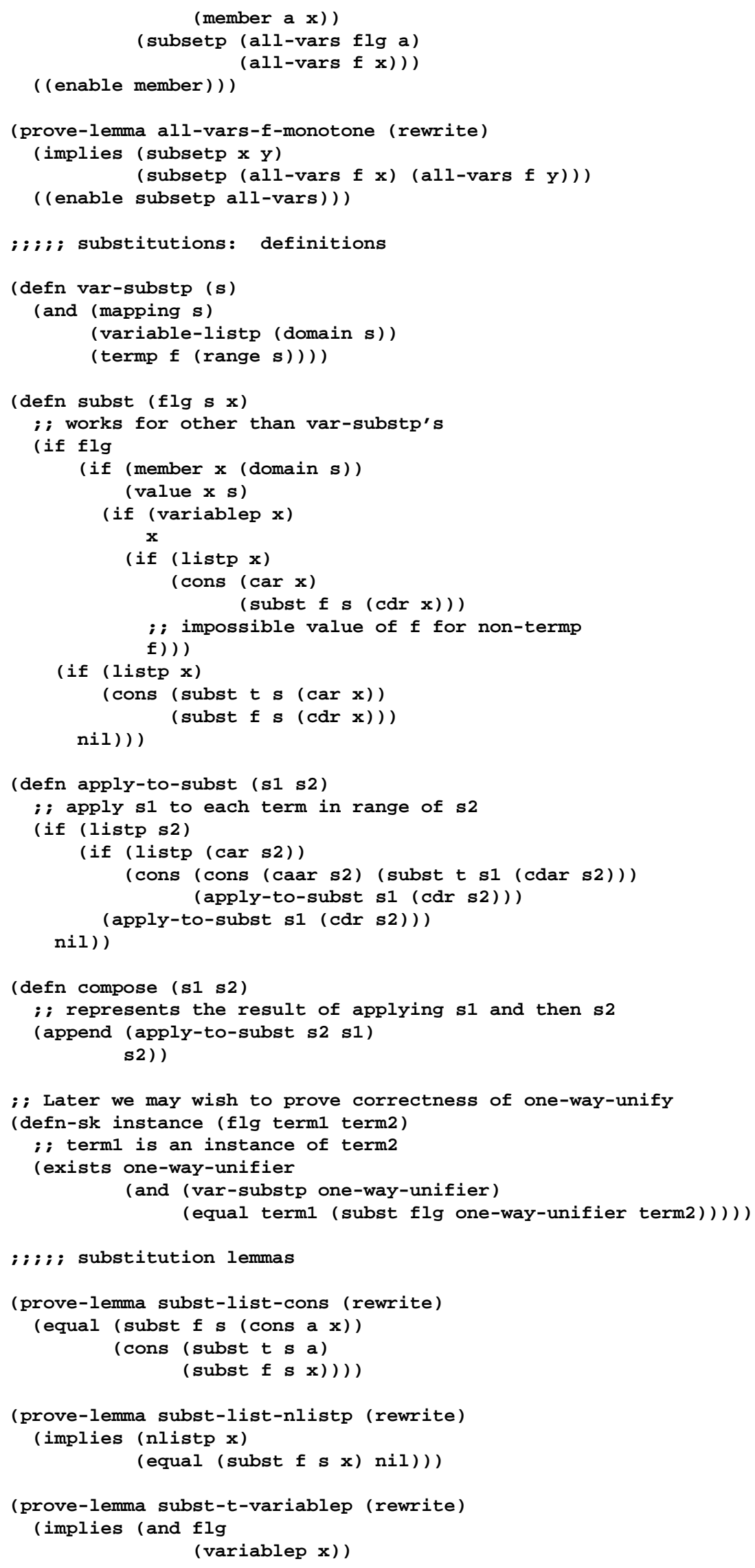




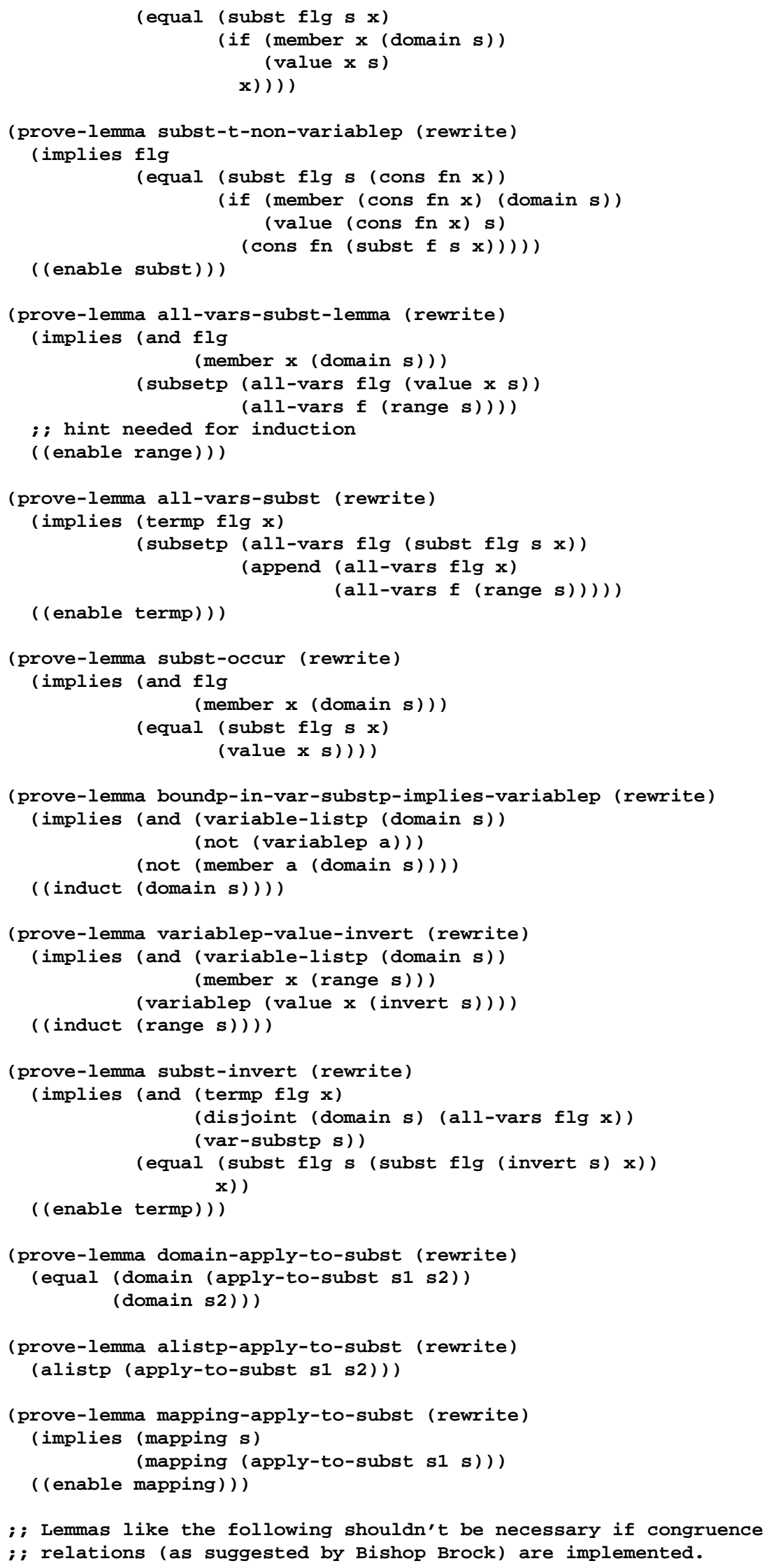




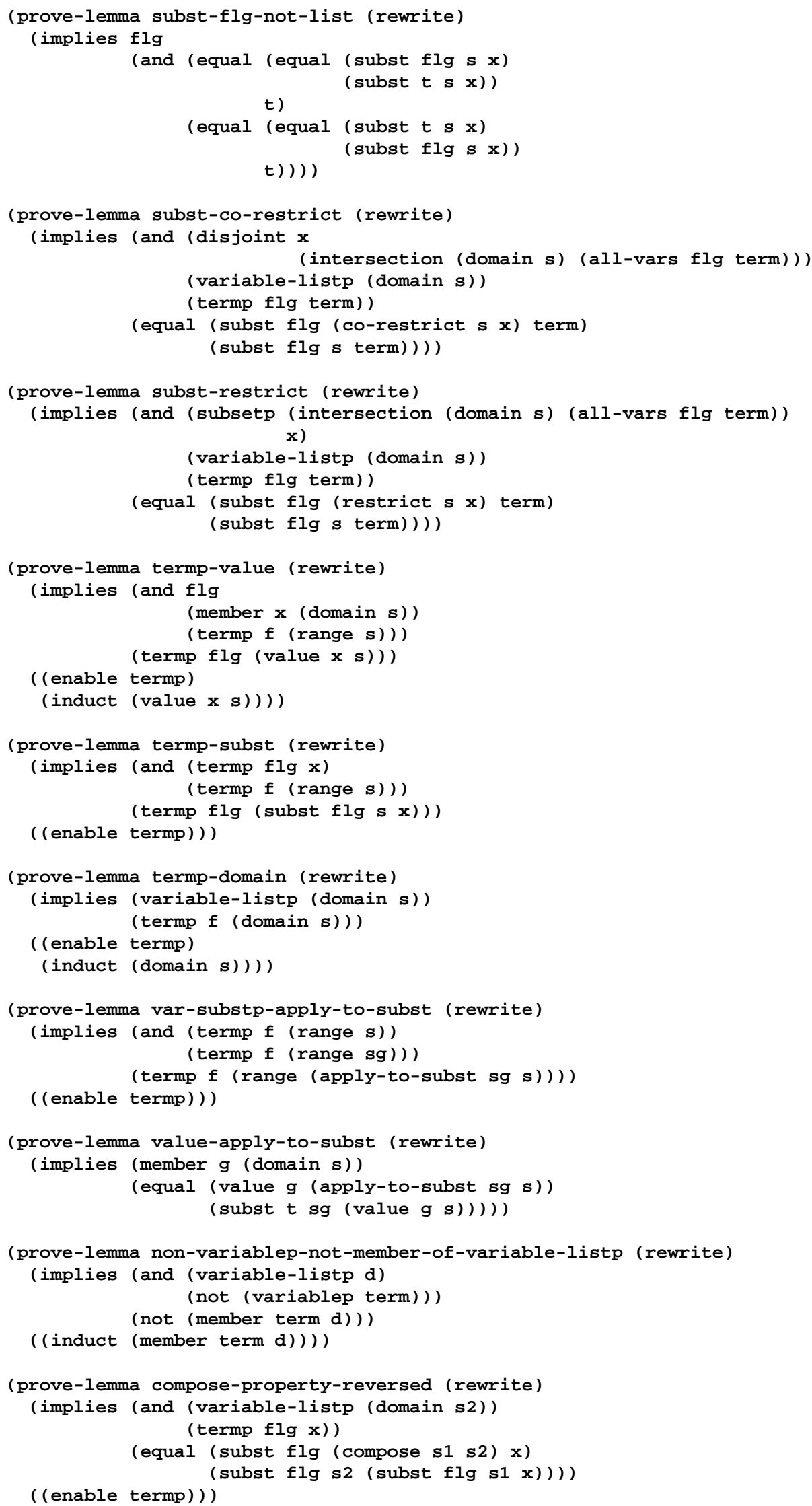




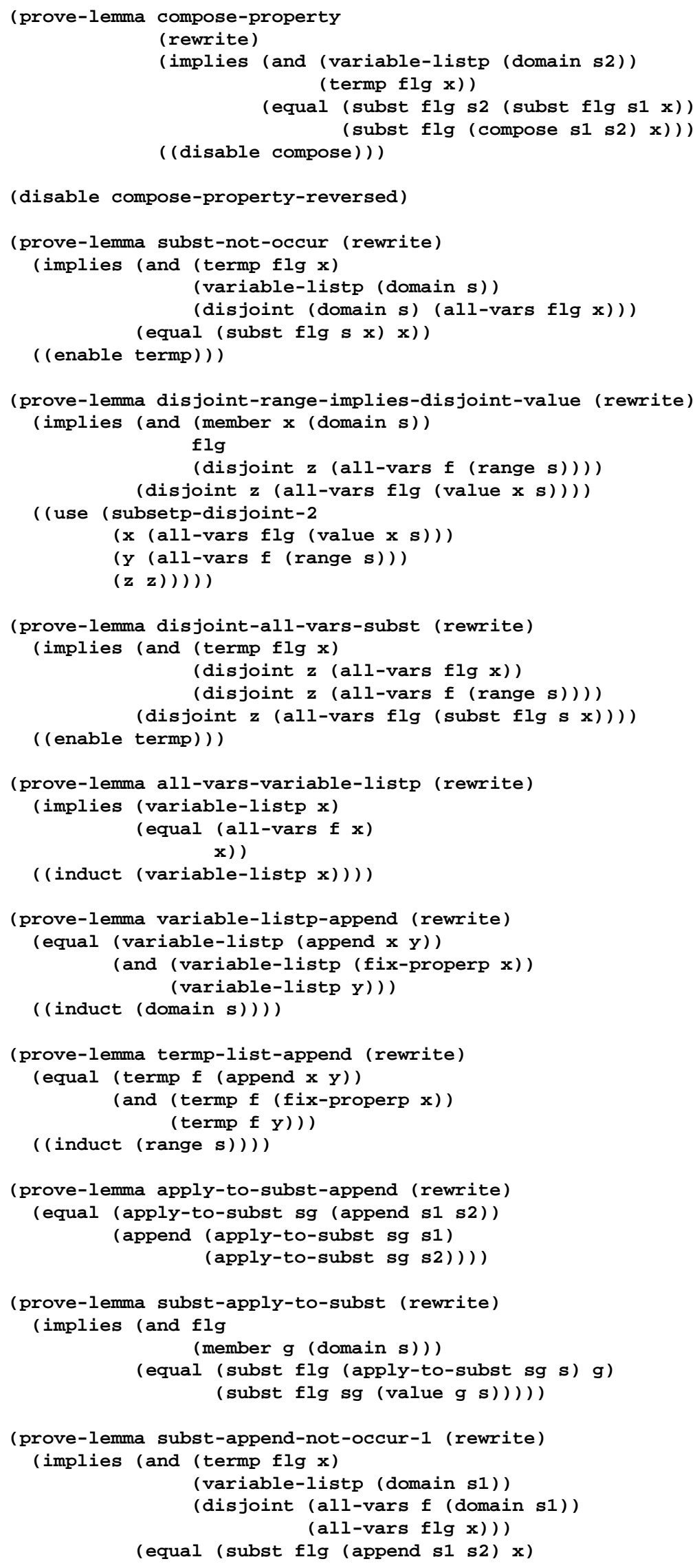




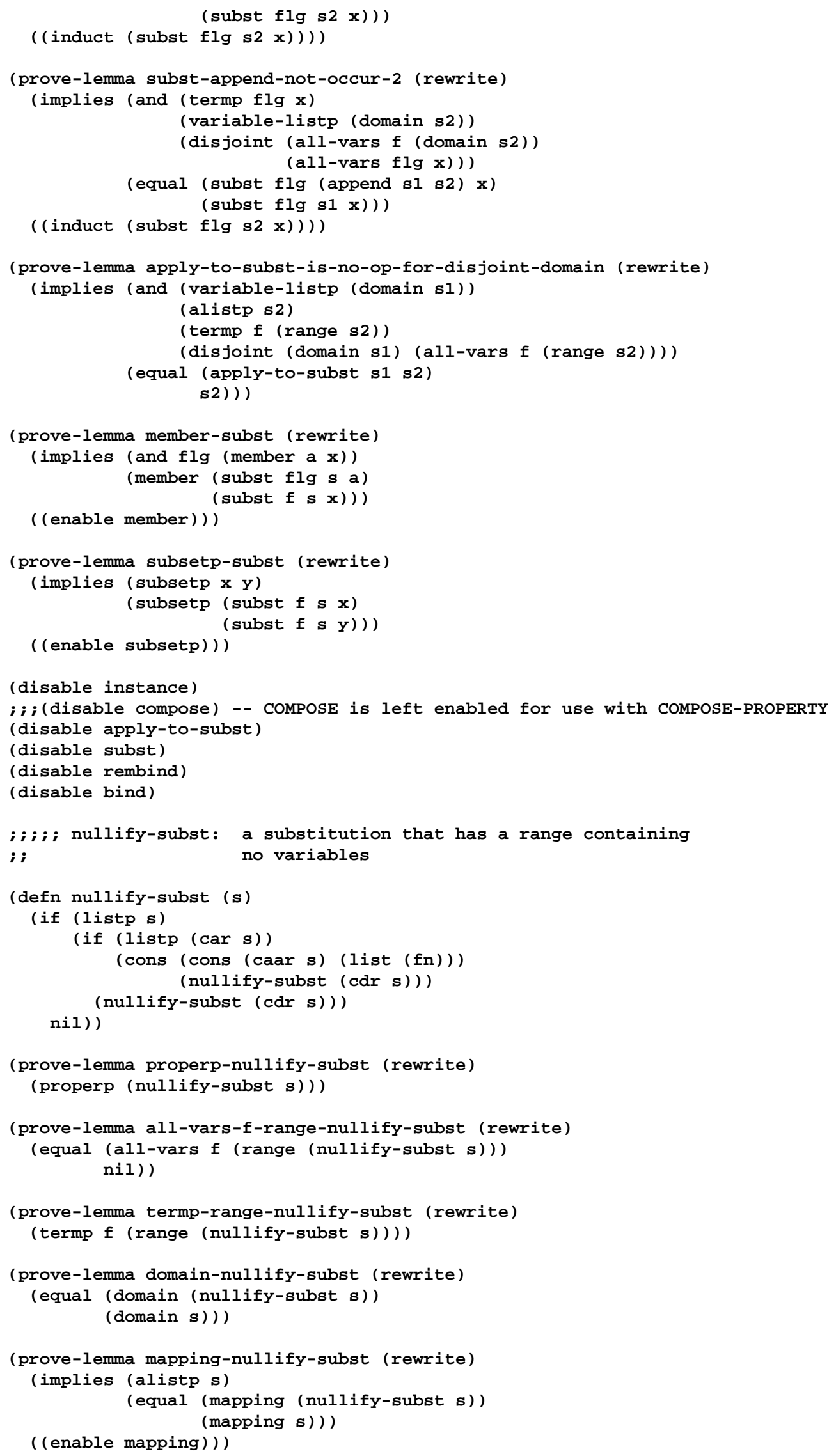




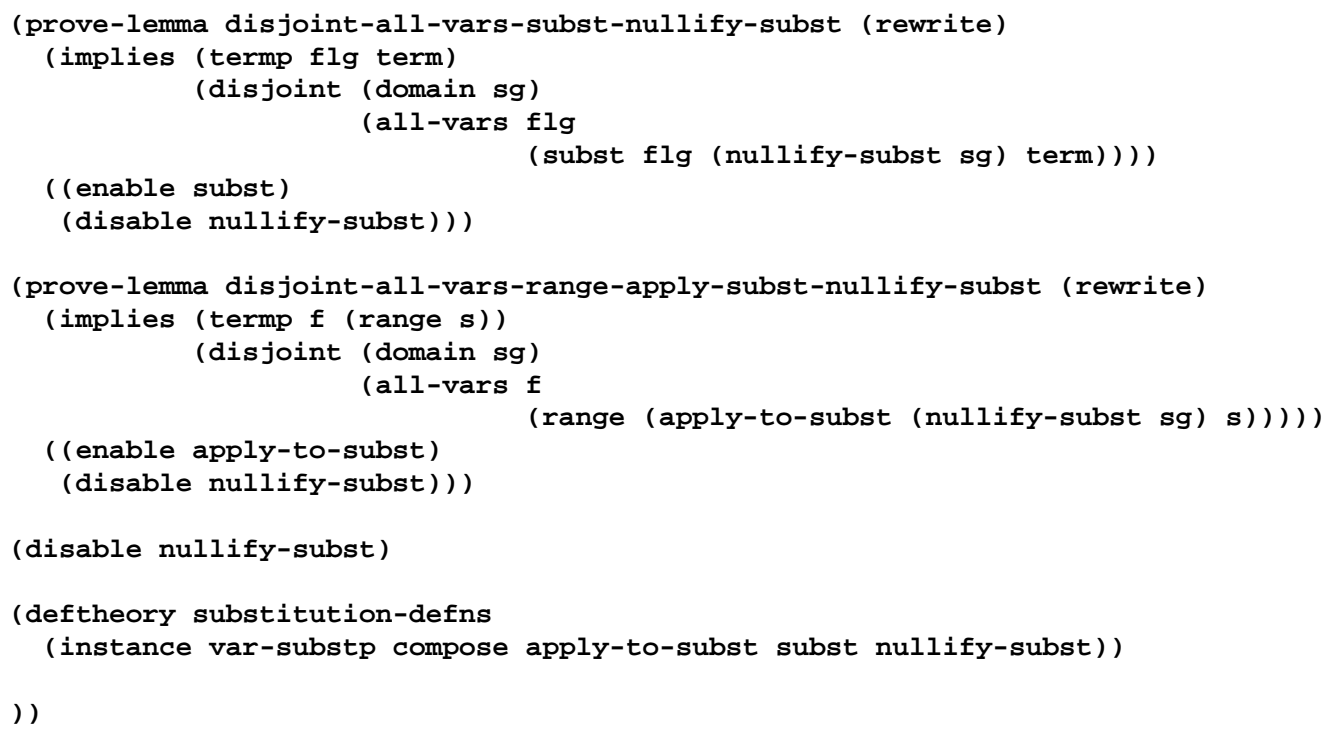


THE FILE "generalize.events"

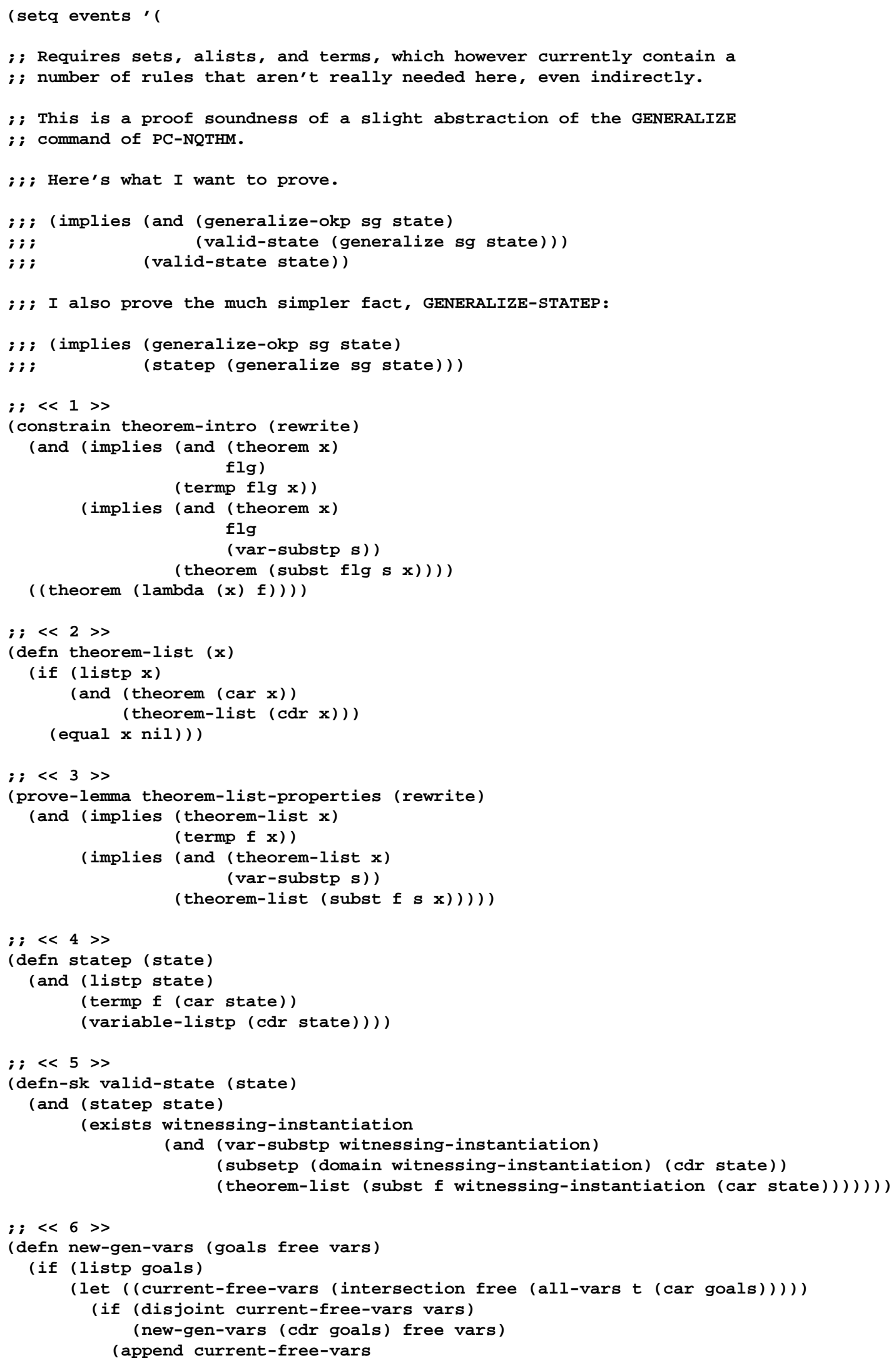




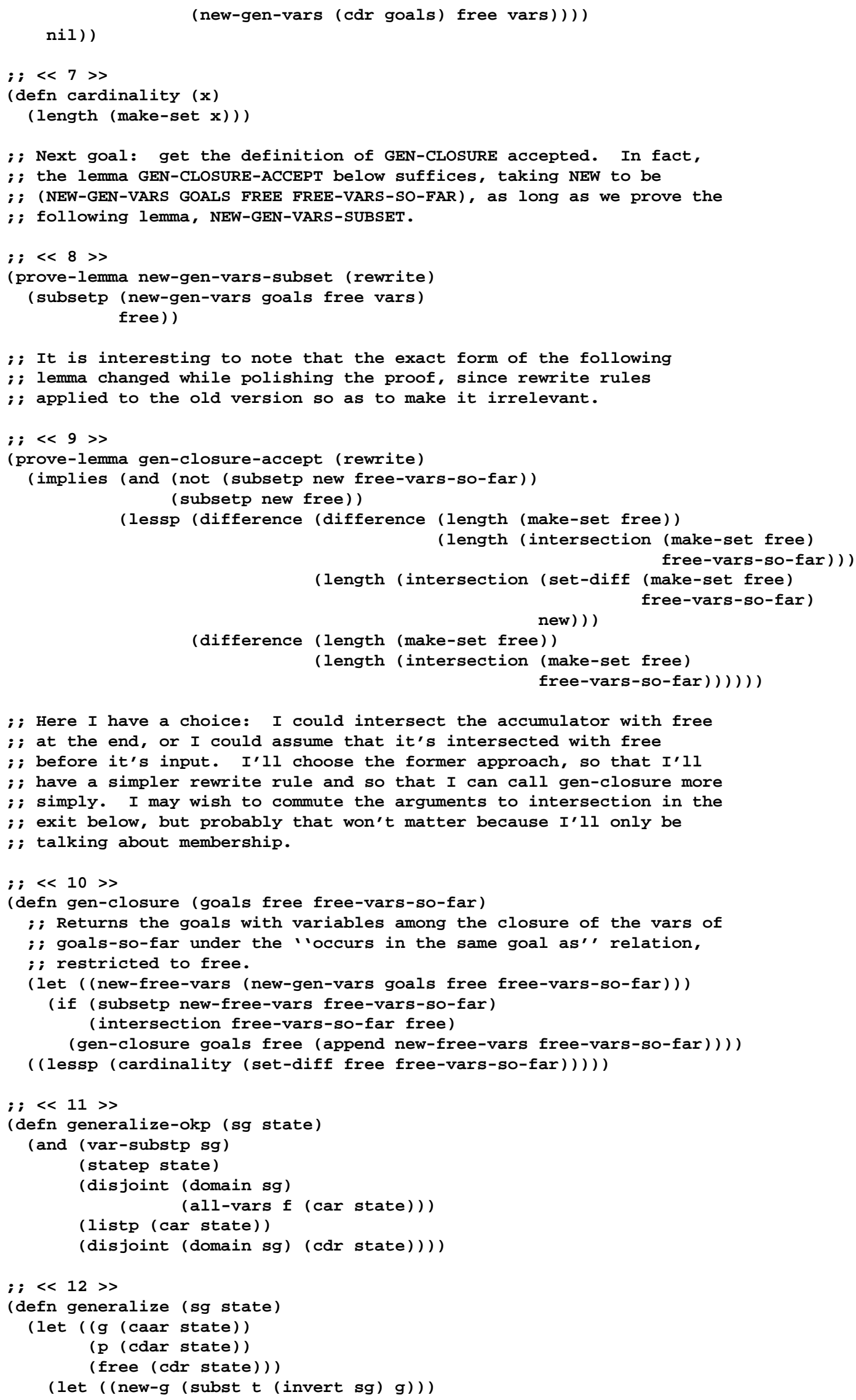




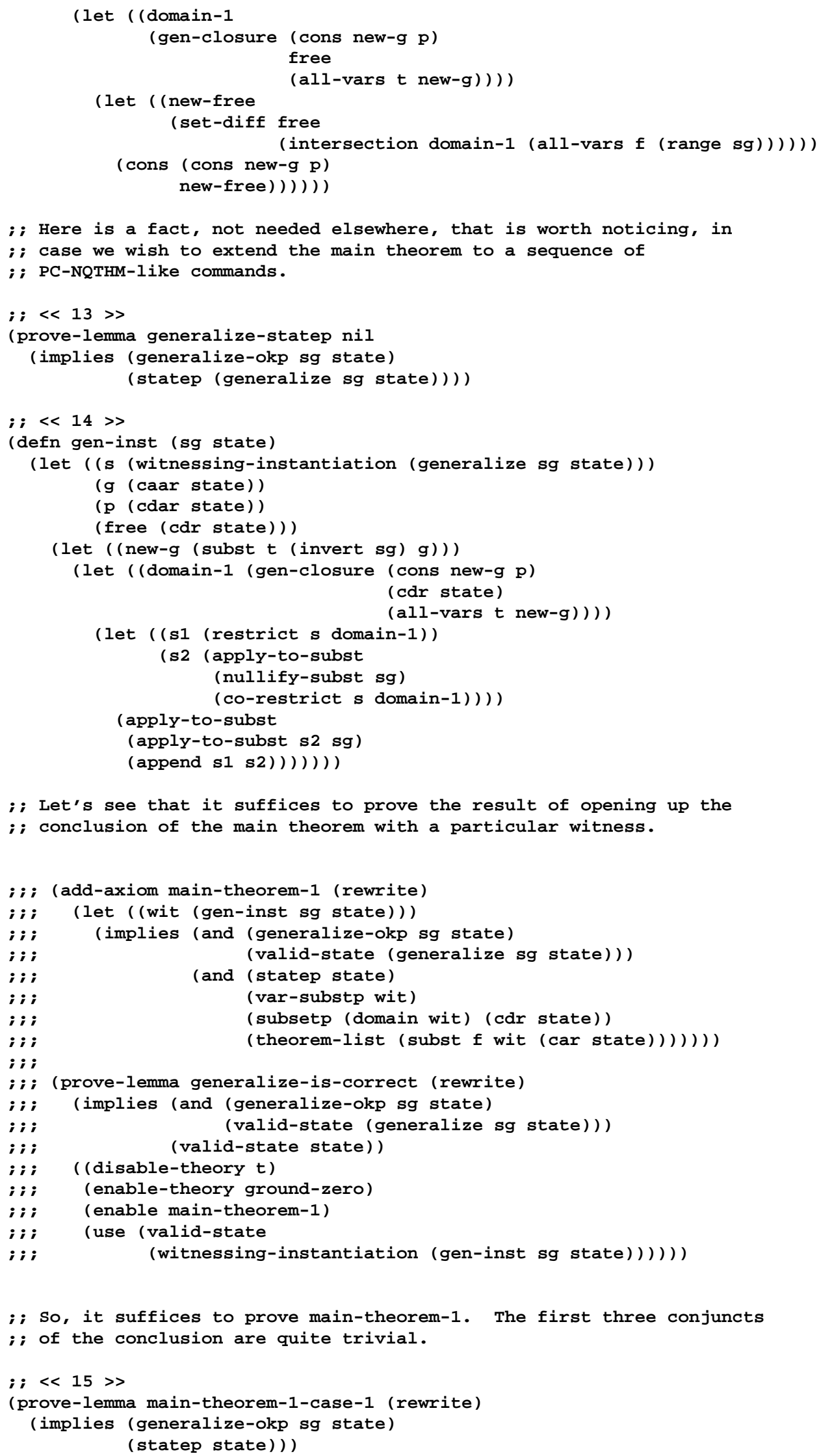




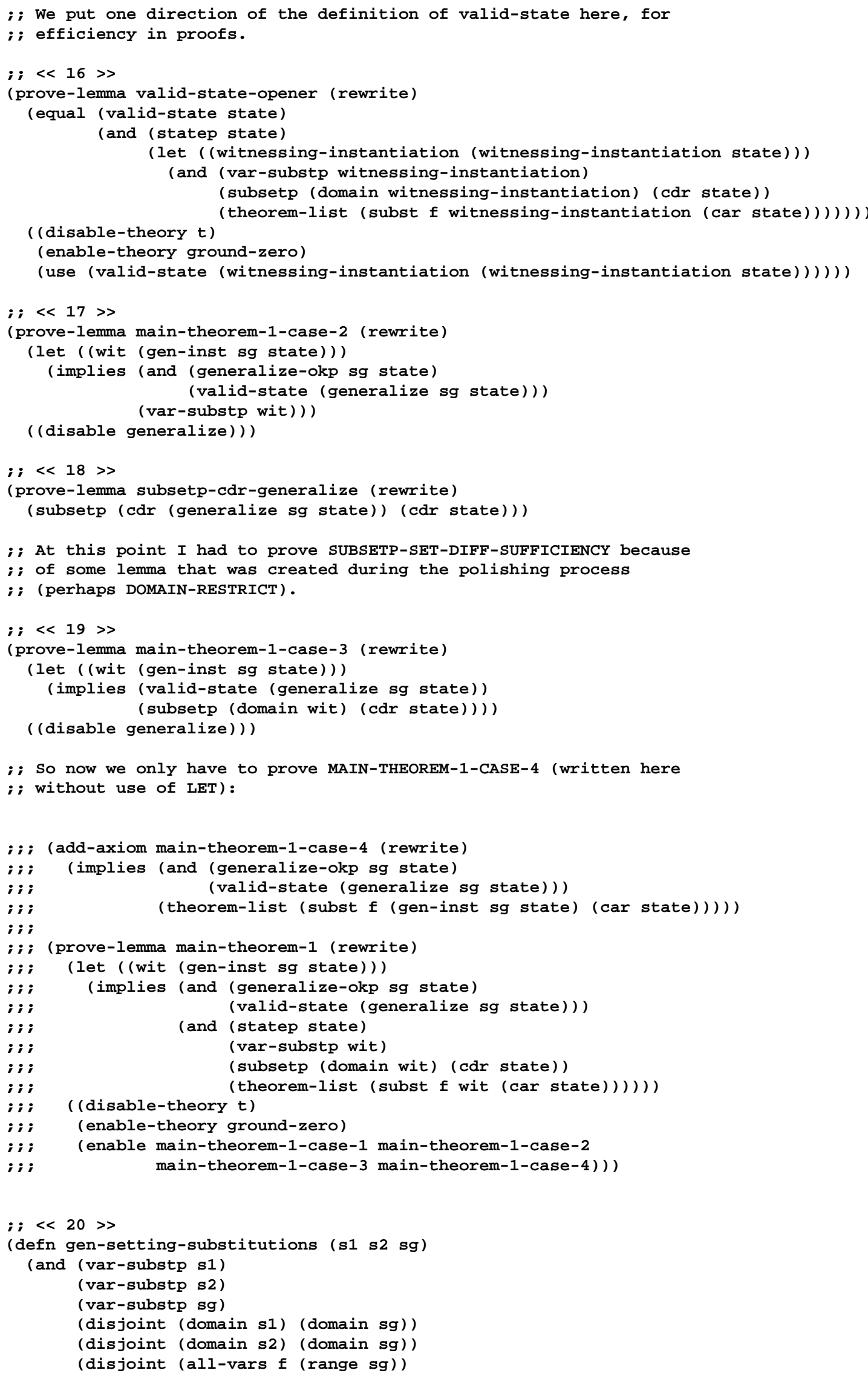




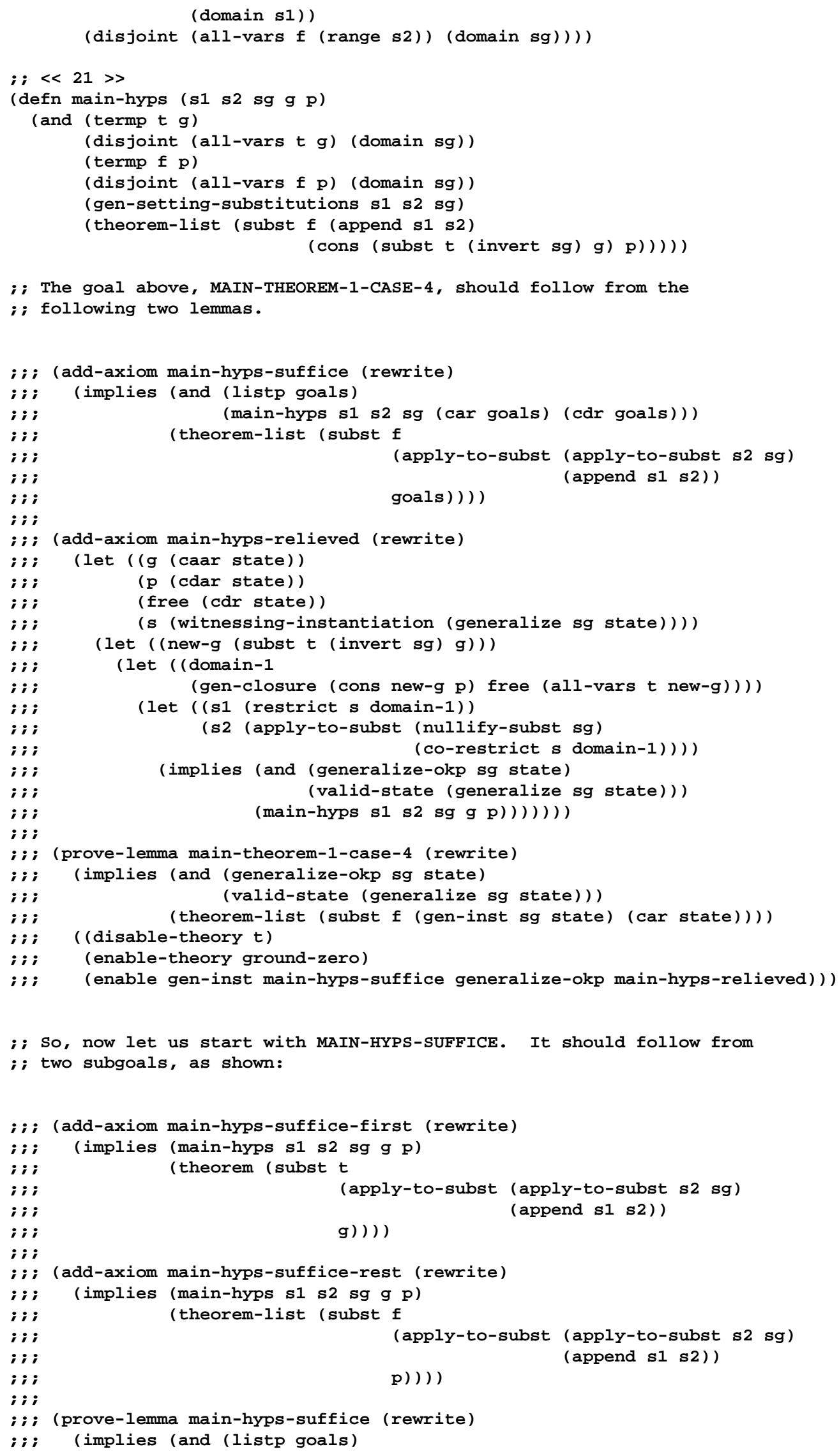




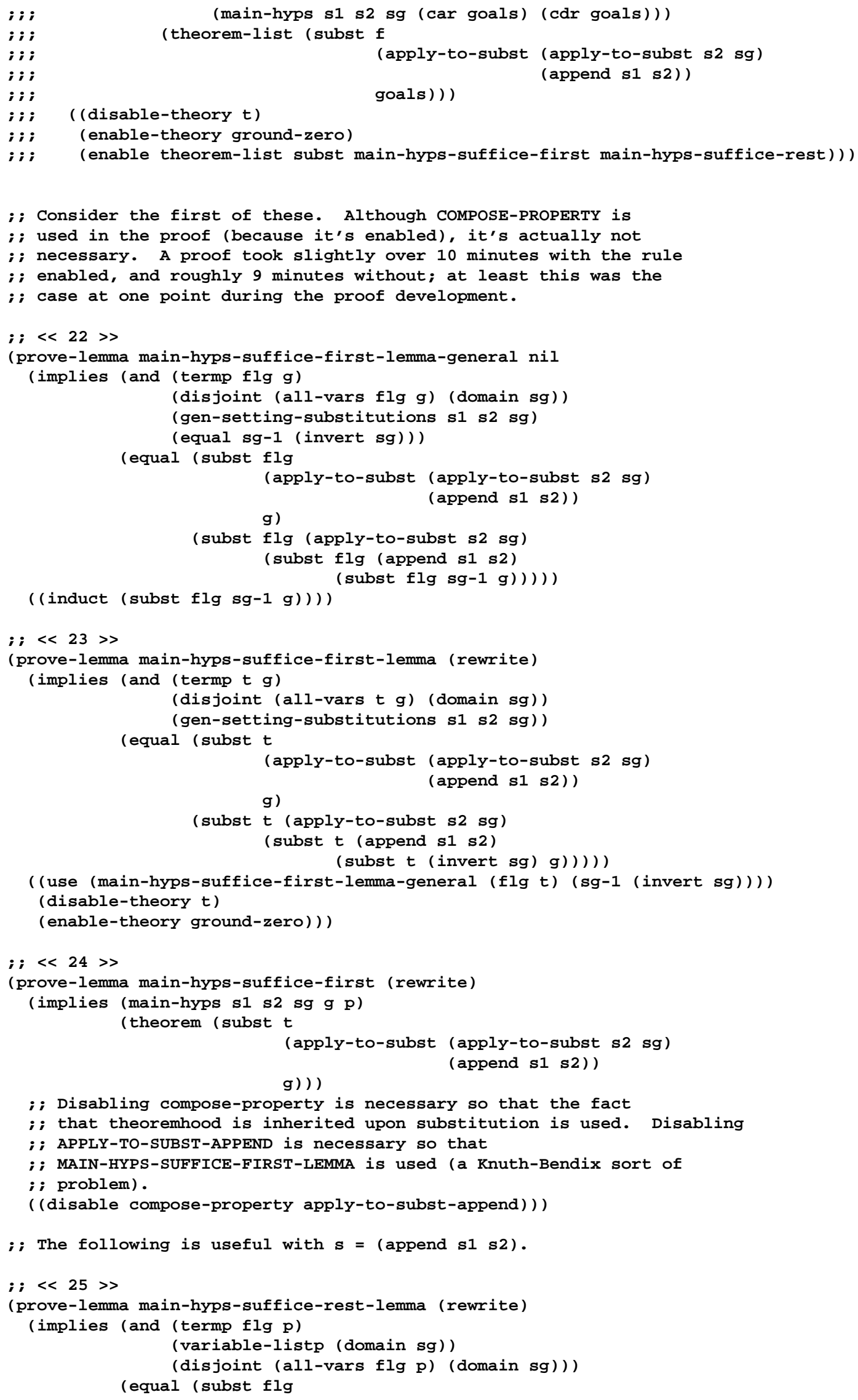




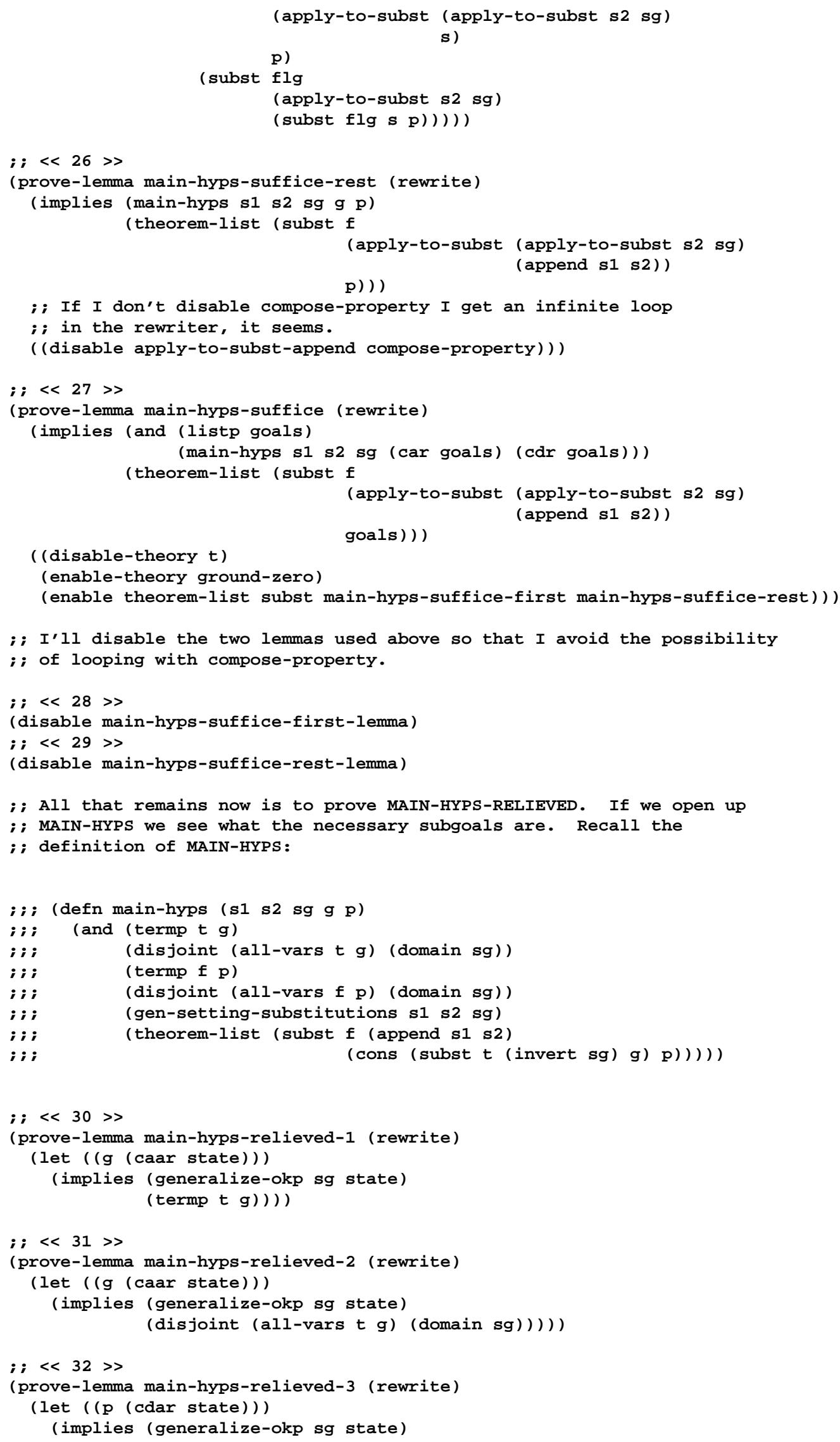




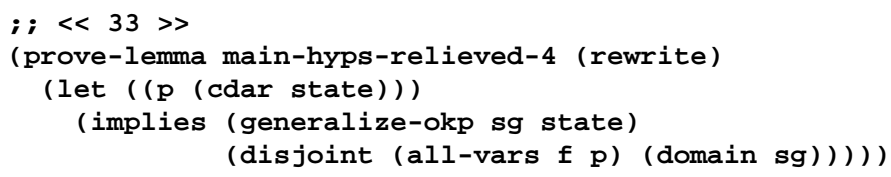


;; (probably not necessary, though I didn't let the prover run long

; ; enough to find out for sure).

; ; $<34 \gg$

(prove-lemma main-hyps-relieved-5-lemma-1 (rewrite)

(let ((s (witnessing-instantiation (generalize sg state))))

(let ( (s1 (restrict $s$ domain-1))

(s2 (apply-to-subst (nullify-subst sg)

(co-restrict s domain-1)) ))

(implies (valid-state (generalize sg state))

(and (var-substp $s 1$ )

$($ var-substp s2)))))

((disable generalize)) )

;; The next case is trivial.

; ; 35 < $>$

(prove-lemma main-hyps-relieved-5-lemma-2 (rewrite)

(implies (generalize-okp sg state)

$($ var-substp sg)))

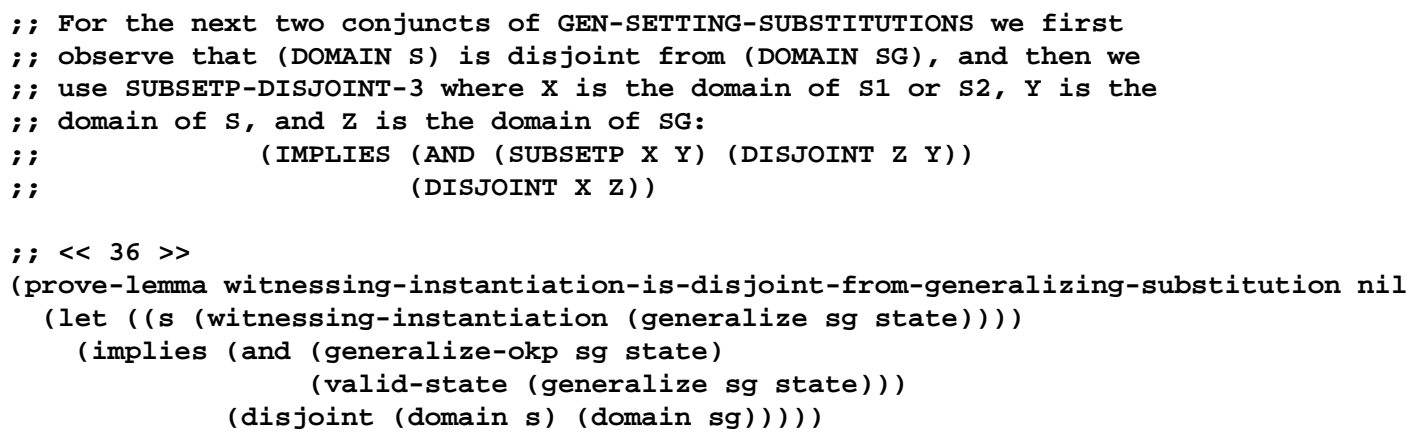




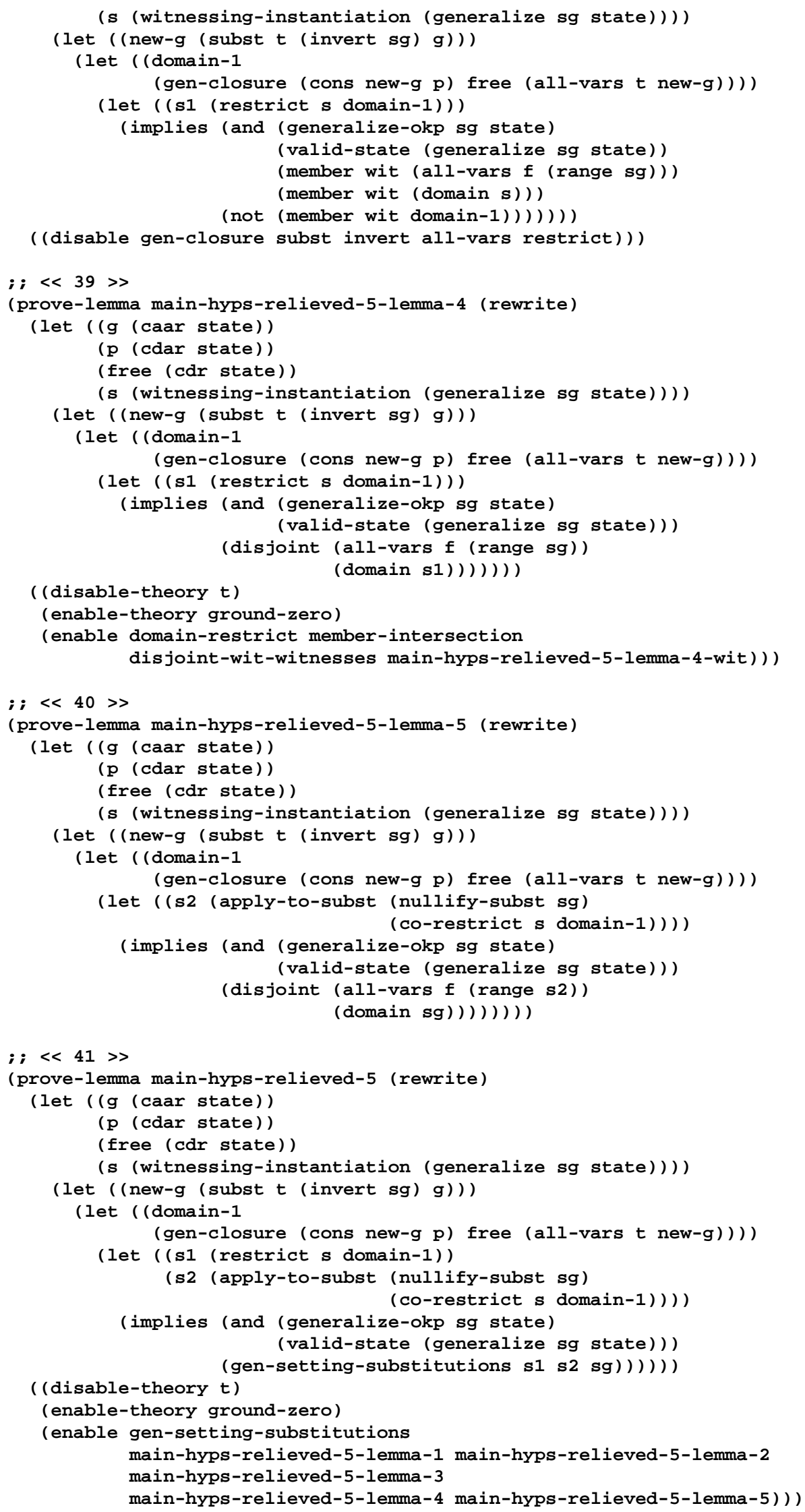




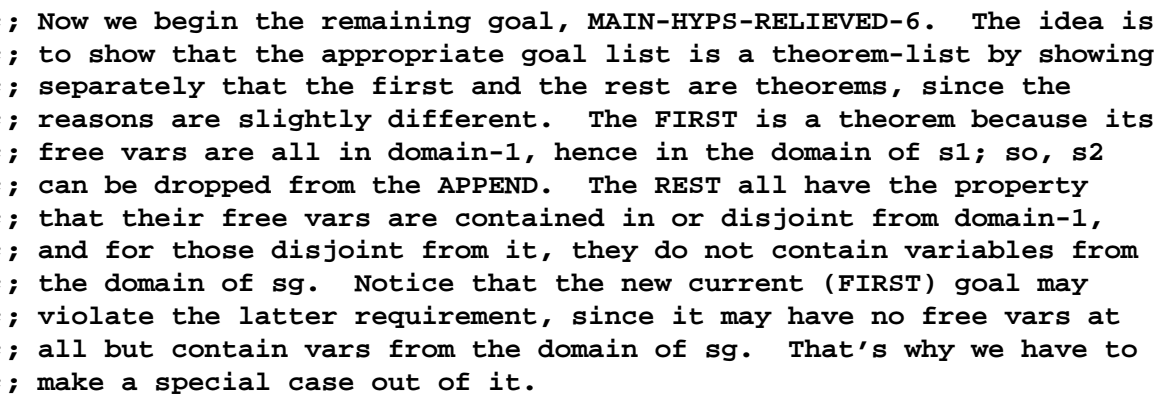


; ; adding abbreviations and promoting hypotheses) and saw that I

; ; wanted to rewrite with SUBST-APPEND-NOT-OCCUR-2. I also notice the

; ; need for GEN-CLOSURE-CONTAINS-THIRD-ARG during the attempt to prove

; ; a goal.

; ; First, we only want to open up GENERALIzE when we are looking at

; ; goals, not when we are simply asking about the witnessing

; ; substitution. I believe that this speeds up the proofs

; ; considerably.

; $; \ll 42 \gg$

(prove-lemma car-generalize (rewrite)

(equal (car (generalize sg state))

(cons (subst $t$ (invert $s g$ ) (caar state))

(cdar state))))

i; $443 \gg$

(disable generalize)

; i Inspection of the proof of a subgoal of MAIN-HYPS-RELIEVED-6-FIRST

; ; suggests that we need the following lemma. Actually, before the

; f final polishing it was the case that the following version sufficed.

;; But final polishing led me to prove a 'better' ' version, as well

$; ;$ as the lemma DISJOINT-SET-DIFF-GENERAL in "sets.events".
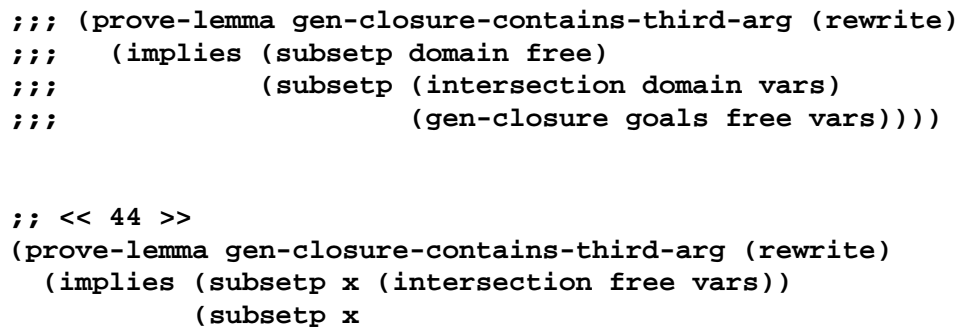

(gen-closure goals free vars))))

; $; \ll 45 \gg$

(prove-lemma main-hyps-relieved-6-first (rewrite)

(let ( (g (caar state))

(p (cdar state))

(free (cdr state))

(s (witnessing-instantiation (generalize sg state))))

(let ((new-g (subst $t$ (invert sg) g)))

(let ( (domain-1

(gen-closure (cons new-g p) free (all-vars $t$ new-g))))

(let ( (s1 (restrict $s$ domain-1))

(s2 (apply-to-subst (nullify-subst sg)

(implies (and (generalize-okp sg state)

$$
\text { (co-restrict s domain-1)))) }
$$

(valid-state (generalize sg state)))

$($ theorem (subst $t($ append s1 s2) new-g))))))))

; ; Now we embark on the final goal, MAIN-HYPS-RELIEVED-6-REST. The

; i idea is that one splits the witnessing substitution $s$ into two

; ; appropriate parts, $s 1$ and $\mathbf{s} 2$. These parts are the respective

; ; restriction and (approximately) co-restriction of the original

; ; witnessing substitution $s$ to some set that is ' 'closed' ' in the

; ; appropriate sense. Actually, the co-restriction is allowed to have

; ; a substitution applied to it, whose domain is disjoint from the

; ; variables occurring in goals ''outside', that closure. Below we

; ; give the lemmas and the proof of MAIN-HYPS-RELIEVED-6 from those

; ; lemmas. But first let us introduce the necessary notions.

; $; \ll 46 \gg$

(defn all-vars-disjoint-or-subsetp ( $p$ free $x$ )

; ; says that every goals's free variables are either contained

$;$; in $x$ or are disjoint from $x$ 


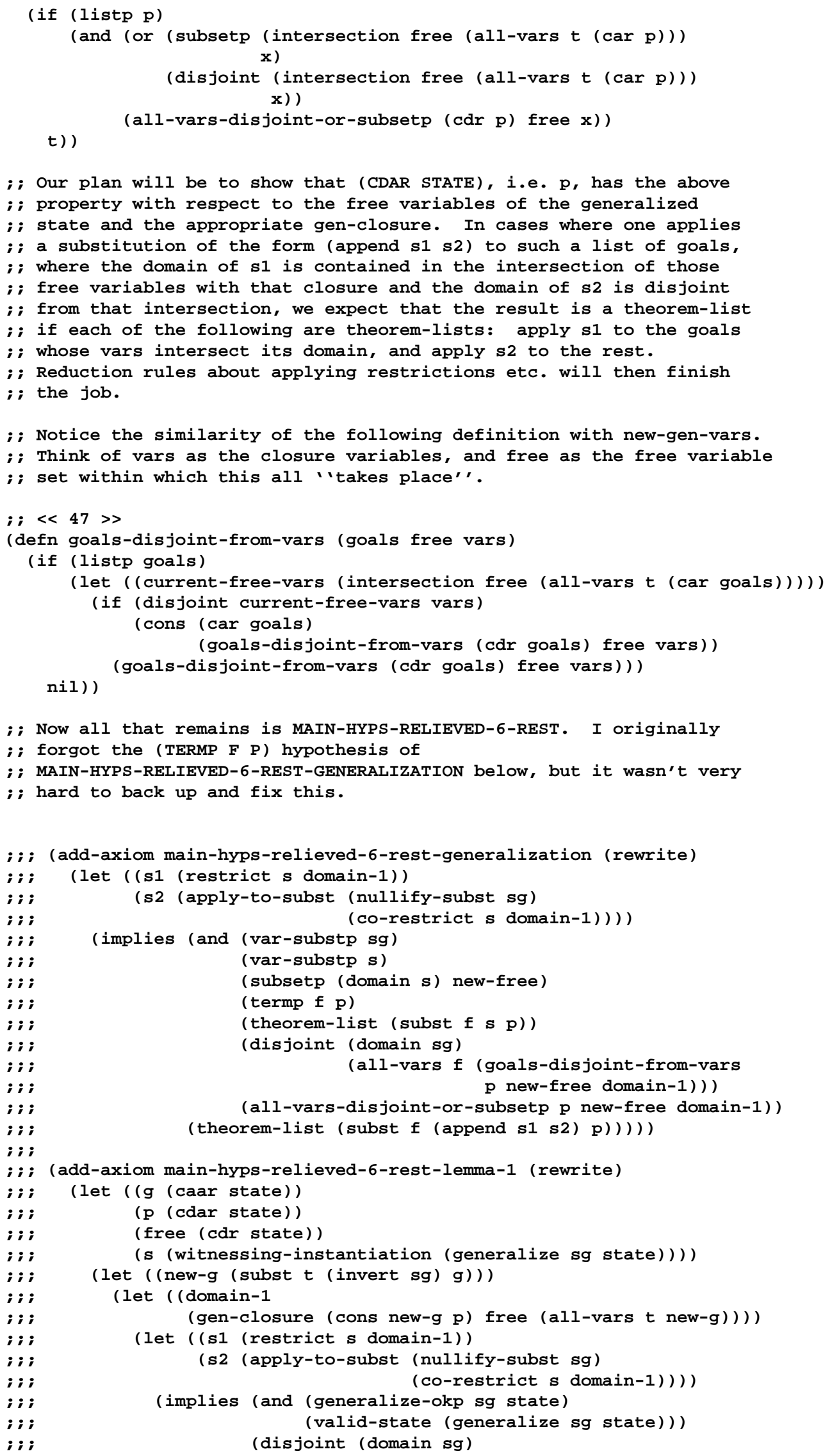




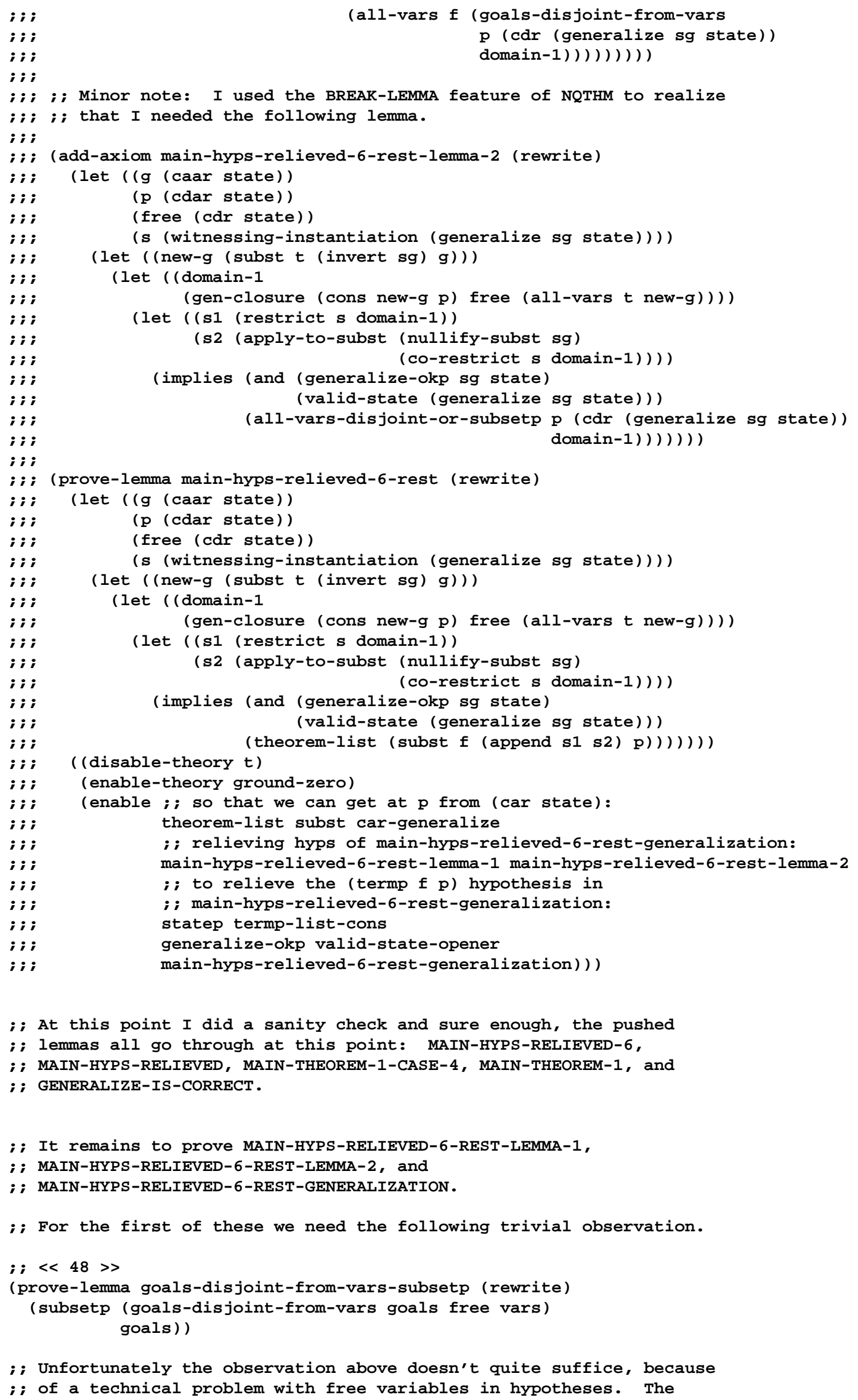




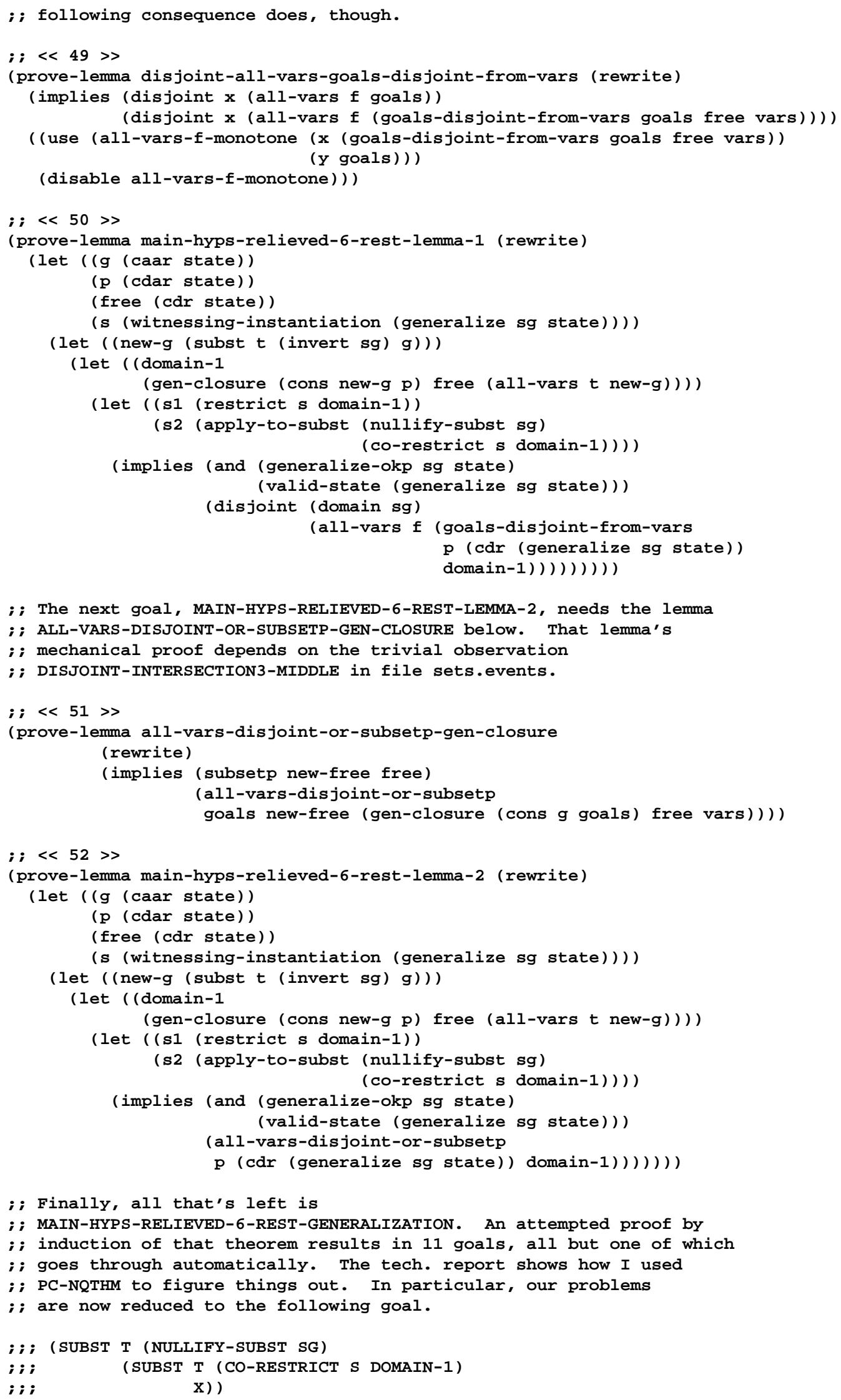




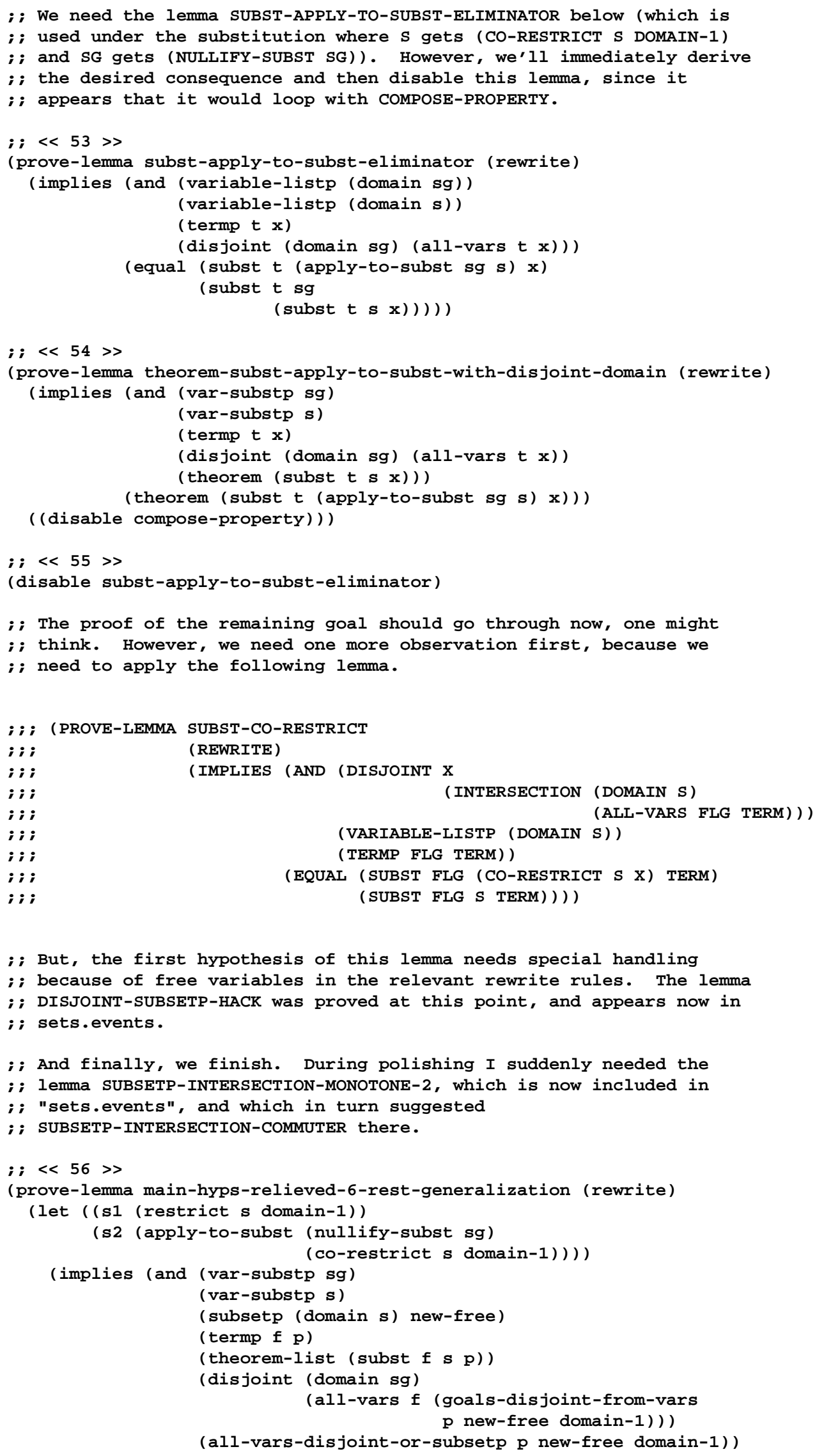


(theorem-list (subst $f($ append $s 1$ s2) p)))))

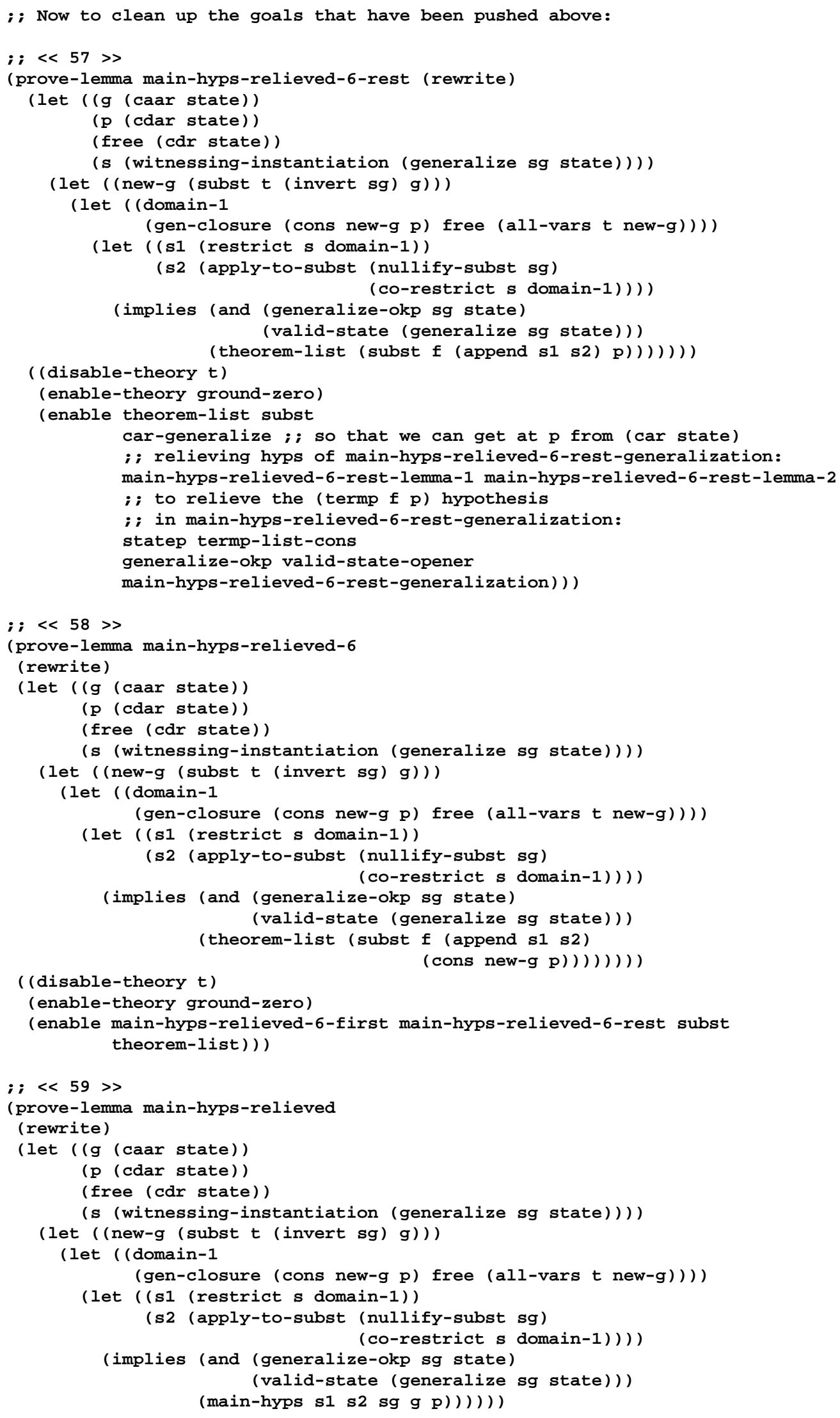




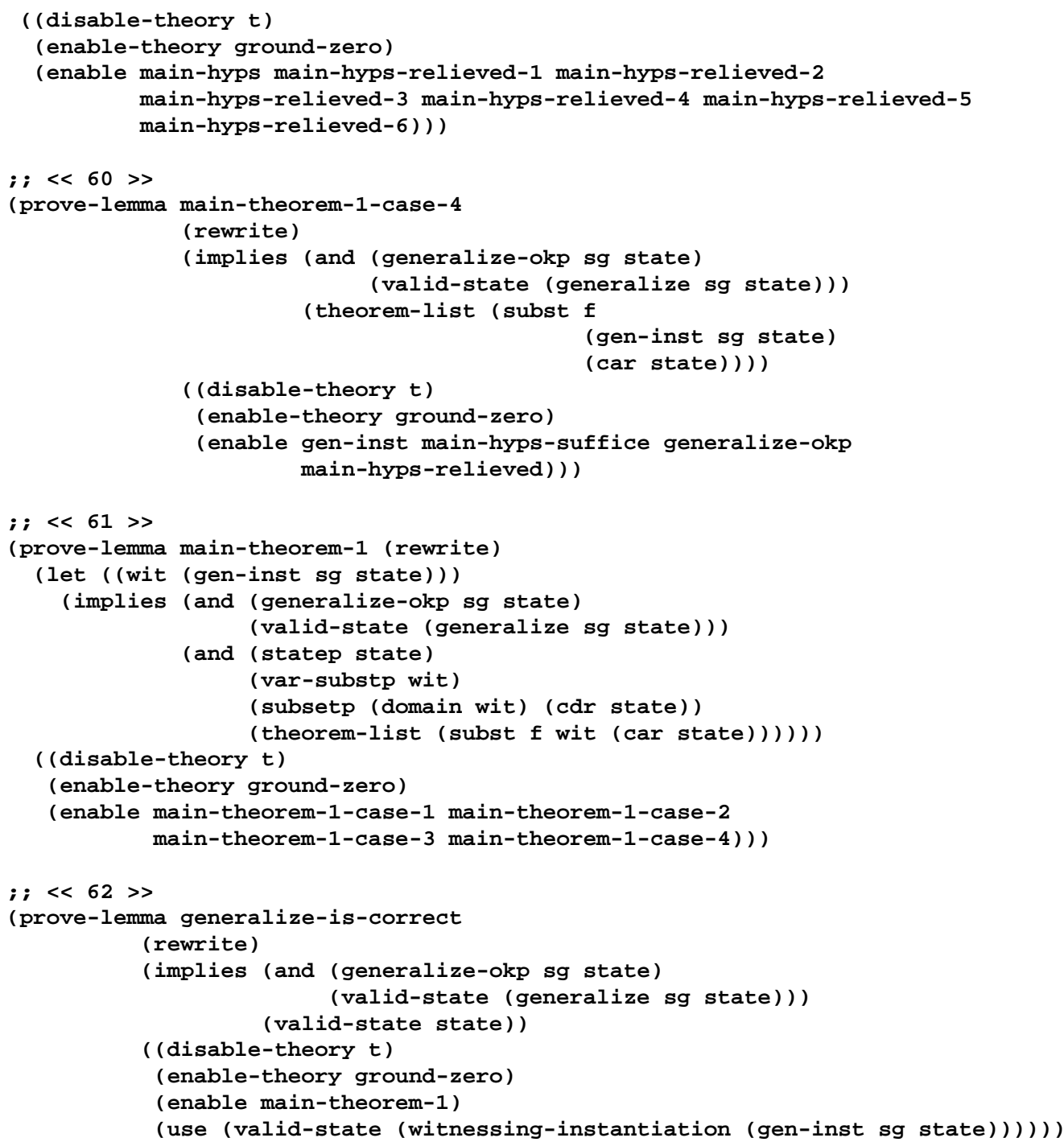

)) 


\section{References}

[1] Robert S. Boyer and J Strother Moore.

A Computational Logic.

Academic Press, New York, 1979.

[2] R.S. Boyer and J S. Moore.

Metafunctions: proving them correct and using them efficiently as new proof procedures.

The Correctness Problem in Computer Science.

Academic Press, 1981, pages 103-185.

[3] R. S. Boyer and J S. Moore.

A Computational Logic Handbook.

Academic Press, Boston, 1988.

[4] R. S. Boyer, D. M. Goldschlag, M. Kaufmann, and J S. Moore.

Functional Instantiation in First Order Logic, Report 44.

Technical Report, Computational Logic, 1717 W. 6th St., Austin, Texas, 78703, U.S.A., 1989.

To appear in the proceedings of the 1989 Workshop on Programming Logic, Programming Methodology Group, University of Goteborg.

[5] R.L. Constable, et al.

Implementing Mathematics with the Nuprl Proof Development System.

Prentice Hall, 1986.

[6] M. Davis and J. T. Schwartz.

Metamatehmatical extensibility for theorem verifiers and proof-checkers.

Computers and Mathematics with Applications 5:217-230, 1979.

[7] M. J. Gordon, A. J. Milner, and C. P. Wadsworth.

Edinburgh LCF.

Springer-Verlag, New York, 1979.

[8] M. Gordon.

HOL: A Proof Generating System for Higher-Order Logic.

Technical Report 103, University of Cambridge, Computer Laboratory, 1987.

[9] D. J. Howe.

Computational metatheory in Nuprl.

In 9th International Conference on Automated Deduction, pages 238-257. Springer-Verlag, 1988.

[10] Matt Kaufmann.

A user's manual for an interactive enhancement to the Boyer-Moore Theorem Prover.

Technical Report 19, Computational Logic, Inc., Austin, Texas, May, 1988.

[11] Matt Kaufmann.

Addition of free variables to an interactive enhancement of the Boyer-Moore Theorem Prover.

Technical Report 42, Computational Logic, Inc., Austin, Texas, May, 1989.

[12] Matt Kaufmann.

DEFN-SK: An extension of the Boyer-Moore Theorem Prover to handle first-order quantifiers.

Technical Report 43, Computational Logic, Inc., Austin, Texas, June, 1989.

[13] Todd B. Knoblock.

A formal metalanguage for NuPrl.

to appear.

[14] T. B. Knoblock and R. L. Constable.

Formalized metareasoning in type theory.

In Proceedings of the First Annual Symposium on Logic in Computer Science. IEEE, 1976.

[15] A. Quaife.

Automated proofs of Loeb's Theorem and Goedel's two imcompleteness theorems.

Journal of Automated Reasoning 4:219-231, 1988. 
[16] N. Shankar.

Towards Mechanical Metamathematics.

Journal of Automated Reasoning 1(1), 1985.

[17] Guy L. Steele Jr.

Common Lisp: The Language.

Digital Press, 1984.

[18] R. W. Weyhrauch.

Prolegomena to a theory of formal reasoning.

Artificial Intelligence 13:133-170, 1980. 


\section{Table of Contents}

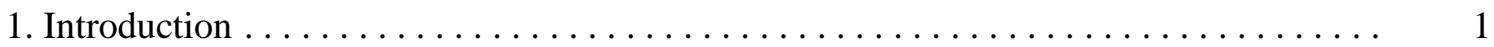

1.1. Introduction to the Boyer-Moore logic and theorem prover $\ldots \ldots \ldots \ldots \ldots \ldots \ldots \ldots \ldots 2$

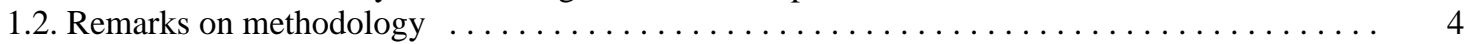

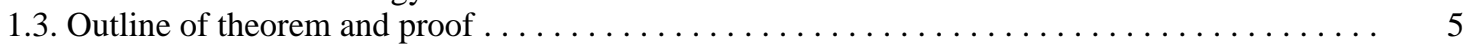

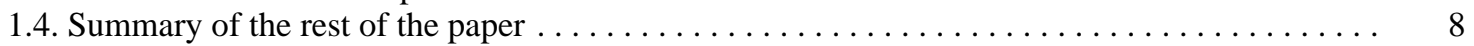

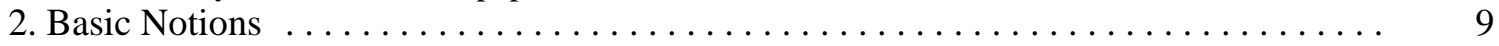

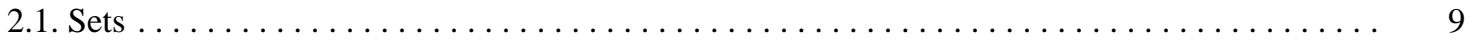

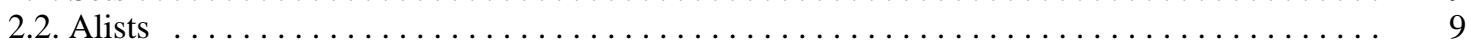

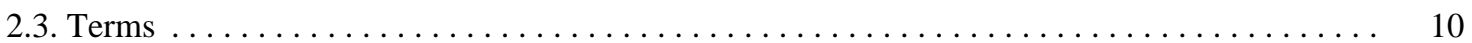

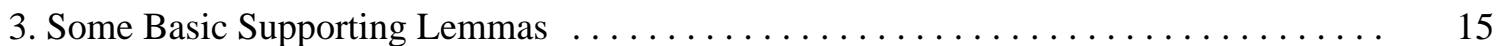

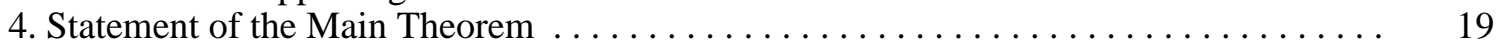

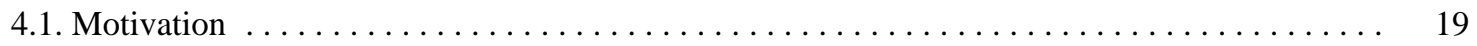

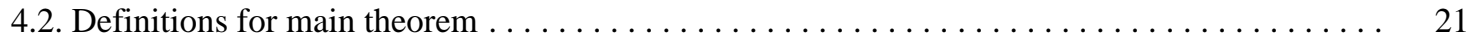

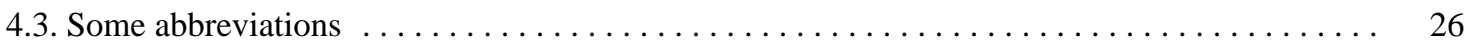

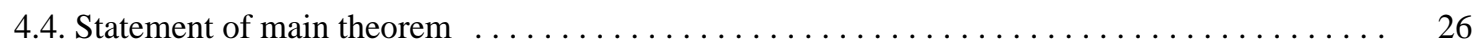

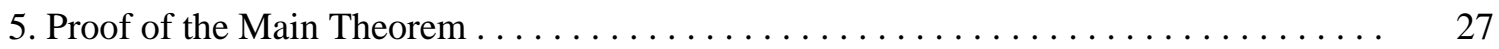

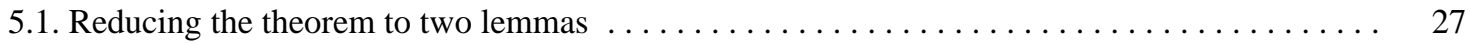

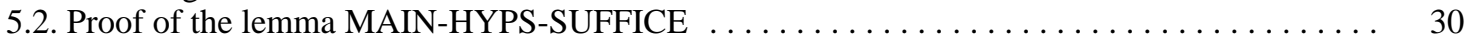

5.2.1. Proof of the lemma MAIN-HYPS-SUFFICE-FIRST $\ldots \ldots \ldots \ldots \ldots \ldots \ldots \ldots \ldots \ldots \ldots$

5.2.2. Proof of the lemma MAIN-HYPS-SUFFICE-REST $\ldots \ldots \ldots \ldots \ldots \ldots \ldots \ldots \ldots \ldots \ldots \ldots$

5.3. Proof of the lemma MAIN-HYPS-RELIEVED $\ldots \ldots \ldots \ldots \ldots \ldots \ldots \ldots \ldots \ldots \ldots \ldots \ldots \ldots \ldots \ldots$

5.3.1. Proof of the lemma MAIN-HYPS-RELIEVED-5 $\ldots \ldots \ldots \ldots \ldots \ldots \ldots \ldots \ldots \ldots \ldots \ldots \ldots$

5.3.2. Proof of the lemma MAIN-HYPS-RELIEVED-6 $\ldots \ldots \ldots \ldots \ldots \ldots \ldots \ldots \ldots \ldots \ldots \ldots$

5.3.2(1). Proof of the lemma MAIN-HYPS-RELIEVED-6-FIRST $\ldots \ldots \ldots \ldots \ldots \ldots \ldots . \quad 39$

5.3.2(2). Proof of the lemma MAIN-HYPS-RELIEVED-6-REST $\ldots \ldots \ldots \ldots \ldots \ldots \ldots .40$

5.3.2(3). Some comments on the proof of the lemma MAIN-HYPS-RELIEVED-6-REST-

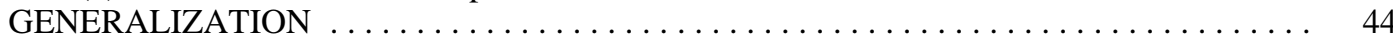

Appendix A. Events Files: sets, alists, terms, and generalize $\ldots \ldots \ldots \ldots \ldots \ldots \ldots .47$ 